\title{
Floer homology and splicing knot complements
}

\author{
EAMAN EFTEKHARY
}

We obtain a formula for the Heegaard Floer homology (hat theory) of the threemanifold $Y\left(K_{1}, K_{2}\right)$ obtained by splicing the complements of the knots $K_{i} \subset Y_{i}$, $i=1,2$, in terms of the knot Floer homology of $K_{1}$ and $K_{2}$. We also present a few applications. If $h_{n}^{i}$ denotes the rank of the Heegaard Floer group $\widehat{\mathrm{HFK}}$ for the knot obtained by $n$-surgery over $K_{i}$, we show that the rank of $\widehat{\mathrm{HF}}\left(Y\left(K_{1}, K_{2}\right)\right)$ is bounded below by

$$
\left|\left(h_{\infty}^{1}-h_{1}^{1}\right)\left(h_{\infty}^{2}-h_{1}^{2}\right)-\left(h_{0}^{1}-h_{1}^{1}\right)\left(h_{0}^{2}-h_{1}^{2}\right)\right| .
$$

We also show that if splicing the complement of a knot $K \subset Y$ with the trefoil complements gives a homology sphere $L$-space, then $K$ is trivial and $Y$ is a homology sphere $L$-space.

57M27; 57R58

\section{Introduction}

Heegaard Floer homology, introduced by Ozsváth and Szabó [12], has been the source of powerful techniques for the study of objects in low-dimensional topology. It is interesting to investigate whether Heegaard Floer homology can distinguish the standard sphere from other homology spheres. Since the Heegaard Floer groups of the connected sum of two homology spheres are obtained as the tensor product of the Heegaard Floer groups associated with the two pieces, the question is reduced to determining prime homology spheres with trivial Heegaard Floer groups. The Poincaré homology sphere $\Sigma(2,3,5)$ with either orientation is the unique known example of a non-trivial prime homology sphere $Y$ with $\widehat{\mathrm{HF}}(Y ; \mathbb{Z})=\mathbb{Z}$. A conjecture of Ozsváth and Szabó predicts that this is in fact the only possible example.

In this paper we study the Heegaard Floer groups of a homology sphere $Y$ which contains an incompressible torus. We may use the incompressible torus to decompose $Y$, fill out the torus boundary of each of the two pieces by gluing a solid torus, and obtain two new homology spheres, $Y_{1}$ and $Y_{2}$. By requiring $Y_{1}$ and $Y_{2}$ to be homology spheres the gluing of the solid tori is determined; the decomposition determines a knot $K_{i}$ in $Y_{i}, i=1,2$, and $Y=Y\left(K_{1}, K_{2}\right)$ is obtained by splicing the complements of 
$K_{1}$ and $K_{2}$ in $Y_{1}$ and $Y_{2}$, respectively. A formula is obtained for $\widehat{\mathrm{HF}}(Y ; \mathbb{F})$ in terms of the knot Floer objects associated with $K_{1} \subset Y_{1}$ and $K_{2} \subset Y_{2}$, where $\mathbb{F}$ denotes the field $\mathbb{Z} / 2 \mathbb{Z}$ with two elements.

The more precise statement of the splicing formula obtained in this paper is as follows. Let $K \subset Y$ denote a null-homologous knot inside a three-manifold $Y$. For every $n \in \mathbb{Z} \cup\{\infty\}$ let $Y_{n}=Y_{n}(K)$ denote the three-manifold obtained by performing $n$-surgery on $K$ and let $K_{n} \subset Y_{n}$ denote the knot in $Y_{n}$ which is the core of the neighbourhood replaced for nd $(K) \subset Y$ in constructing $Y_{n}$. Denote the homology group $\widehat{\mathrm{HFK}}\left(Y_{n}, K_{n} ; \mathbb{F}\right)$ by $\mathbb{H}_{n}(K)$ and its dimension as a vector space over $\mathbb{F}$ by $h_{n}(K)$. In particular, $\mathbb{H}_{\infty}(K)=\widehat{\operatorname{HFK}}(Y, K ; \mathbb{F})$ and $\mathbb{H}_{0}(K)=\widehat{\operatorname{HFL}}(Y, K ; \mathbb{F})$ are the knot Floer homology and the longitude Floer homology of $K$, respectively (see Ozsváth and Szabó [11] and Eftekhary [2]).

Choose a Heegaard diagram

$$
H=\left(\Sigma, \boldsymbol{\alpha}=\left\{\alpha_{1}, \ldots, \alpha_{g}\right\}, \hat{\boldsymbol{\beta}}=\left\{\beta_{1}, \ldots, \beta_{g-1}\right\}\right)
$$

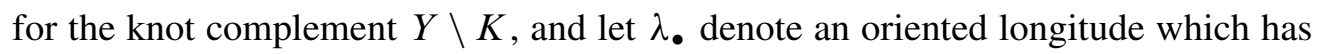
framing coefficient $\bullet \in \mathbb{Z} \cup\{\infty\}$. One can choose the curves $\lambda_{\bullet}$ (which are disjoint from the curves in $\widehat{\boldsymbol{\beta}})$ so that the pairs $\left(\lambda_{0}, \lambda_{\infty}\right),\left(\lambda_{1}, \lambda_{\infty}\right)$ and $\left(\lambda_{0}, \lambda_{1}\right)$ have single intersection points in the Heegaard diagram. For $\bullet \in\{0,1, \infty\}$ set

$$
\boldsymbol{\beta}_{\bullet}=\left\{\beta_{1}^{\bullet}, \ldots, \beta_{g-1}^{\bullet}, \lambda_{\bullet}\right\},
$$

where $\beta_{i}^{\bullet}$ is an isotopic copy of the curve $\beta_{i}$. The pictures on the left-hand side and the right-hand side of Figure 1 illustrate two possible general arrangements for the curves $\lambda_{0}, \lambda_{1}$ and $\lambda_{\infty}$. In Figure 1 and other figures in this paper, the surface orientation is chosen opposite from the standard orientation of the page in order to stay compatible with the orientation convention of [12].

The two Heegaard quadruples

$$
\left(\Sigma, \boldsymbol{\alpha}, \boldsymbol{\beta}_{0}, \boldsymbol{\beta}_{1}, \boldsymbol{\beta}_{\infty} ; u, v, w\right) \quad \text { and } \quad\left(\Sigma, \boldsymbol{\alpha}, \boldsymbol{\beta}_{0}, \boldsymbol{\beta}_{1}, \boldsymbol{\beta}_{\infty} ; \bar{u}, \bar{v}, \bar{w}\right)
$$

obtained in this way then correspond to the exact triangles

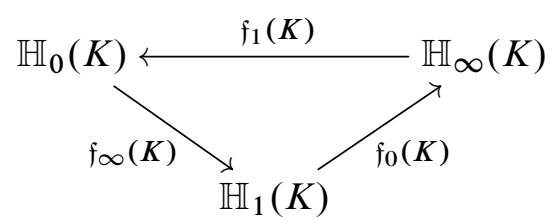

and

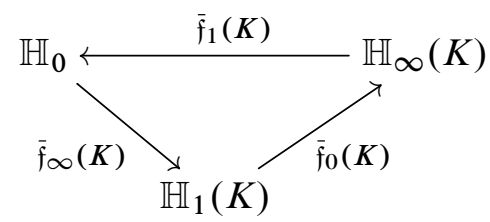



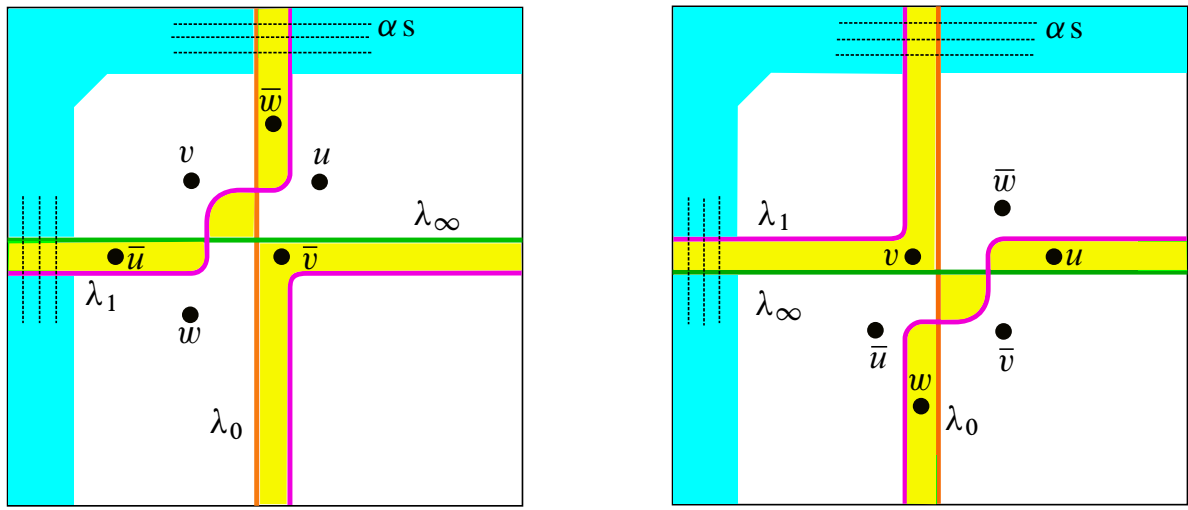

Figure 1: The curves $\lambda_{0}$ (orange), $\lambda_{1}$ (pink) and $\lambda_{\infty}$ (green) and the punctures are chosen following one of the above two patterns. The punctures $u, v$ and $w$ are used to define $\mathfrak{f}_{0}, \mathfrak{f}_{1}$ and $\mathfrak{f}_{\infty}$, while $\bar{u}, \bar{v}$ and $\bar{w}$ are used to define $\overline{\mathfrak{f}}_{0}, \overline{\mathfrak{f}}_{1}$ and $\overline{\mathfrak{f}}_{\infty}$.

respectively. The ranks of both $\mathfrak{f}_{\bullet}(K)$ and $\overline{\mathfrak{f}}_{\bullet}(K)$ are equal to

$$
a_{\bullet}(K)=\frac{1}{2}\left(h_{0}(K)+h_{1}(K)+h_{\infty}(K)-2 h_{\bullet}(K)\right), \quad \bullet \in\{0,1, \infty\} .
$$

The exactness of the above two triangles imply that the induced maps

$$
\operatorname{Coker}\left(\mathfrak{f}_{0}(K)\right) \longrightarrow \operatorname{Ker}\left(\mathfrak{f}_{\infty}(K)\right) \text { and } \operatorname{Coker}\left(\overline{\mathfrak{f}}_{0}(K)\right) \longrightarrow \operatorname{Ker}\left(\overline{\mathfrak{f}}_{\infty}(K)\right)
$$

by $\mathfrak{f}_{1}(K)$ and $\overline{\mathfrak{f}}_{1}(K)$ are isomorphisms. Both the domain and the target of the aforementioned isomorphisms are of dimension $a_{1}(K)$. Take $\theta(K): \mathbb{H}_{0}(K) \rightarrow \mathbb{H}_{\infty}(K)$ (resp. $\left.\bar{\theta}(K): \mathbb{H}_{0}(K) \rightarrow \mathbb{H}_{\infty}(K)\right)$ to be an arbitrary extension of the inverse of the isomorphism induced by $\mathfrak{f}_{1}(K)$ (resp. $\left.\overline{\mathfrak{f}}_{1}(K)\right)$, so that the ranks of both $\theta(K)$ and $\bar{\theta}(K)$ are equal to $a_{1}(K)$.

Suppose that a pair of knots $K_{1}$ and $K_{2}$ is given. For every $\star, \bullet \in\{0,1, \infty\}$ and $i=1,2$, set $\mathbb{H}_{\bullet}^{i}=\mathbb{H}_{\bullet}\left(K_{i}\right), \mathbb{H}_{\star, \bullet}=\mathbb{H}_{\star}^{1} \otimes \mathbb{H}_{\bullet}^{2}, \mathfrak{f}_{\bullet}^{i}=\mathfrak{f}_{\bullet}\left(K_{i}\right), \overline{\mathfrak{f}}_{\bullet}^{i}=\overline{\mathfrak{f}}_{\bullet}\left(K_{i}\right), \theta^{i}=\theta\left(K_{i}\right)$ and $\bar{\theta}^{i}=\bar{\theta}\left(K_{i}\right)$. Consider the chain complex (四 $\left.\left(K_{1}, K_{2}\right), d_{\text {国 }}\right)$ constructed as follows.

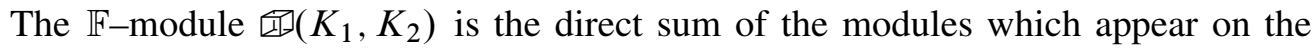
vertices of the cube illustrated in Figure 2.

Each directed edge (including the dashed edges) in the aforementioned diagram determines a homomorphism from $⿴ 囗 ⿱ 一 一)\left(K_{1}, K_{2}\right)$ to itself, which is trivial on all summands except for the one which corresponds to its start point. The map takes the summand corresponding to its start point to the summand corresponding to its endpoint by the homomorphism which labels the directed edge. The differential $d_{\text {四 }}$ of the complex 四 $\left(K_{1}, K_{2}\right)$ is defined to be the sum of the homomorphisms which correspond to the 


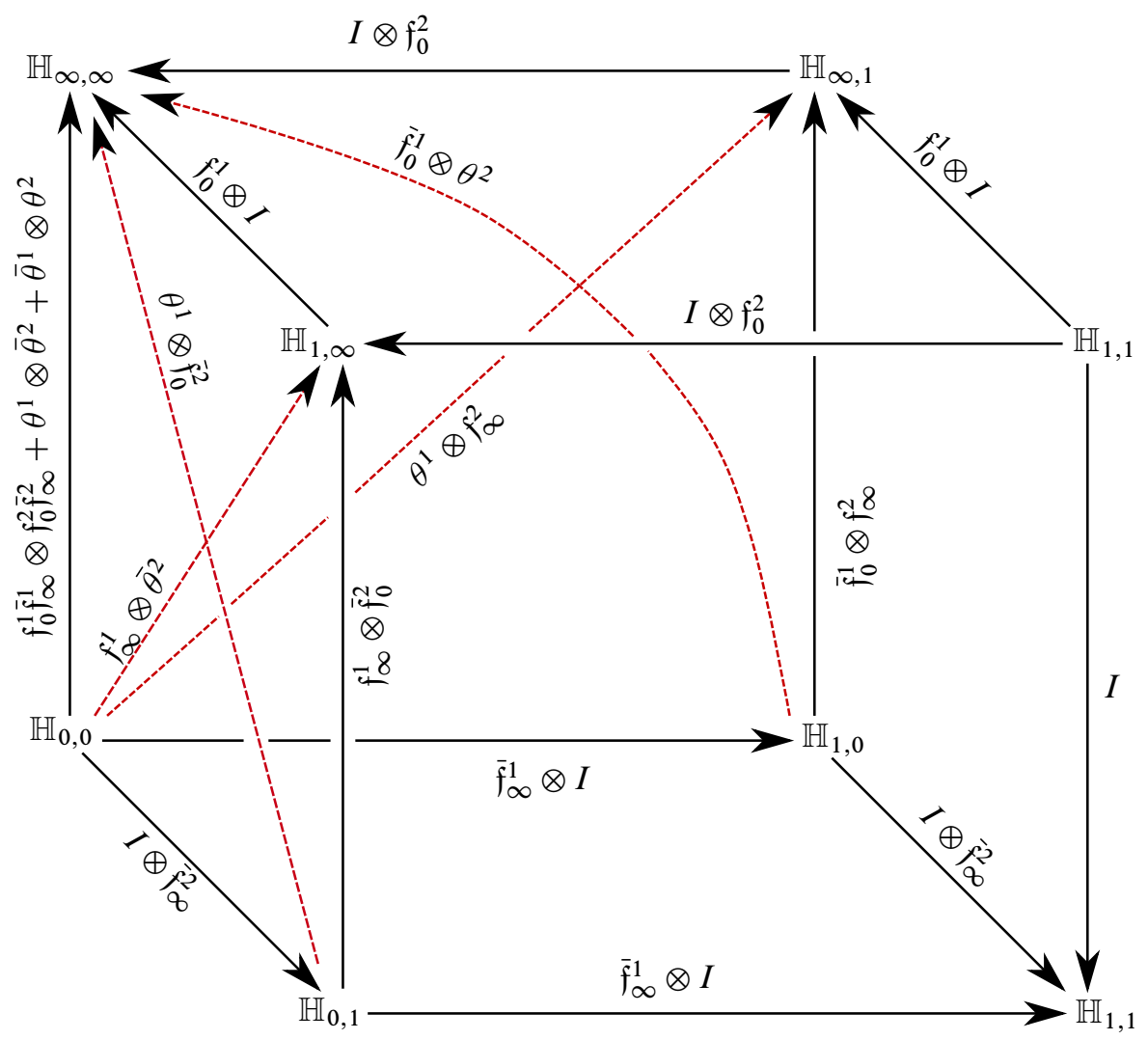

Figure 2: The above cube determines the chain complex 四 $\left(K_{1}, K_{2}\right)$ and its differential $d_{\text {田 }}$

directed edges of the cube in Figure 2. One should of course make sure that $d_{\text {国 }} \circ d_{\text {国 }}=0$. However, this follows quickly from the exactness of the triangle in (1).

Theorem 1.1 With the above notation fixed, the Heegaard Floer homology of the three-manifold $Y\left(K_{1}, K_{2}\right)$ obtained by splicing the knot complements $Y_{1} \backslash K_{1}$ and $Y_{2} \backslash K_{2}$ is given by

$$
\widehat{\mathrm{HF}}\left(Y\left(K_{1}, K_{2}\right) ; \mathbb{F}\right) \simeq H_{*}\left(\text { 四 }\left(K_{1}, K_{2}\right), d_{\text {四 }}\right) .
$$

We use the combinatorial description of Heegaard Floer homology by Sarkar and Wang [16], which is also adapted for knots in $S^{3}$ by Manolescu, Ozsváth and Sarkar [8] and Manolescu, Ozsváth, Szabó and Thurston [9]. These combinatorial descriptions help us avoid several technical issues that arise when one glues holomorphic curves. 
For the knots $K_{1} \subset Y_{1}$ and $K_{2} \subset Y_{2}$ as above, define

$$
\chi\left(K_{1}, K_{2}\right):=\left(h_{\infty}^{1}-h_{1}^{1}\right)\left(h_{\infty}^{2}-h_{1}^{2}\right)-\left(h_{0}^{1}-h_{1}^{1}\right)\left(h_{0}^{2}-h_{1}^{2}\right) .
$$

As a corollary of Theorem 1.1 we prove the following:

Corollary 1.2 For $Y=Y\left(K_{1}, K_{2}\right)$ as above we have

$$
\operatorname{rnk}(\widehat{\mathrm{HF}}(Y ; \mathbb{F})) \geq \max \left\{\left|\chi\left(K_{1}, K_{2}\right)\right|,\left|\chi\left(\overline{K_{1}}, \overline{K_{2}}\right)\right|\right\},
$$

where $\bar{K}_{i} \subset \bar{Y}_{i}=-Y_{i}$ denotes the mirror of $K_{i}$ in the three-manifold $\bar{Y}_{i}=-Y_{i}$ for $i=1,2$.

When one of the two knots is the trefoil, the formula is simplified significantly. In particular, we prove the following corollary in Section 6:

Corollary 1.3 Let $R$ denote the right-handed trefoil. With the above notation fixed, $\operatorname{rnk}(\widehat{\mathrm{HF}}(Y(R, K))) \leq h_{0}(K)+h_{1}(K)$, $\operatorname{rnk}(\widehat{\mathrm{HF}}(Y(R, K))) \geq 4 \max \left\{h_{0}(K), h_{1}(K), h_{\infty}(K)\right\}-\left(h_{0}(K)+h_{1}(K)+2 h_{\infty}(K)\right)$. Moreover, if $K$ is non-trivial, $Y(R, K)$ is not an $L$-space.

It is shown by Hedden and Levine [5] that splicing non-trivial knots inside homology sphere $L$-spaces never produces an $L$-space. Meanwhile, the knot $K$ in Corollary 1.3 lives in an arbitrary homology sphere. In this regard, Corollary 1.3 goes beyond the result of Hedden and Levine.

Remark 1.4 The splicing formula of Theorem 1.1 is different from the splicing formula from the original arXiv version of the paper. The results of a few other papers of the author are based on the splicing formula of this paper. The results of [3] remain unchanged, since the formula (17) presented in Section 5.1 which is used in [3] remains unchanged. The proof of the main theorem of [4] no longer goes through. Fixing the argument requires developing some technology, including a description of the bordered Floer homology for a knot complement only in terms of the knot chain complex associated with the knot. The modifications will appear in an upcoming revision of [4].

\section{Acknowledgements}

The author would like to thank Zoltán Szabó for his suggestions. In particular, it was his idea that the difficulties with gluing could disappear if the combinatorial approach of Sarkar and Wang [16] were used. He would also like to thank Matt Hedden and Adam Levine, as well as the anonymous referee for pointing out (several) mistakes in the earlier versions of this paper. 


\section{Graphs of chain complexes}

\subsection{Oriented graphs and chain complexes}

Let $G$ denote an oriented graph without oriented loops, which consists of a set $V(G)$ of vertices and a set

$$
E(G) \subset V(G) \times V(G)
$$

of directed edges. For every $e=\left(v_{1}, v_{2}\right) \in E(G)$ we let $v_{s}(e)=v_{2}$ and $v_{t}(e)=v_{1}$. The edge $e$ is thus oriented from its starting vertex $v_{s}(e)$ towards its terminal vertex $v_{t}(e)$. The condition that $G$ does not contain any oriented loops implies that there is no sequence $e_{1}, \ldots, e_{k} \in E(G)$ with the property

$$
v_{t}\left(e_{i}\right)=v_{s}\left(e_{i+1}\right), \quad i=1, \ldots, k-1, \quad \text { and } \quad v_{t}\left(e_{k}\right)=v_{s}\left(e_{1}\right) .
$$

Definition 2.1 Let $G$ denote an oriented graph without any oriented loops, as above. A collection $\left\{\left(C_{v}, d_{v}\right)\right\}_{v \in V(G)}$ of chain complexes, together with the chain maps

$$
\left\{f_{e}: C_{v_{s}(e)} \rightarrow C_{v_{t}(e)} \mid e \in E(G)\right\}
$$

is called a graph of complexes if, for every $v_{1}, v_{2} \in V(G)$,

$$
\sum_{\substack{e_{1}, e_{2} \in E(G) \\ v_{s}\left(e_{1}\right)=v_{1}, v_{t}\left(e_{2}\right)=v_{2} \\ v_{t}\left(e_{1}\right)=v_{s}\left(e_{2}\right)}} f_{e_{2}} \circ f_{e_{1}}=0 .
$$

Associated with a graph of complexes as above, write $C_{G}=\bigoplus_{v \in V(G)} C_{v}$ and define the differential $d_{G}: C_{G} \rightarrow C_{G}$ as follows. For $c \in C_{v} \subset C_{G}$, let

$$
d_{G}(c)=\sum_{w \in V(G)} d_{G, w}(c)
$$

where $d_{G, w}(c) \in C_{w}$ is defined by

$$
d_{G, w}(c)= \begin{cases}d_{v}(c) & \text { if } w=v \\ f_{e}(c) & \text { if there exists } e \in E(G) \text { with } v_{s}(e)=v \text { and } v_{t}(e)=w \\ 0 & \text { otherwise. }\end{cases}
$$

Definition 2.2 The chain complex $\left(C_{G}, d_{G}\right)$ is called the chain complex associated with the graph $G$ of chain complexes.

The condition (2) implies that $d_{G} \circ d_{G}=0$, ie that $\left(C_{G}, d_{G}\right)$ is a chain complex, since each $f_{e}$ is a chain map. The chain complex $\left(C_{G}, d_{G}\right)$ is usually represented by drawing 
the oriented graph $G$, labelling each vertex $v \in V(G)$ by the chain complex $\left(C_{v}, d_{v}\right)$ (or simply by $C_{v}$ if there is no confusion) and labelling each oriented edge $e$ by the chain map $f_{e}$.

Let $G$ denote an oriented graph without any loops. It is then possible to label the vertices of $G$ by $1,2, \ldots, n$ so that for each $e \in E(G)$ we have $v_{s}(e)<v_{t}(e)$ (as numbers in $\{1, \ldots, n\})$. Correspondingly, the chain complexes associated with the vertices of $G$ may be labelled $\left(C_{1}, d_{1}\right), \ldots,\left(C_{n}, d_{n}\right)$. Let $H$ denote the graph with vertices $1, \ldots, n$ and edges

$$
E(H)=\{(i, j) \mid i, j \in\{1, \ldots, n\} \text { and } i>j\} .
$$

For $e \in E(H)$ let $g_{e}=f_{e}$ if $e \in E(G)$ and $g_{e}=0$ otherwise. Associated with $\left\{C_{i}\right\}_{i \in V(H)}$ and $\left\{g_{e}\right\}_{e \in E(H)}$ we thus find the complex $\left(C_{H}, d_{H}\right)$, which is identified with $\left(C_{G}, d_{G}\right)$. In other words, we may always assume that the underlying graph in a graph of complexes is the complete oriented graph $H$. The condition (2) in this case is equivalent to

$$
\sum_{i>k>j} g_{(i, k)} \circ g_{(k, j)}=0 \quad \text { for all } i, j \in\{1, \ldots, n\}
$$

\subsection{Replacing chain complexes with their homology}

When the ring of coefficients is $\mathbb{F}=\mathbb{Z} / 2 \mathbb{Z}$ we would like to replace each complex $\left(C_{i}, d_{i}\right)$ in $\left(C_{H}, d_{H}\right)$ with $\left(H_{*}\left(C_{i}, d_{i}\right), 0\right)$, at the expense of modifying the chain maps $\left\{g_{e}\right\}_{e \in E(H)}$ so that the homology of the chain complex associated with the graph of chain complexes remains intact. Let us begin with a lemma.

Lemma 2.3 Suppose that a chain complex $\left(C, d_{C}\right)$ is decomposed, as a vector space over $\mathbb{F}$, as $C \simeq A \oplus A \oplus B$ for some vector spaces $A$ and $B$. Suppose that the differential $d_{C}$ of $C$ has the following block form in this decomposition:

$$
d_{C}=\left(\begin{array}{ccc}
0 & I_{A} & f_{1} \\
0 & 0 & f_{2} \\
g_{1} & g_{2} & h
\end{array}\right)
$$

Then $d_{B}=h+g_{2} f_{1}: B \rightarrow B$ is a differential and $H_{*}\left(C, d_{C}\right)=H_{*}\left(B, d_{B}\right)$.

Proof Since $d_{C}$ is a differential, $f_{1} g_{2}=0$ and the matrix

$$
P=\left(\begin{array}{ccc}
I & 0 & 0 \\
0 & I & f_{1} \\
g_{2} & 0 & I
\end{array}\right)
$$


is thus its own inverse. Since $d_{C}^{2}=0$ we get

$$
P\left(\begin{array}{ccc}
0 & I_{A} & f_{1} \\
0 & 0 & f_{2} \\
g_{1} & g_{2} & h
\end{array}\right) P=\left(\begin{array}{ccc}
0 & I_{A} & 0 \\
0 & 0 & 0 \\
0 & 0 & h+g_{2} f_{1}
\end{array}\right) .
$$

This completes the proof of the lemma.

We refer to the procedure which changes the chain complex $\left(C, d_{C}\right)$ to the chain complex $\left(B, d_{B}\right)$ as the cancellation of the two subspaces $A \oplus 0 \oplus 0 \simeq A$ and $0 \oplus A \oplus 0 \simeq A$ of $C$ against each other.

The differential $d_{i}$ of $C_{i}$ may be used to decompose $C_{i}$ as $A_{i}^{1} \oplus H_{i} \oplus A_{i}^{2}$, where $A_{i}^{1}$ and $A_{i}^{2}$ are two copies of the same $\mathbb{F}$-module $A_{i}$, so that $d_{i}$ takes the form

$$
d_{i}=\left(\begin{array}{ccc}
0 & 0 & I_{A_{i}} \\
0 & 0 & 0 \\
0 & 0 & 0
\end{array}\right), \quad i=1, \ldots, n .
$$

Note that $H_{i}=H_{*}\left(C_{i}, d_{i}\right)$ is in fact the homology of the complex $C_{i}$. In particular, $H_{i} \subset \operatorname{Ker}\left(d_{i}: C_{i} \rightarrow C_{i}\right)$. Since $d_{v_{t}(e)} \circ g_{e}=g_{e} \circ d_{v_{s}(e)}$, in this basis the matrix block presentation of $g_{e}$ is of the form

$$
g_{e}=\left(\begin{array}{ccc}
M_{e} & P_{e} & N_{e} \\
0 & G_{e} & Q_{e} \\
0 & 0 & M_{e}
\end{array}\right) \quad \text { for all } e \in E(H)
$$

Initially, the block presentation for $d_{H}$ is of the form

$$
d_{H}=\left(\begin{array}{ccccc}
d_{1} & 0 & 0 & \ldots & 0 \\
g_{(2,1)} & d_{2} & 0 & \ldots & 0 \\
g_{(3,1)} & g_{(3,2)} & d_{3} & \ldots & 0 \\
\vdots & \vdots & \vdots & \ddots & \vdots \\
g_{(n, 1)} & g_{(n, 2)} & g_{(n, 3)} & \ldots & d_{n}
\end{array}\right) .
$$

Replacing the above $3 \times 3$ block presentations for $g_{(i, j)}$ and $d_{i}$, the homomorphism $d_{H}$ takes a $3 n \times 3 n$ block presentation, where $n$ of the block entries are the identity matrices corresponding to $d_{1}, \ldots, d_{n}$. Lemma 2.3 may be used inductively to cancel $A_{i}^{1}$ against $A_{i}^{2}$ for $i=1, \ldots, n$ and modify the remaining blocks correspondingly. Straightforward linear algebra implies the following lemma:

Lemma 2.4 Fix the above notation and for $i, j \in\{1, \ldots, n\}$ let

$$
h_{(i, j)}=G_{(i, j)}+\sum_{\ell \geq 1} \sum_{i>k_{1}>k_{2}>\cdots>k_{\ell}>j} Q_{\left(i, k_{1}\right)} N_{\left(k_{1}, k_{2}\right)} \cdots N_{\left(k_{\ell-1}, k_{\ell}\right)} P_{\left(k_{\ell}, j\right)} .
$$


Then the homology of the chain complex associated with $H$ that has complexes $\left\{\left(C_{i}, d_{i}\right)\right\}_{i=1}^{n}$ and chain maps $\left\{g_{e}\right\}_{e \in E(H)}$ is isomorphic to the homology of the chain complex associated with $H$ that has complexes $\left\{\left(H_{i}, 0\right)\right\}_{i=1}^{n}$ and homomorphisms $\left\{h_{e}\right\}_{e \in E(H)}$.

For $\ell=1$ set $k=k_{1}$. For $h_{j} \in \operatorname{Ker}\left(G_{(k, j)}: H_{j} \rightarrow H_{k}\right), P_{(k, j)}\left(h_{j}\right)=d_{k}\left(a_{k}\right)$ for some $a_{k} \in A_{k}$. The element $a_{k}$ may of course be modified by adding to $a_{k}$ an element $h_{k} \in H_{k}$. From here, $Q_{(i, k)} P_{(k, j)}\left(h_{j}\right)$ is equal to $g_{(i, k)}\left(a_{k}\right)$ up to the addition of an element in $g_{(i, k)}\left(H_{k}\right)$. In particular, we find a natural well-defined map

$$
\theta_{(i>k>j)}: \operatorname{Ker}\left(G_{(k, j)}\right) \longrightarrow \operatorname{Coker}\left(G_{(i, k)}\right)
$$

and $Q_{(i, k)} P_{(k, j)}$ is an extension of $\theta_{(i>k>j)}$ to a homomorphism from $H_{j}$ to $H_{k}$. It is however important to note that simultaneous replacement of the maps $Q_{(i, k)} P_{(k, j)}$ with arbitrary extensions of $\theta(i>k>j)$ in Lemma 2.3 is not a priori possible.

In this paper, we will face situations where each complex $C_{i}$ is of the form $C_{i}^{1} \otimes C_{i}^{2}$ and each chain map $g_{(i, j)}: C_{j} \rightarrow C_{i}$ is of the form $g_{(i, j)}^{1} \otimes g_{(i, j)}^{2}$, where $g_{(i, j)}^{1}: C_{j}^{1} \rightarrow C_{i}^{1}$ and $g_{(i, j)}^{2}: C_{j}^{2} \rightarrow C_{i}^{2}$ are chain maps. In this situation, we may choose the decompositions $C_{i}^{r}=A_{i}^{r} \oplus H_{i}^{r} \oplus A_{i}^{r}$ for $r=1,2$. Subsequently, note that

$$
H_{i}=H_{i}^{1} \otimes H_{i}^{2} \quad \text { and } \quad A_{i}=\left(A_{i}^{1} \otimes A_{i}^{2}\right) \oplus\left(A_{i}^{1} \otimes H_{i}^{2}\right) \oplus\left(H_{i}^{1} \otimes A_{i}^{2}\right) \oplus\left(A_{i}^{1} \otimes A_{i}^{2}\right) .
$$

Moreover, corresponding to $g_{(i, j)}^{r}$ we obtain the blocks $M_{(i, j)}^{r}, N_{(i, j)}^{r}, P_{(i, j)}^{r}, Q_{(i, j)}^{r}$ and $G_{(i, j)}^{r}$ for $r=1,2$.

We close this section with a pair of simple lemmas addressing this situation.

Lemma 2.5 In the situation above,

$$
\begin{aligned}
Q_{(i, k)} P_{(k, j)}=Q_{(i, k)}^{1} P_{(k, j)}^{1} \otimes Q_{(i, k)}^{2} P_{(k, j)}^{2}+Q_{(i, k)}^{1} P_{(k, j)}^{1} \otimes G_{(i, k)}^{2} G_{(k, j)}^{2} & \\
& +G_{(i, k)}^{1} G_{(k, j)}^{1} \otimes Q_{(i, k)}^{2} P_{(k, j)}^{2} .
\end{aligned}
$$

Proof Choose $h_{j} \in H_{j}=H_{j}^{1} \otimes H_{j}^{2}$. The image of $h_{j}$ under $g_{(k, j)}$ is in

$$
\left(A_{k}^{1} \oplus H_{k}^{1} \oplus 0\right) \otimes\left(A_{k}^{2} \oplus H_{k}^{2} \oplus 0\right) \subset C_{k} .
$$

In particular, we find

$$
P_{(k, j)}\left(h_{j}\right) \in\left(A_{k}^{1} \otimes A_{k}^{2}\right) \oplus\left(A_{k}^{1} \otimes H_{k}^{2}\right) \oplus\left(H_{k}^{1} \otimes A_{k}^{2}\right) \oplus 0 \subset A_{k} .
$$

For $h_{j}=h_{j}^{1} \otimes h_{j}^{2}$ the corresponding decomposition of $P_{(k, j)}\left(h_{j}\right)$ is of the form

$$
\left(P_{(k, j)}^{1}\left(h_{j}^{1}\right) \otimes P_{(k, j)}^{2}\left(h_{j}^{2}\right), P_{(k, j)}^{1}\left(h_{j}^{1}\right) \otimes G_{(k, j)}^{2}\left(h_{j}^{2}\right), G_{(k, j)}^{1}\left(h_{j}^{1}\right) \otimes P_{(k, j)}^{2}\left(h_{j}^{2}\right), 0\right) .
$$


Note that $Q_{(i, k)} P_{(k, j)}\left(h_{j}\right)$ is the $H_{j}=H_{j}^{1} \otimes H_{j}^{2}$-component of $g_{(i, k)} P_{(k, j)}\left(h_{j}\right)$. The components in the above presentation are thus mapped by $Q_{(i, k)} P_{(k, j)}$ to

$$
\begin{gathered}
Q_{(i, k)}^{1} P_{(k, j)}^{1}\left(h_{j}^{1}\right) \otimes Q_{(i, k)}^{2} P_{(k, j)}^{2}\left(h_{j}^{2}\right), \quad Q_{(i, k)}^{1} P_{(k, j)}^{1}\left(h_{j}^{1}\right) \otimes G_{(i, k)}^{2} G_{(k, j)}^{2}\left(h_{j}^{2}\right), \\
G_{(i, k)}^{1} G_{(k, j)}^{1}\left(h_{j}^{1}\right) \otimes Q_{(i, k)}^{2} P_{(k, j)}^{2}\left(h_{j}^{2}\right) \quad \text { and } 0,
\end{gathered}
$$

respectively. This completes the proof of the lemma.

An interesting particular case of the above lemma is when one of the chain maps $g_{(i, k)}^{1}$ or $g_{(k, j)}^{1}$ is the identity, where we find

$$
\begin{aligned}
& g_{(i, k)}^{1}=\mathrm{Id} \Longrightarrow Q_{(i, k)} P_{(k, j)} G_{(k, j)}^{1} \otimes Q_{(i, k)}^{2} P_{(k, j)}^{2}, \\
& g_{(k, j)}^{1}=\mathrm{Id} \Longrightarrow Q_{(i, k)} P_{(k, j)}=G_{(i, k)}^{1} \otimes Q_{(i, k)}^{2} P_{(k, j)}^{2},
\end{aligned}
$$

respectively.

Lemma 2.6 With the above notation fixed, if $i>k>l>j$ and $g_{(i, k)}^{1}$ and $g_{(l, j)}^{2}$ are both the identity map, we find

$$
Q_{(i, k)} N_{(k, l)} P_{(l, i)}=\left(Q_{(k, l)}^{1} P_{(l, j)}^{1}\right) \otimes\left(Q_{(i, k)}^{2} P_{(k, l)}^{2}\right) .
$$

Proof Following the proof of Lemma 2.5, for $h_{j}=h_{j}^{1} \otimes h_{j}^{2} \in H_{j}^{1} \otimes H_{j}^{2}$ one finds

$$
P_{(l, j)}\left(h_{j}\right)=\left(0, P_{(l, j)}\left(h_{j}^{1}\right) \otimes h_{j}^{2}, 0\right) \in A_{l} .
$$

The image of this element of $A_{l}$ under $g_{(k, l)}^{1} \otimes g_{(k, l)}^{2}$ is precisely

$$
g_{(k, l)}^{1} P_{(l, j)}^{1}\left(h_{j}^{1}\right) \otimes g_{(k, l)}^{2}\left(h_{j}^{2}\right) \subset C_{j}^{1} \otimes\left(H_{j}^{2} \oplus A_{j}^{2}\right) .
$$

On the other hand, the domain of $C_{(i, k)}$ (where it is non-zero) is the subset

$$
0 \oplus 0 \oplus\left(H_{k}^{1} \otimes A_{k}^{2}\right) \oplus 0 \subset A_{k} .
$$

In other words, only the component of $g_{(k, l)}^{1} P_{(l, j)}^{1}\left(h_{j}^{1}\right) \otimes g_{(k, l)}^{2}\left(h_{j}^{2}\right)$ which lands in $H_{k}^{2} \otimes A_{k}^{2}$ survives under the map $Q_{(i, k)}$. The aforementioned component is precisely $Q_{(k, l)}^{1} P_{(l, j)}^{1}\left(h_{j}^{1}\right) \otimes P_{(k, l)}^{2}\left(h_{j}^{2}\right)$ and the image of this element under $Q_{(i, k)}$ is precisely $Q_{(k, l)}^{1} P_{(l, j)}^{1}\left(h_{j}^{1}\right) \otimes Q_{(i, k)}^{2} P_{(k, l)}^{2}\left(h_{j}^{2}\right)$. This completes the proof of the lemma. 


\section{A pair of exact triangles}

\subsection{The chain maps}

Let $K \subset Y$ denote a null-homologous knot and fix a Heegaard diagram

$$
\widehat{H}=\left(\Sigma, \boldsymbol{\alpha}=\left\{\alpha_{1}, \ldots, \alpha_{g}\right\}, \widehat{\boldsymbol{\beta}}=\left\{\beta_{1}, \ldots, \beta_{g-1}\right\}\right)
$$

for the knot complement $Y \backslash K$. Set $\boldsymbol{\beta}_{\bullet}=\left\{\beta_{1}^{\bullet}, \ldots, \beta_{g-1}^{\bullet}, \lambda_{\bullet}\right\}$, where $\beta_{i}^{\bullet}$ is an isotopic copy of the curve $\beta_{i}$ and $\lambda_{\bullet}$ is chosen so that the Heegaard triple $\left(\Sigma, \boldsymbol{\alpha}, \boldsymbol{\beta}_{\bullet}\right)$ corresponds to the three-manifold obtained from $Y$ by $\bullet$-surgery on the knot $K$. Choose the curves $\lambda_{0}, \lambda_{1}$ and $\lambda_{\infty}$ so that each pair of them has a unique transverse intersection point. The orientation on $K$ induces an orientation on the three curves $\lambda_{0}, \lambda_{1}$ and $\lambda_{\infty}$.

We assume that the intersection pattern of $\lambda_{0}, \lambda_{1}$ and $\lambda_{\infty}$ is one of the two patterns illustrated in Figure 1. This gives the Heegaard quadruples

$$
H=\left(\Sigma, \boldsymbol{\alpha}, \boldsymbol{\beta}_{0}, \boldsymbol{\beta}_{1}, \boldsymbol{\beta}_{\infty} ; u, v, w\right) \quad \text { and } \quad \bar{H}=\left(\Sigma, \boldsymbol{\alpha}, \boldsymbol{\beta}_{0}, \boldsymbol{\beta}_{1}, \boldsymbol{\beta}_{\infty} ; \bar{u}, \bar{v}, \bar{w}\right) .
$$

Note that there is an identification $\widehat{\mathrm{CF}}\left(\Sigma, \boldsymbol{\alpha}, \boldsymbol{\beta}_{\bullet} ; u, v, w\right)=\widehat{\mathrm{CF}}\left(\Sigma, \boldsymbol{\alpha}, \boldsymbol{\beta}_{\bullet} ; \bar{u}, \bar{v}, \bar{w}\right)$ for $\bullet \in\{0,1, \infty\}$. Moreover, for $\bullet, \star \in\{0,1, \infty\}$ the complexes $\widehat{\mathrm{CF}}\left(\Sigma, \boldsymbol{\beta}_{\bullet}, \boldsymbol{\beta}_{\star} ; u, v, w\right)$ and $\widehat{\mathrm{CF}}\left(\Sigma, \boldsymbol{\beta}_{\bullet}, \boldsymbol{\beta}_{\star} ; \bar{u}, \bar{v}, \bar{w}\right)$ are identical and the corresponding homology group is $\widehat{\mathrm{HF}}\left(\#^{g-1}\left(S^{1} \times S^{2}\right)\right)$. The top generator $\Theta=\Theta_{\bullet, \star}$ in this Heegaard Floer homology group may be used to define two holomorphic triangle maps (see Ozsváth and Szabó [12] for more details on the definition of holomorphic triangle maps).

Definition 3.1 Associated with the Heegaard triples

$$
H_{\bullet}=H \backslash \boldsymbol{\beta}_{\bullet} \quad \text { and } \quad \bar{H}_{\bullet}=\bar{H} \backslash \boldsymbol{\beta}_{\bullet},
$$

define the maps

$$
\begin{gathered}
\phi\left(H_{0}\right), \phi\left(\bar{H}_{0}\right): \widehat{\mathrm{CF}}\left(\Sigma, \boldsymbol{\alpha}, \boldsymbol{\beta}_{1} ; u, v, w\right) \longrightarrow \widehat{\mathrm{CF}}\left(\Sigma, \boldsymbol{\alpha}, \boldsymbol{\beta}_{\infty} ; u, v, w\right), \\
\phi\left(H_{1}\right), \phi\left(\bar{H}_{1}\right): \widehat{\mathrm{CF}}\left(\Sigma, \boldsymbol{\alpha}, \boldsymbol{\beta}_{\infty} ; u, v, w\right) \longrightarrow \widehat{\mathrm{CF}}\left(\Sigma, \boldsymbol{\alpha}, \boldsymbol{\beta}_{0} ; u, v, w\right), \\
\phi\left(H_{\infty}\right), \phi\left(\bar{H}_{\infty}\right): \widehat{\mathrm{CF}}\left(\Sigma, \boldsymbol{\alpha}, \boldsymbol{\beta}_{0} ; u, v, w\right) \longrightarrow \widehat{\mathrm{CF}}\left(\Sigma, \boldsymbol{\alpha}, \boldsymbol{\beta}_{1} ; u, v, w\right)
\end{gathered}
$$

to be the holomorphic triangle maps corresponding to the triply punctured Heegaard triples $H_{0}, \bar{H}_{0}, H_{1}, \bar{H}_{1}, H_{\infty}$ and $\bar{H}_{\infty}$, respectively, defined using the top generators $\Theta_{\bullet, \star}$. Denote the induced maps in homology by $\phi_{*}\left(H_{\bullet}\right)$ and $\phi_{*}\left(\bar{H}_{\bullet}\right)$ and set

$$
\mathfrak{f}_{\bullet}(K):=\phi_{*}\left(H_{\bullet}\right) \quad \text { and } \quad \overline{\mathfrak{f}}_{\bullet}(K):=\phi_{*}\left(\bar{H}_{\bullet}\right) \quad \text { for } \bullet \in\{0,1, \infty\} \text {. }
$$




\subsection{Behaviour under Heegaard moves}

The group $\widehat{\mathrm{HF}}\left(\Sigma, \boldsymbol{\alpha}, \boldsymbol{\beta}_{\bullet} ; u, v, w\right)$, denoted by $\mathbb{H}_{\bullet}(K)$, is independent of the particular Heegaard diagram used for the definition. We have thus defined the maps

$$
\mathfrak{f}_{0}(K), \overline{\mathfrak{f}}_{0}(K): \mathbb{H}_{1}(K) \rightarrow \mathbb{H}_{\infty}(K) \quad \text { and } \quad \mathfrak{f}_{\infty}(K), \overline{\mathfrak{f}}_{\infty}(K): \mathbb{H}_{0}(K) \rightarrow \mathbb{H}_{1}(K) .
$$

The definition of the map $\mathfrak{f}_{0}(K)$ depends on a Heegaard triple $\left(\Sigma, \boldsymbol{\alpha}, \boldsymbol{\beta}_{1}, \boldsymbol{\beta}_{\infty} ; u, v, w\right)$ associated with the knot $K$. Changing $H$ to another Heegaard triple changes $\mathbb{H}_{1}(K)$ and $\mathbb{H}_{\infty}(K)$ by an isomorphism which is determined by the corresponding Heegaard moves that change one Heegaard diagram to the other. We would now like to show that the corresponding change in the triangle maps $\mathfrak{f}_{0}(H)$ and $\overline{\mathfrak{f}}_{0}(H)$ respects the above isomorphisms. This justifies using the names $\mathfrak{f}_{0}(K)$ and $\overline{\mathfrak{f}}_{0}(K)$ for the above two homomorphisms. The same statement would be true for $\mathfrak{f}_{\bullet}(K)$ and $\overline{\mathfrak{f}}_{\bullet}(K)$.

Let $\{*\}=\{0,1, \infty\} \backslash\{\bullet, \star\}$. Suppose that two marked Heegaard triples

$$
H_{*}=\left(\Sigma, \boldsymbol{\alpha}, \boldsymbol{\beta}_{\bullet}, \boldsymbol{\beta}_{\star}, u, v, w\right) \quad \text { and } \quad H_{*}^{\prime}=\left(\Sigma^{\prime}, \boldsymbol{\alpha}^{\prime}, \boldsymbol{\beta}_{\bullet}^{\prime}, \boldsymbol{\beta}_{\star}^{\prime}, u^{\prime}, v^{\prime}, w^{\prime}\right)
$$

correspond to the same knot $K \subset Y$ for a pair $(\bullet, \star) \in\{(\infty, 1),(1,0)\}$. Similarly, one may consider the Heegaard diagrams $\bar{H}_{*}$ and $\bar{H}_{*}^{\prime}$. Suppose furthermore that the maps

$$
\begin{aligned}
& l_{\bullet}: \widehat{\mathrm{HF}}\left(\Sigma, \boldsymbol{\alpha}, \boldsymbol{\beta}_{\bullet} ; u, v, w\right) \longrightarrow \widehat{\mathrm{HF}}\left(\Sigma^{\prime}, \boldsymbol{\alpha}^{\prime}, \boldsymbol{\beta}_{\bullet}^{\prime} ; u^{\prime}, v^{\prime}, w^{\prime}\right), \\
& l_{\star}: \widehat{\mathrm{HF}}\left(\Sigma, \boldsymbol{\alpha}, \boldsymbol{\beta}_{\star} ; u, v, w\right) \longrightarrow \widehat{\mathrm{HF}}\left(\Sigma^{\prime}, \boldsymbol{\alpha}^{\prime}, \boldsymbol{\beta}_{\star}^{\prime} ; u^{\prime}, v^{\prime}, w^{\prime}\right)
\end{aligned}
$$

are the isomorphisms of the corresponding Heegaard Floer homology groups associated with the Heegaard moves (and the change of almost complex structure) changing one Heegaard diagram to the other.

Theorem 3.2 With the above notation fixed,

$$
\mathfrak{f}_{*}\left(H_{*}\right) \circ l_{\bullet}=l_{\star} \circ \mathfrak{f}_{*}\left(H_{*}^{\prime}\right) \quad \text { and } \quad \overline{\mathfrak{f}}_{*}\left(\bar{H}_{*}\right) \circ l_{\bullet}=l_{\star} \circ \overline{\mathfrak{f}}_{*}\left(\bar{H}_{*}^{\prime}\right) .
$$

Proof The proof consists of some standard steps in Heegaard Floer theory, which are sketched below for the Heegaard moves.

Note that the first Heegaard triple may be changed to the second Heegaard triple by a sequence of Heegaard moves, supported in the complement of the marked points, of the following types:

- Changing the almost complex structure on the surface $\Sigma$.

- Isotopies of the curves in $\boldsymbol{\alpha}$ which are supported away from a neighbourhood $U$ of $\lambda_{\bullet} \cap \lambda_{\star}$ containing the marked points $u, v$ and $w$, so that the curves in each collection remain disjoint. 
- Handle slides among the curves in $\boldsymbol{\alpha}$ supported away from $U$.

- Simultaneous handle slides among $\boldsymbol{\beta}_{\bullet} \backslash\left\{\lambda_{\bullet}\right\}$ and $\boldsymbol{\beta}_{\star} \backslash\left\{\lambda_{\star}\right\}$ supported away from $U$.

- Stabilization and destabilization of the Heegaard triple away from $U$.

The independence of the induced map in homology from the choice of the path of almost complex structures follows the corresponding argument of Ozsváth and Stipsicz [10]. Corresponding to each one of the above Heegaard moves, we obtain a holomorphic square map in the level of chain complexes, comprising of a chain homotopy map between the compositions of the chain maps we are interested in. More precisely, performing an isotopy or a handle slide in $\boldsymbol{\alpha}$ would result in a new set of simple closed curves, which may be denoted by $\boldsymbol{\alpha}^{\prime}$, by slight abuse of notation. The punctured Heegaard 4-tuple

$$
\left(\Sigma, \boldsymbol{\alpha}, \boldsymbol{\alpha}^{\prime}, \boldsymbol{\beta}_{\bullet}, \boldsymbol{\beta}_{\star} ; u, v, w\right)
$$

determines a homomorphism

$\widehat{\Phi}: \widehat{C F}\left(\Sigma, \boldsymbol{\alpha}, \boldsymbol{\alpha}^{\prime} ; u, v, w\right) \otimes \widehat{C F}\left(\Sigma, \boldsymbol{\alpha}^{\prime}, \boldsymbol{\beta}_{\bullet} ; u, v, w\right) \otimes \widehat{C F}\left(\Sigma, \boldsymbol{\beta}_{\bullet}, \boldsymbol{\beta}_{\star} ; u, v, w\right)$

$\longrightarrow \widehat{\mathrm{CF}}\left(\Sigma, \boldsymbol{\alpha}, \boldsymbol{\beta}_{\star} ; u, v, w\right)$,

which is defined by counting holomorphic squares with Maslov index -1 . Using the top closed elements in the complexes $\widehat{\mathrm{CF}}\left(\Sigma, \boldsymbol{\alpha}, \boldsymbol{\alpha}^{\prime} ; u, v, w\right)$ and $\widehat{\mathrm{CF}}\left(\Sigma, \boldsymbol{\beta}_{\bullet}, \boldsymbol{\beta}_{\star} ; u, v, w\right)$, we obtain a corresponding map

$$
\Phi: \widehat{\mathrm{CF}}\left(\Sigma, \boldsymbol{\alpha}^{\prime}, \boldsymbol{\beta}_{\bullet} ; u, v, w\right) \longrightarrow \widehat{\mathrm{CF}}\left(\Sigma, \boldsymbol{\alpha}, \boldsymbol{\beta}_{\star} ; u, v, w\right) .
$$

Let us denote the differentials of the chain complexes

$$
\widehat{\mathrm{CF}}\left(\Sigma, \boldsymbol{\alpha}^{\prime}, \boldsymbol{\beta}_{\bullet} ; u, v, w\right) \text { and } \widehat{\mathrm{CF}}\left(\Sigma, \boldsymbol{\alpha}, \boldsymbol{\beta}_{\star} ; u, v, w\right)
$$

by $d_{\alpha^{\prime}, \beta_{\bullet}}$ and $d_{\alpha, \beta_{\star}}$, respectively. The Heegaard triples $\left(\Sigma, \boldsymbol{\alpha}, \boldsymbol{\alpha}^{\prime}, \boldsymbol{\beta}_{\bullet}\right),\left(\Sigma, \boldsymbol{\alpha}, \boldsymbol{\alpha}^{\prime}, \boldsymbol{\beta}_{\star}\right)$ determine chain equivalences

$$
\begin{aligned}
& l\left(\boldsymbol{\alpha}, \boldsymbol{\alpha}^{\prime}, \boldsymbol{\beta}_{\bullet}\right): \widehat{\mathrm{CF}}\left(\Sigma, \boldsymbol{\alpha}^{\prime}, \boldsymbol{\beta}_{\bullet} ; u, v, w\right) \longrightarrow \widehat{\mathrm{CF}}\left(\Sigma, \boldsymbol{\alpha}, \boldsymbol{\beta}_{\bullet} ; u, v, w\right), \\
& \imath\left(\boldsymbol{\alpha}, \boldsymbol{\alpha}^{\prime}, \boldsymbol{\beta}_{\star}\right): \widehat{\mathrm{CF}}\left(\Sigma, \boldsymbol{\alpha}^{\prime}, \boldsymbol{\beta}_{\star} ; u, v, w\right) \longrightarrow \widehat{\mathrm{CF}}\left(\Sigma, \boldsymbol{\alpha}, \boldsymbol{\beta}_{\star} ; u, v, w\right) .
\end{aligned}
$$

Moreover, we obtain holomorphic triangle maps associated with the Heegaard triples $\left(\Sigma, \boldsymbol{\alpha}, \boldsymbol{\beta}_{\bullet}, \boldsymbol{\beta}_{\star}\right)$ and $\left(\Sigma, \boldsymbol{\alpha}^{\prime}, \boldsymbol{\beta}_{\bullet}, \boldsymbol{\beta}_{\star}\right)$, which are denoted by

$$
\begin{gathered}
\phi\left(\boldsymbol{\alpha}, \boldsymbol{\beta}_{\bullet}, \boldsymbol{\beta}_{\star}\right): \widehat{\mathrm{CF}}\left(\Sigma, \boldsymbol{\alpha}, \boldsymbol{\beta}_{\bullet} ; u, v, w\right) \longrightarrow \widehat{\mathrm{CF}}\left(\Sigma, \boldsymbol{\alpha}, \boldsymbol{\beta}_{\star} ; u, v, w\right), \\
\phi\left(\boldsymbol{\alpha}^{\prime}, \boldsymbol{\beta}_{\bullet}, \boldsymbol{\beta}_{\star}\right): \widehat{\mathrm{CF}}\left(\Sigma, \boldsymbol{\alpha}^{\prime}, \boldsymbol{\beta}_{\bullet} ; u, v, w\right) \longrightarrow \widehat{\mathrm{CF}}\left(\Sigma, \boldsymbol{\alpha}^{\prime}, \boldsymbol{\beta}_{\star} ; u, v, w\right) .
\end{gathered}
$$


Considering different types of degenerations for a square of Maslov index 0, we obtain the relation

$$
d_{\alpha \beta_{\star}} \circ \Phi+\Phi \circ d_{\alpha^{\prime} \beta_{\bullet}}=l\left(\boldsymbol{\alpha}, \boldsymbol{\alpha}^{\prime}, \boldsymbol{\beta}_{\star}\right) \circ \phi\left(\boldsymbol{\alpha}, \boldsymbol{\beta}_{\bullet}, \boldsymbol{\beta}_{\star}\right)+\phi\left(\boldsymbol{\alpha}^{\prime}, \boldsymbol{\beta}_{\bullet}, \boldsymbol{\beta}_{\star}\right) \circ l\left(\boldsymbol{\alpha}, \boldsymbol{\alpha}^{\prime}, \boldsymbol{\beta}_{\bullet}\right) .
$$

The induced relation in homology gives the claim for the invariance of $\phi_{*}\left(H_{*}\right)$ under handle slides in $\alpha$. The corresponding argument for $\phi_{*}\left(\bar{H}_{*}\right)$ is done by changing the marked points.

The invariance under handle slides among the $\beta$-curves is proved similarly, and we only highlight the important modifications. Let $\boldsymbol{\beta}_{\bullet}^{\prime}$ and $\boldsymbol{\beta}_{\star}^{\prime}$ be obtained from $\boldsymbol{\beta}_{\bullet}$ and $\boldsymbol{\beta}_{\star}$ by handle slides which correspond to a handle slide in $\widehat{\boldsymbol{\beta}}$. We thus have the following square of chain maps:

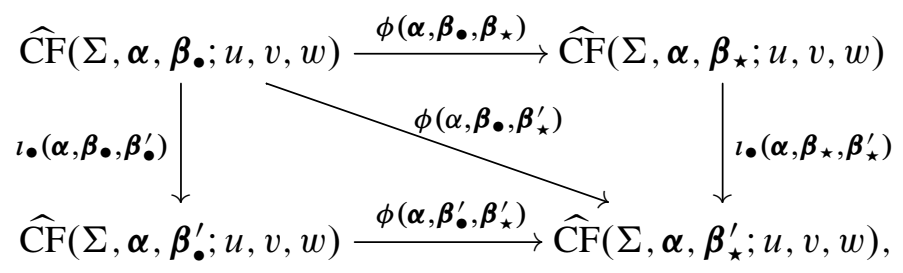

while the quadruples $\left(\Sigma, \boldsymbol{\alpha}, \boldsymbol{\beta}_{\bullet}, \boldsymbol{\beta}_{\bullet}^{\prime}, \boldsymbol{\beta}_{\star}^{\prime} ; u, v, w\right)$ and $\left(\Sigma, \boldsymbol{\alpha}, \boldsymbol{\beta}_{\bullet}, \boldsymbol{\beta}_{\star}, \boldsymbol{\beta}_{\star}^{\prime} ; u, v, w\right)$ determine a pair of holomorphic square maps

$$
\Phi_{1}, \Phi_{2}: \widehat{\mathrm{CF}}\left(\alpha, \boldsymbol{\beta}_{\bullet} ; u, v, w\right) \longrightarrow \widehat{\mathrm{CF}}\left(\boldsymbol{\alpha}, \boldsymbol{\beta}_{\star}^{\prime} ; u, v, w\right) .
$$

Considering different possible degenerations of holomorphic squares of Maslov index 0 gives the relations

$$
\begin{aligned}
& d_{\alpha \beta_{\star}^{\prime}} \circ \Phi_{1}+\Phi_{1} \circ d_{\alpha \beta_{\bullet}}=\phi\left(\boldsymbol{\alpha}, \boldsymbol{\beta}_{\bullet}^{\prime}, \boldsymbol{\beta}_{\star}^{\prime}\right) \circ l\left(\boldsymbol{\alpha}, \boldsymbol{\beta}_{\bullet}, \boldsymbol{\beta}_{\bullet}^{\prime}\right)+\phi\left(\boldsymbol{\alpha}, \boldsymbol{\beta}_{\bullet}, \boldsymbol{\beta}_{\star}^{\prime}\right), \\
& d_{\alpha \beta_{\star}^{\prime}} \circ \Phi_{2}+\Phi_{2} \circ d_{\alpha \beta_{\bullet}}=l\left(\boldsymbol{\alpha}, \boldsymbol{\beta}_{\star}, \boldsymbol{\beta}_{\star}^{\prime}\right) \circ \phi\left(\boldsymbol{\alpha}, \boldsymbol{\beta}_{\bullet}, \boldsymbol{\beta}_{\star}\right)+\phi\left(\boldsymbol{\alpha}, \boldsymbol{\beta}_{\bullet}, \boldsymbol{\beta}_{\star}^{\prime}\right) .
\end{aligned}
$$

If we set $\Phi=\Phi_{1}+\Phi_{2}$ we thus find

$$
d_{\alpha \beta_{\star}^{\prime}} \circ \Phi+\Phi \circ d_{\alpha \beta_{\bullet}}=\phi\left(\boldsymbol{\alpha}, \boldsymbol{\beta}_{\bullet}^{\prime}, \boldsymbol{\beta}_{\star}^{\prime}\right) \circ l\left(\boldsymbol{\alpha}, \boldsymbol{\beta}_{\bullet}, \boldsymbol{\beta}_{\bullet}^{\prime}\right)+l\left(\boldsymbol{\alpha}, \boldsymbol{\beta}_{\star}, \boldsymbol{\beta}_{\star}^{\prime}\right) \circ \phi\left(\boldsymbol{\alpha}, \boldsymbol{\beta}_{\bullet}, \boldsymbol{\beta}_{\star}\right),
$$

which completes the proof of the invariance under handle slides of the $\beta$-curves for $\phi_{*}\left(H_{*}\right)$. The argument for $\phi\left(\bar{H}_{*}\right)$ is completely similar.

The proof of the invariance under stabilization and destabilization follows the general argument of [10] as well.

Remark 3.3 This theorem should be compared with the naturality theorem of Ozsvath and Stipsicz [10]. 
Lemma 3.4 With the above notation fixed, the triangles
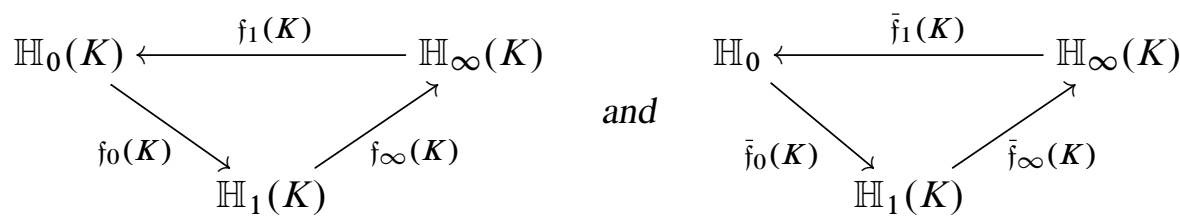

are both exact.

Proof The more general forms of exact triangles associated with pointed Heegaard diagrams are discussed by Alishahi and Eftekhary [1, Section 9], using a generalization of Lemma 4.4 of Ozsváth and Szabó [13]. The arguments are rather standard and are omitted from the paper. The only remark is that if the intersection pattern of $\lambda_{0}, \lambda_{1}$ and $\lambda_{\infty}$ follows the left-hand side of Figure 1, the contributing holomorphic triangles for $\left(\Sigma, \boldsymbol{\beta}_{0}, \boldsymbol{\beta}_{1}, \boldsymbol{\beta}_{\infty} ; u, v, w\right)$ come in cancelling pairs, allowing us to follow the standard arguments. For the Heegaard triple $\left(\Sigma, \boldsymbol{\beta}_{0}, \boldsymbol{\beta}_{1}, \boldsymbol{\beta}_{\infty} ; \bar{u}, \bar{v}, \bar{w}\right)$, however, there is a unique contributing triangle class, which corresponds to the small triangle bounded between the three curves, which implies that the corresponding triangle map takes $\Theta_{0,1} \otimes \Theta_{1, \infty}$ to $\Theta_{0, \infty}$. Nevertheless, the position of the punctures in this case implies that the map $\phi\left(\boldsymbol{\alpha}, \boldsymbol{\beta}_{0}, \boldsymbol{\beta}_{\infty} ; \bar{u}, \bar{v}, \bar{w}\right)$ that is defined using $\Theta_{0,1}$ is trivial (unlike $\left.\phi\left(\boldsymbol{\alpha}, \boldsymbol{\beta}_{\infty}, \boldsymbol{\beta}_{0} ; \bar{u}, \bar{v}, \bar{w}\right)\right)$. From here, the rest of the argument is standard.

By exactness of the triangles in $(3), \operatorname{Ker}\left(\mathfrak{f}_{\infty}(K)\right)$ is isomorphic to $\operatorname{Coker}\left(\mathfrak{f}_{0}(K)\right)$ while $\operatorname{Ker}\left(\overline{\mathfrak{f}}_{\infty}(K)\right)$ is isomorphic to $\operatorname{Coker}\left(\overline{\mathfrak{f}}_{0}(K)\right)$. Furthermore, the first isomorphism is induced by the natural chain map $\mathfrak{f}_{1}(K)$ while the second isomorphism is induced by $\overline{\mathfrak{f}}_{1}(K)$. Let $\theta(K): \mathbb{H}_{0}(K) \rightarrow \mathbb{H}_{\infty}(K)$ denote a map which has the same rank as $\mathfrak{f}_{1}(K)$ and induces the inverse of the isomorphism

$$
\mathfrak{f}_{1}(K): \operatorname{Ker}\left(\mathfrak{f}_{\infty}(K)\right) \longrightarrow \operatorname{Coker}\left(\mathfrak{f}_{1}(K)\right),
$$

while $\bar{\theta}(K): \mathbb{H}_{0}(K) \rightarrow \mathbb{H}_{\infty}(K)$ denotes a map which has the same rank as $\overline{\mathfrak{f}}_{1}(K)$ and induces the inverse of the isomorphism

$$
\overline{\mathfrak{f}}_{1}(K): \operatorname{Ker}\left(\mathfrak{f}_{\infty}(K)\right) \longrightarrow \operatorname{Coker}\left(\mathfrak{f}_{1}(K)\right) .
$$

The choice of the maps $\theta(K)$ and $\bar{\theta}(K)$ are of course not unique. If

$$
\phi_{\infty}=\phi\left(\boldsymbol{\alpha}, \boldsymbol{\beta}_{0}, \boldsymbol{\beta}_{1} ; u, v, w\right) \quad \text { and } \quad \phi_{0}=\phi\left(\boldsymbol{\alpha}, \boldsymbol{\beta}_{1}, \boldsymbol{\beta}_{\infty} ; u, v, w\right)
$$

denote the triangle maps associated with the punctured Heegaard triples

$$
\left(\Sigma, \boldsymbol{\alpha}, \boldsymbol{\beta}_{0}, \boldsymbol{\beta}_{1} ; u, v, w\right) \text { and }\left(\Sigma, \boldsymbol{\alpha}, \boldsymbol{\beta}_{1}, \boldsymbol{\beta}_{\infty} ; u, v, w\right),
$$


as above, the map $\theta(K)$ is in fact the correction term, in the sense of Lemma 2.4, associated with the sequence (or, in fact, graph of complexes)

$$
\widehat{\mathrm{CF}}\left(\Sigma, \boldsymbol{\alpha}, \boldsymbol{\beta}_{0} ; u, v, w\right) \stackrel{\phi_{\infty}}{\longrightarrow} \widehat{\mathrm{CF}}\left(\Sigma, \boldsymbol{\alpha}, \boldsymbol{\beta}_{1} ; u, v, w\right) \stackrel{\phi_{0}}{\longrightarrow} \widehat{\mathrm{CF}}\left(\Sigma, \boldsymbol{\alpha}, \boldsymbol{\beta}_{\infty} ; u, v, w\right) .
$$

Similarly, $\bar{\theta}(K)$ corresponds to the sequence

$$
\widehat{\mathrm{CF}}\left(\Sigma, \boldsymbol{\alpha}, \boldsymbol{\beta}_{0} ; \bar{u}, \bar{v}, \bar{w}\right) \stackrel{\bar{\phi}_{\infty}}{\longrightarrow} \widehat{\mathrm{CF}}\left(\Sigma, \boldsymbol{\alpha}, \boldsymbol{\beta}_{1} ; \bar{u}, \bar{v}, \bar{w}\right) \stackrel{\bar{\phi}_{0}}{\longrightarrow} \widehat{\mathrm{CF}}\left(\Sigma, \boldsymbol{\alpha}, \boldsymbol{\beta}_{\infty} ; \bar{u}, \bar{v}, \bar{w}\right),
$$

where

$$
\bar{\phi}_{\infty}=\phi\left(\boldsymbol{\alpha}, \boldsymbol{\beta}_{0}, \boldsymbol{\beta}_{1} ; \bar{u}, \bar{v}, \bar{w}\right) \quad \text { and } \quad \bar{\phi}_{0}=\phi\left(\boldsymbol{\alpha}, \boldsymbol{\beta}_{1}, \boldsymbol{\beta}_{\infty} ; \bar{u}, \bar{v}, \bar{w}\right)
$$

\subsection{Some properties of the maps $\mathfrak{f}_{\bullet}(K)$ and $\overline{\mathfrak{f}}_{\bullet}(K)$}

Our first observation is that changing the orientation of the knot $K$ and, correspondingly that of $K_{1}$ and $K_{0}$, corresponds to changing the markings $u, v, w$ with $\bar{u}, \bar{v}, \bar{w}$ in Figure 1. Suppose that $\left(\Sigma, \boldsymbol{\alpha}, \boldsymbol{\beta} ; z_{1}, z_{2}\right)$ represents $K_{\bullet}$, meaning that an oriented longitude for $K_{\bullet}$ is constructed from gluing an oriented arc on $\Sigma$ from $z_{1}$ to $z_{2}$ in the complement of $\alpha$ and an oriented arc on $\Sigma$ from $z_{2}$ to $z_{1}$ in the complement of $\boldsymbol{\beta}$. Then $\left(\Sigma, \boldsymbol{\alpha}, \boldsymbol{\beta} ; z_{2}, z_{1}\right)$ is a Heegaard diagram for $-K_{\bullet}$ (the knot $K_{\bullet}$ with the reverse orientation) while $\left(-\Sigma, \boldsymbol{\beta}, \boldsymbol{\alpha} ; z_{2}, z_{1}\right)$ is a Heegaard diagram for $K_{\bullet}$. The chain complexes associated with the above three Heegaard diagrams are identical. Heegaard moves give chain homotopy equivalences

$$
\tau_{\bullet}(K): \widehat{\mathrm{CF}}\left(\Sigma, \boldsymbol{\alpha}, \boldsymbol{\beta} ; z_{1}, z_{2}\right) \longrightarrow \widehat{\mathrm{CF}}\left(-\Sigma, \boldsymbol{\beta}, \boldsymbol{\alpha} ; z_{2}, z_{1}\right)=\widehat{\mathrm{CF}}\left(\Sigma, \boldsymbol{\alpha}, \boldsymbol{\beta} ; z_{1}, z_{2}\right) .
$$

These chain homotopy equivalences induce the involutions

$$
\tau_{\bullet}(K): \mathbb{H}_{\bullet}(K) \longrightarrow \mathbb{H}_{\bullet}(K), \quad \bullet \in\{0,1, \infty\} .
$$

In terms of these isomorphisms,

$$
\begin{aligned}
\overline{\mathfrak{f}}_{0}(K) & =\tau_{\infty}(K) \circ \mathfrak{f}_{0}(K) \circ \tau_{1}(K), \\
\overline{\mathfrak{f}}_{1}(K) & =\tau_{0}(K) \circ \mathfrak{f}_{1}(K) \circ \tau_{\infty}(K), \\
\overline{\mathfrak{f}}_{\infty}(K) & =\tau_{1}(K) \circ \mathfrak{f}_{\infty}(K) \circ \tau_{0}(K) .
\end{aligned}
$$

Note however, that the equality $\bar{\theta}(K)=\tau_{\infty}(K) \theta(K) \tau_{0}(K)$ is only satisfied for the induced maps from $\operatorname{Ker}\left(\overline{\mathfrak{f}}_{\infty}(K)\right)$ to $\operatorname{Coker}\left(\overline{\mathfrak{f}}_{0}(K)\right)$. 
The exactness of the sequences in (3) implies that, in appropriate decompositions

$$
\begin{aligned}
\mathbb{H}_{0}(K) & =\frac{\mathbb{H}_{0}(K)}{\operatorname{Ker}\left(\mathfrak{f}_{\infty}(K)\right)} \oplus \operatorname{Ker}\left(\mathfrak{f}_{\infty}(K)\right)=: \mathbb{A}_{\infty}(K) \oplus \mathbb{A}_{1}(K), \\
\mathbb{H}_{1}(K) & =\frac{\mathbb{H}_{1}(K)}{\operatorname{Ker}\left(\mathfrak{f}_{0}(K)\right)} \oplus \operatorname{Ker}\left(\mathfrak{f}_{0}(K)\right)=: \mathbb{A}_{0}(K) \oplus \mathbb{A}_{\infty}(K), \\
\mathbb{H}_{\infty}(K) & =\frac{\mathbb{H}_{\infty}(K)}{\operatorname{Ker}\left(\mathfrak{f}_{1}(K)\right)} \oplus \operatorname{Ker}\left(\mathfrak{f}_{1}(K)\right)=: \mathbb{A}_{1}(K) \oplus \mathbb{A}_{0}(K),
\end{aligned}
$$

we have

$$
\mathfrak{f}_{\bullet}(K)=\left(\begin{array}{cc}
0 & 0 \\
I_{a_{\bullet}(K)} & 0
\end{array}\right),
$$

where $a_{\bullet}(K)$ denotes the rank of $\mathbb{A}_{\bullet}(K)$ for every $\bullet \in\{0,1, \infty\}$. In this basis we may present the matrices $\tau_{\bullet}(K)$ as

$$
\tau_{\bullet}(K)=\left(\begin{array}{ll}
A_{\bullet}(K) & B_{\bullet}(K) \\
C_{\bullet}(K) & D_{\bullet}(K)
\end{array}\right), \quad \bullet \in\{0,1, \infty\} .
$$

The map $B_{0}(K)$ corresponds to the induced map

$$
\tau_{0}(K): \operatorname{Ker}\left(\mathfrak{f}_{\infty}(K)\right) \longrightarrow \frac{\mathbb{H}_{0}(K)}{\operatorname{Ker}\left(\mathfrak{f}_{\infty}(K)\right)} .
$$

The decomposition $\mathbb{H}_{0}(K)=\mathbb{A}_{\infty}(K) \oplus \mathbb{A}_{1}(K)$ may be modified using a change of basis of the form $P_{X}=\left(\begin{array}{rr}I & 0 \\ -X & I\end{array}\right)$, which does not change the block presentations of the maps $\mathfrak{f}_{\infty}(K)$ and $\mathfrak{f}_{1}(K)$. In the new basis, $\tau_{0}(K)$ has the following presentation:

$$
\begin{aligned}
\tau_{0}(K) & =\left(\begin{array}{rr}
I & 0 \\
-X & I
\end{array}\right)\left(\begin{array}{cc}
A_{0}(K) & B_{0}(K) \\
C_{0}(K) & D_{0}(K)
\end{array}\right)\left(\begin{array}{cc}
I & 0 \\
-X & I
\end{array}\right) \\
& =\left(\begin{array}{cc}
A_{0}(K)-B_{0}(K) X & B_{0}(K) \\
\star & -X B_{0}(K)+D_{0}(K)
\end{array}\right)
\end{aligned}
$$

If $B_{0}(K)$ is injective we may thus assume that $D_{0}(K)=0$, while if $B_{0}(K)$ is surjective we may assume that $A_{0}(K)=0$. With similar reasoning, if $B_{\bullet}(K)$ is injective we may assume that $D_{\bullet}(K)=0$, while if $B_{\bullet}(K)$ is surjective we may assume that $A_{\bullet}(K)=0$. In the above decompositions for $\mathbb{H}_{\bullet}(K)$, the map $\theta(K): \mathbb{H}_{0}(K) \rightarrow \mathbb{H}_{\infty}(K)$ takes the form

$$
\theta(K)=\left(\begin{array}{ll}
X & I \\
Z & Y
\end{array}\right)
$$

since the induced map from $\mathbb{A}_{1}(K) \subset \mathbb{H}_{0}(K)$ to $\mathbb{A}_{1}(K) \subset \mathbb{H}_{\infty}(K)$ is the inverse of the map induced by $\mathfrak{f}_{1}(K)$, ie the identity. Moreover, since the rank of $\theta(K)$ is the same as the rank of $\mathfrak{f}_{1}(K)$, we conclude that $Z=Y X$. Applying the change of basis 
$P_{Y}$ on $\mathbb{H}_{0}(K)$ and the corresponding change of basis $P_{X}$ on $\mathbb{H}_{\infty}(K), \theta(K)$ takes the form

$$
\left(\begin{array}{rr}
I & 0 \\
-Y & I
\end{array}\right)\left(\begin{array}{cc}
X & I \\
Y X & Y
\end{array}\right)\left(\begin{array}{rr}
I & 0 \\
-X & I
\end{array}\right)=\left(\begin{array}{ll}
0 & I \\
0 & 0
\end{array}\right)
$$

It is thus possible to choose the above decompositions so that $\theta(K)=\left(\begin{array}{ll}0 & I \\ 0 & 0\end{array}\right)$. If this is the case, the $2 \times 2$ presentation of $\tau_{\infty}(K) \bar{\theta}(K) \tau_{0}(K)$ will be of the form

$$
\tau_{\infty}(K) \bar{\theta}(K) \tau_{0}(K)=\left(\begin{array}{cc}
M & I \\
Q & P
\end{array}\right)
$$

and, since the ranks of $\theta(K)$ and $\bar{\theta}(K)$ are the same, we find $Q=P M$.

\subsection{Relative Spin ${ }^{c}$ structures}

The vector spaces $\mathbb{H}_{\infty}(K)$ and $\mathbb{H}_{1}(K)$ are naturally decomposed by relative $\operatorname{Spin}^{c}$ classes in

$$
\underline{\operatorname{Spin}^{c}}(Y, K)=\underline{\operatorname{Spin}^{c}}\left(Y_{1}(K), K_{1}\right)=\mathbb{Z},
$$

where the identification with $\mathbb{Z}$ is made using the first Chern class (divided by 2 ). Similarly, the relative $\operatorname{Spin}^{c}$ classes corresponding to $K_{0}$ are identified with $\frac{1}{2}+\mathbb{Z}$. Thus,

$$
\mathbb{H}_{\bullet}(K)=\bigoplus_{i \in \mathbb{Z}} \mathbb{H}_{\bullet}(K, i), \quad \bullet \in\{1, \infty\}, \quad \text { and } \quad \mathbb{H}_{0}(K)=\bigoplus_{j \in \frac{1}{2}+\mathbb{Z}} \mathbb{H}_{0}(K, j)
$$

Note that $\tau_{\bullet}(K)$ takes $\mathbb{H}_{\bullet}(K, i)$ isomorphically to $\mathbb{H}_{\bullet}(K,-i)$ for $\bullet=0,1, \infty$.

Let $H_{0}=\left(\Sigma, \boldsymbol{\alpha}, \boldsymbol{\beta}_{1}, \boldsymbol{\beta}_{\infty} ; u, v, w\right)$ be a Heegaard triple used for defining $\mathfrak{f}_{0}(K)$. If $\boldsymbol{x} \in \mathbb{T}_{\alpha} \cap \mathbb{T}_{\beta_{1}}$ and $\boldsymbol{y} \in \mathbb{T}_{\alpha} \cap \mathbb{T}_{\beta_{\infty}}$ are two generators connected by a triangle class $\Delta \in \pi_{2}\left(\boldsymbol{x}, \Theta_{1, \infty}, \boldsymbol{y}\right)$ with $n_{u}(\Delta)=n_{w}(\Delta)=0$ (as observed in the surgery exact sequences of [14]), then $c_{1}\left(\underline{\mathfrak{s}}_{u}, w(\boldsymbol{x})\right)=c_{1}\left(\mathfrak{s}_{u}, v(\boldsymbol{y})\right)$. This observation, together with (4) imply that the maps $\mathfrak{f}_{0}(K)$ and $\overline{\mathfrak{f}}_{0}(K)$ are decomposed as

$$
\begin{aligned}
& \mathfrak{f}_{0}(K)=\bigoplus_{i \in \mathbb{Z}} \mathfrak{f}_{0}(K, i), \quad \mathfrak{f}_{0}(K, i): \mathbb{H}_{1}(K, i) \longrightarrow \mathbb{H}_{\infty}(K, i), \\
& \overline{\mathfrak{f}}_{0}(K)=\bigoplus_{i \in \mathbb{Z}} \overline{\mathfrak{f}}_{0}(K, i), \quad \overline{\mathfrak{f}}_{0}(K, i): \mathbb{H}_{1}(K, i) \longrightarrow \mathbb{H}_{\infty}(K, i) .
\end{aligned}
$$

The map $\overline{\mathfrak{f}}_{\infty}(K): \mathbb{H}_{0}(K) \rightarrow \mathbb{H}_{1}(K)$ drops the $\operatorname{Spin}^{c}$ grading by $\frac{1}{2}$, while the map $\mathfrak{f}_{\infty}(K): \mathbb{H}_{1}(K) \rightarrow \mathbb{H}_{0}(K)$ increases the Spin ${ }^{c}$ grading by $\frac{1}{2}$. The corresponding 
decompositions are thus

$$
\begin{aligned}
& \mathfrak{f}_{\infty}(K)=\bigoplus_{i \in \mathbb{Z}} \mathfrak{f}_{\infty}(K, i), \quad \mathfrak{f}_{\infty}(K, i): \mathbb{H}_{0}\left(K, i-\frac{1}{2}\right) \longrightarrow \mathbb{H}_{1}(K, i), \\
& \overline{\mathfrak{f}}_{\infty}(K)=\bigoplus_{i \in \mathbb{Z}} \overline{\mathfrak{f}}_{\infty}(K, i), \quad \overline{\mathfrak{f}}_{\infty}(K, i): \mathbb{H}_{0}\left(K, i+\frac{1}{2}\right) \longrightarrow \mathbb{H}_{1}(K, i)
\end{aligned}
$$

In particular, for a knot $K$ of genus $g$ the maps $\overline{\mathfrak{f}}_{\infty}(K, g)$ and $\mathfrak{f}_{\infty}(K,-g)$ are trivial, since $\mathbb{H}_{0}\left(K, g+\frac{1}{2}\right)=\mathbb{H}_{0}\left(K,-g-\frac{1}{2}\right)=0$ by [2, Theorem 3.2]. Moreover,

$$
\begin{array}{ll}
\mathfrak{f}_{1}(K)=\bigoplus_{i \in \mathbb{Z}} \mathfrak{f}_{1}(K, i), \quad \mathfrak{f}_{1}(K, i): \mathbb{H}_{\infty}(K, i) \longrightarrow \mathbb{H}_{0}\left(K, i-\frac{1}{2}\right), \\
\overline{\mathfrak{f}}_{1}(K)=\bigoplus_{i \in \mathbb{Z}} \overline{\mathfrak{f}}_{1}(K, i), \quad \overline{\mathfrak{f}}_{1}(K, i): \mathbb{H}_{\infty}(K, i) \longrightarrow \mathbb{H}_{0}\left(K, i+\frac{1}{2}\right) .
\end{array}
$$

Let us now assume that $\left(\Sigma, \boldsymbol{\alpha}, \boldsymbol{\beta}_{0}, \boldsymbol{\beta}_{1}, \boldsymbol{\beta}_{\infty} ; u, v, w\right)$ is one of the Heegaard quadruples illustrated in Figure 1. If we drop the marked point $u$ (resp. the marked point $w$ ) from the Heegaard diagram, associated with either of the two resulting punctured Heegaard quadruples we obtain a triangle of chain maps:

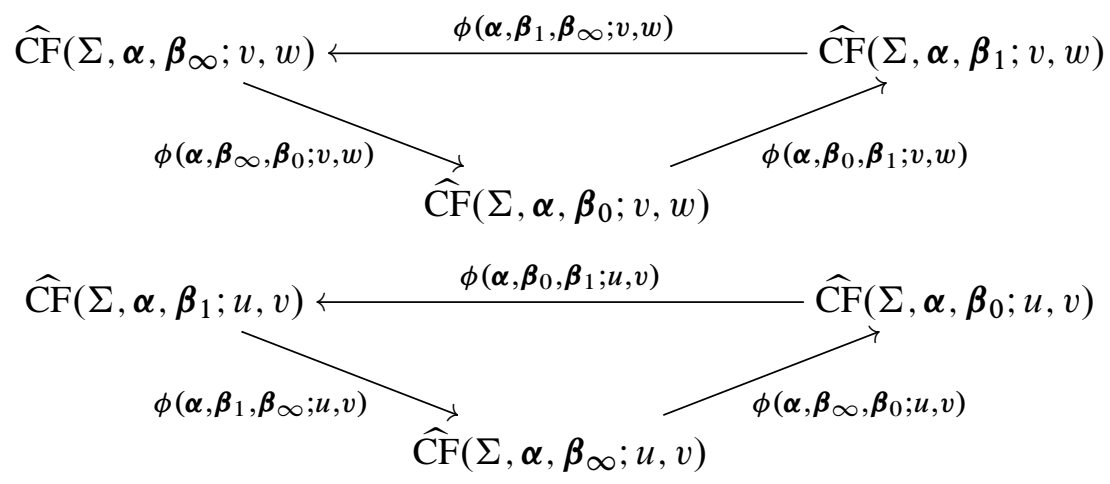

The domain of any holomorphic triangle which contributes to $\phi\left(\boldsymbol{\alpha}, \boldsymbol{\beta}_{1}, \boldsymbol{\beta}_{\infty} ; v, w\right)$ has coefficient 1 precisely at one of the base points $u$ and $\bar{u}$, and coefficient 0 at the other one. In other words,

$$
\begin{aligned}
\phi\left(\boldsymbol{\alpha}, \boldsymbol{\beta}_{1}, \boldsymbol{\beta}_{\infty} ; v, w\right) & =\phi\left(\boldsymbol{\alpha}, \boldsymbol{\beta}_{1}, \boldsymbol{\beta}_{\infty} ; u, v, w\right)+\phi\left(\boldsymbol{\alpha}, \boldsymbol{\beta}_{1}, \boldsymbol{\beta}_{\infty} ; \bar{u}, v, w\right) \\
& =\phi\left(\boldsymbol{\alpha}, \boldsymbol{\beta}_{1}, \boldsymbol{\beta}_{\infty} ; u, v, w\right)+\phi\left(\boldsymbol{\alpha}, \boldsymbol{\beta}_{1}, \boldsymbol{\beta}_{\infty} ; \bar{u}, \bar{v}, \bar{w}\right)
\end{aligned}
$$

A similar argument implies that

$$
\phi\left(\boldsymbol{\alpha}, \boldsymbol{\beta}_{0}, \boldsymbol{\beta}_{1} ; u, v\right)=\phi\left(\boldsymbol{\alpha}, \boldsymbol{\beta}_{0}, \boldsymbol{\beta}_{1} ; u, v, w\right)+\phi\left(\boldsymbol{\alpha}, \boldsymbol{\beta}_{0}, \boldsymbol{\beta}_{1} ; \bar{u}, \bar{v}, \bar{w}\right) .
$$


We thus obtain the following two exact triangles, respectively:
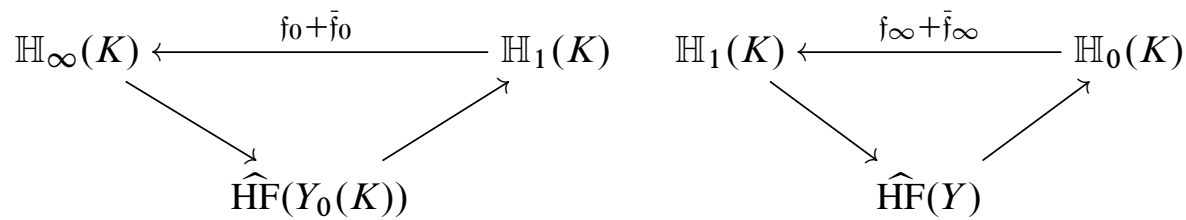

where $\mathfrak{f}_{\bullet}=\mathfrak{f}_{\bullet}(K)$ and $\overline{\mathfrak{f}}_{\bullet}=\overline{\mathfrak{f}}_{\bullet}(K)$. The exact triangles in (3) and (6) may be used to deduce the following conclusions regarding the ranks of the chain maps:

$$
\begin{array}{r}
\operatorname{rnk}\left(\mathfrak{f}_{\bullet}(K)\right)=\operatorname{rnk}\left(\overline{\mathfrak{f}}_{\bullet}(K)\right)=\frac{1}{2}\left(h_{\infty}(K)+h_{1}(K)+h_{0}(K)-2 h_{\bullet}(K)\right), \\
\operatorname{rnk}\left(\mathfrak{f}_{\bullet}(K)+\overline{\mathfrak{f}}_{\bullet}(K)\right)=\frac{1}{2}\left(h_{\infty}(K)+h_{1}(K)+h_{0}(K)-y_{\bullet}(K)-h_{\bullet}(K)\right),
\end{array}
$$

where $h_{\bullet}(K)$ denotes the rank of $\mathbb{H}_{\bullet}(K)$ and $y_{\bullet}(K)$ denotes the rank of $\widehat{\mathrm{HF}}\left(Y_{\bullet}(K)\right)$.

\section{Combinatorial presentation of the exact triangles}

\subsection{Heegaard diagrams for knot complements}

The aim of this subsection is to construct Heegaard diagrams of particular type associated with a knot $K$ inside a three-manifold $Y$, so that the chain complexes $C_{\bullet}(K)$ and the chain maps $\mathfrak{f}_{\bullet}(K)$ and $\overline{\mathfrak{f}}_{\bullet}(K)$ may all be described combinatorially.

Let us assume that a framed longitude $\hat{\lambda}$ for $K$ is given as a simple closed curve on the torus boundary of $Y \backslash \operatorname{nd}(K)$. Together with the meridian $\hat{\mu}$ of the knot $K, \hat{\lambda}$ gives a parametrization of the boundary of $Y \backslash \operatorname{nd}(K)$. It also determines the three-manifold $Y_{\hat{\lambda}}(K)$ obtained by surgery on $K$. The curves $\hat{\mu}$ and $\hat{\lambda}$ thus give $Y \backslash \operatorname{nd}(K)$ the structure of a bordered three-manifold. As such, we remind the reader that a nice Heegaard diagram

$$
\left(\Sigma, \boldsymbol{\alpha}=\left\{\alpha_{1}, \ldots, \alpha_{g}\right\}, \widehat{\boldsymbol{\beta}}=\left\{\beta_{1}, \ldots, \beta_{g-1}\right\}, \mu, \lambda ; z\right)
$$

for the bordered three-manifold determined by $(Y, K)$ and $\hat{\lambda}$ consists of a surface $\Sigma$ of genus $g$, a $g$-tuple of disjoint simple closed curves $\alpha$, a $(g-1)$-tuple of disjoint simple closed curves $\hat{\boldsymbol{\beta}}$, a pair of simple closed curves $\mu$ and $\lambda$ disjoint from $\hat{\boldsymbol{\beta}}$ which intersect in a single transverse point, and a marked point $z$ in the complement of all curves in $\Sigma$. The data satisfies the following conditions:

- The diagram $(\Sigma, \boldsymbol{\alpha}, \widehat{\boldsymbol{\beta}})$ corresponds to $Y \backslash \operatorname{nd}(K)$, while $(\Sigma, \boldsymbol{\alpha}, \hat{\boldsymbol{\beta}} \cup\{\mu\})$ and $(\Sigma, \boldsymbol{\alpha}, \widehat{\boldsymbol{\beta}} \cup\{\lambda\})$ correspond to the three-manifolds $Y$ and $Y_{\hat{\lambda}}(K)$, respectively. 
- All domains in $\Sigma \backslash(\boldsymbol{\alpha} \cup \widehat{\boldsymbol{\beta}} \cup\{\mu, \lambda\})$ are either bigons, triangles or rectangles, except for the domain $D_{z}$ containing the marked point $z$, which is a $(2 N+1)$ gon for some integer $N$. In particular, $D_{z}$ contains the single intersection point of $\mu$ and $\lambda$ as a corner.

- Every curve $\beta_{i} \in \hat{\boldsymbol{\beta}}$ contains at least one of the $2 N+1$ edges of $D_{z}$.

Nice Heegaard diagrams exist by Lipshitz, Ozsváth and Thurston [6, Proposition 8.2]. However, two remarks are necessary here. First, note that in the aforementioned proposition the roles of the $\alpha$ - and $\beta$-curves is the opposite of our convention. In particular, the curves $\mu$ and $\lambda$ are $\alpha$-curves in [6]. The second point is that the third condition above is a priori not guaranteed by [6, Proposition 8.2]. However, if $\beta_{i}$ does not contain any of the edges of $D_{z}$, all neighbouring regions of $\beta_{i}$ would be bigons or rectangles. Since $\beta_{i}$ is homotopically non-trivial, a computation of the Euler characteristic for the neighbourhood of $\beta_{i}$ (the union of all regions which are neighbours of $\beta_{i}$ ) implies that all neighbouring regions of $\beta_{i}$ are rectangles. However, this in turn implies that, for some $j \neq i, \beta_{j}$ is parallel (and thus homologous) to $\beta_{i}$, a contradiction. Thus, the third condition is also guaranteed by [6, Proposition 8.2].

The picture on the top of Figure 3 describes a surface $\widehat{\Sigma}_{1}$ of genus 4 . The opposite edges of the rectangle are identified and the pairs of yellow and red circles are also glued together (using a horizontal reflection). The pair of green circles is identified using a vertical reflection. The solid red curves are labelled $\mu$ and $\lambda$, which meet in a single transverse point $O$. The green domains glue together and form a disk $D$ on $\widehat{\Sigma}_{1}$. We set $\Sigma_{1}=\widehat{\Sigma}_{1} \backslash \operatorname{Int}(D)$. The dashed blue curves in $\Sigma_{1}$ correspond to the $\beta$-curves, while the solid black curves correspond to the $\alpha$-curves. The $\alpha$-and $\beta$-curves may have boundary in $\partial D$.

Lemma 4.1 Let $K$ be a knot inside a three-manifold $Y$ together with an arbitrary framing. Then there is a nice Heegaard diagram $(\Sigma, \boldsymbol{\alpha}, \widehat{\boldsymbol{\beta}} \cup\{\mu, \lambda\}, z)$ for the corresponding bordered three-manifold with the following properties:

- $\Sigma=\Sigma_{1} \amalg_{\partial \Sigma_{1}=\partial \Sigma_{2}} \Sigma_{2}$, where $\widehat{\Sigma}_{1}$ is the surface of genus 4 illustrated in Figure 3 and $\Sigma_{2}$ is a surface with one boundary component.

- The arcs in $\alpha \cap \Sigma_{1}$ are identified with the solid black curves in Figure 3, while the arcs in $\widehat{\boldsymbol{\beta}} \cap \Sigma_{1}$ are identified with the dashed blue curves in Figure 3.

- The curves $\mu$ and $\lambda$ correspond to the bold red curves on $\Sigma_{1}$.

- The domains on $\Sigma_{1}$ which contain the bold markings belong to the connected component $D_{z}$ in $\Sigma \backslash(\boldsymbol{\alpha} \cup \widehat{\boldsymbol{\beta}} \cup \lambda \cup \mu)$ which contains $z$. 

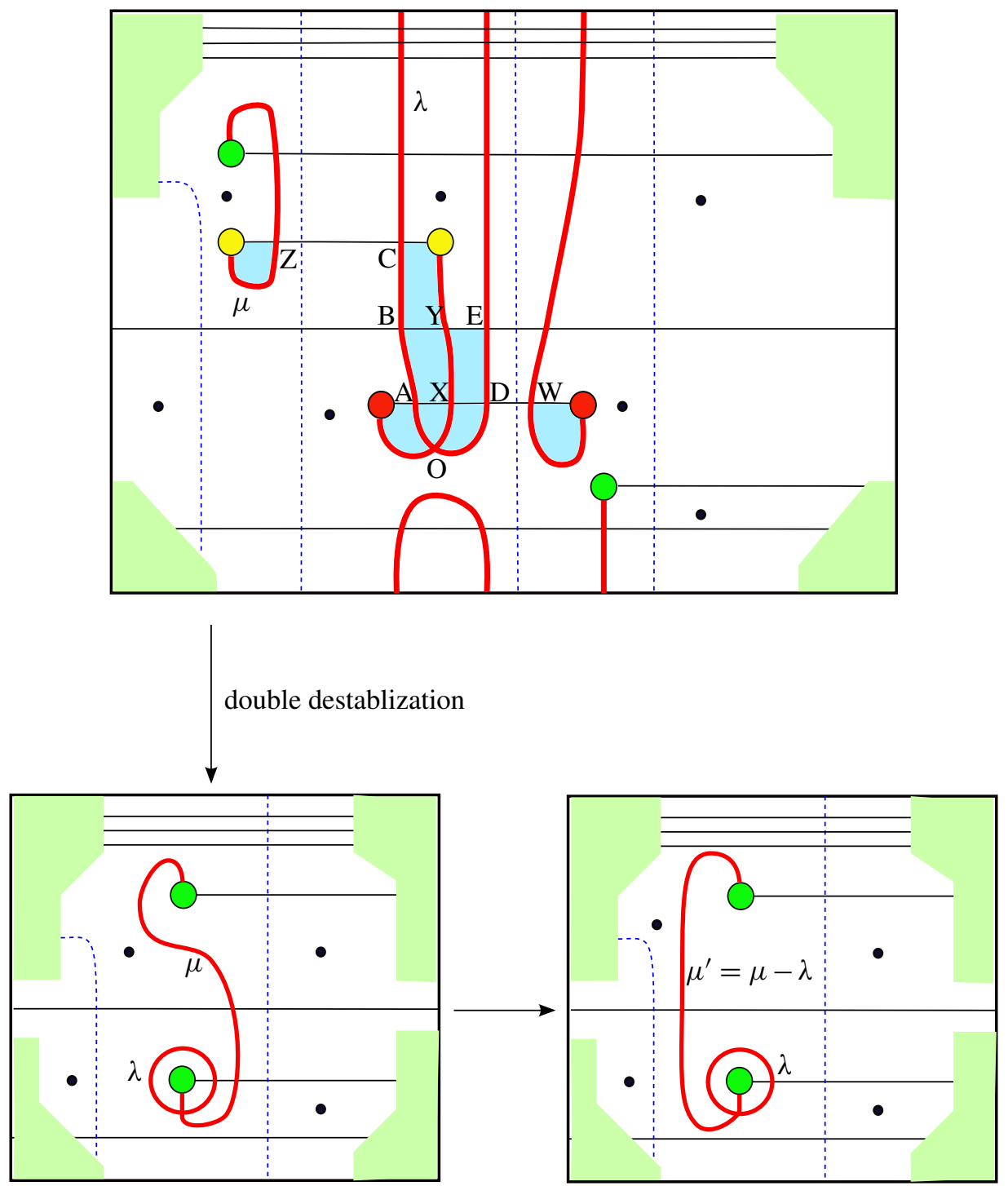

Figure 3: Special Heegaard diagrams for knot complements are the union of the genus- 4 surface $\Sigma_{1}$ with boundary illustrated as the white part of the figure on top with another surface with boundary. The curves $\lambda$ and $\mu$ are illustrated as bold red curves, while $\boldsymbol{\alpha} \cap \Sigma_{1}$ and $\hat{\boldsymbol{\beta}} \cap \Sigma_{1}$ are denoted by black curves and dashed blue curves, respectively. The intersection of $\mu$ and $\lambda$ is denoted by $O$ and some of the intersection points in $\alpha \cap(\lambda \cup \mu)$ are labelled (by $A, B, C, D, E, X, Y, Z$ and $W$ ). Double destablization and a change in the framing (equivalently, in the parametrization of the boundary torus) gives the two Heegaard diagrams on the bottom of the figure. 
Proof Destabilization on $\Sigma_{1}$ gives the equivalent Heegaard diagram, which locally looks like the surface on the lower left part of Figure 3. Changing $\mu$ to $\mu^{\prime}=\mu-\lambda$ in the aforementioned diagram corresponds to changing the parametrization of the boundary. It is thus enough to show that every bordered three-manifold with torus boundary admits a nice Heegaard diagram which locally looks like the lower right side of Figure 3, so that every domain which meets the green region is either a bigon, a rectangle or contains the puncture. If this is the case, every domain in the Heegaard diagram illustrated on the upper side of Figure 3 is either a bigon, a triangle, a rectangle or contains the puncture. In other words, the diagram on the upper side of Figure 3 is nice.

Start with a nice bordered Heegaard diagram for $Y \backslash \operatorname{nd}(K)$ with parametrization given by $\mu^{\prime}$ and $\lambda$, which exists by [6, Proposition 8.2]. Denote the intersection point of $\mu^{\prime}$ and $\lambda$ by $O$. Three of the four quadrants around $O$ are triangles, while the last quadrant contains the marked point $z$. There is thus some curve $\alpha_{i}$ in $\boldsymbol{\alpha}$ which cuts $\mu^{\prime}$ in the points $D$ and $A$ close to $O$ and the curve $\lambda$ in $X$ and $W$ (close to $O$ ), so that the picture around $O$ on $\Sigma$ is the one illustrated in part (a) of Figure 4. We may assume for simplicity that $i=g$. The three triangles are thus $[D O X],[X O A]$ and $[A O W]$. There is a path $\gamma$ disjoint from $\boldsymbol{\beta}_{0} \cup\left\{\mu^{\prime}, \lambda\right\}$ which starts from the interior of the triangle $[A O W]$ and ends at the marked point $z$ and passes only through the rectangles. One may add a 1 -handle to $\Sigma$ with attaching circles placed at the endpoints of $\gamma$. The core of this 1-handle may be added to $\alpha$ as the curve $\alpha_{g+1}$ and the arc $\gamma$ may be completed to a simple closed curve $\beta_{g}$ by attaching its endpoints with an arc going over the 1-handle. This gives a stabilization of the previous Heegaard diagram. We may then handle slide $\alpha_{g+1}$ over $\alpha_{g}$ to obtain the Heegaard diagram illustrated in part (b) of Figure 4.

Next, we may add a 1-handle to the Heegaard diagram with attaching circles placed in the middle of the arcs $[O X]$ and $[O W]$. Denote the arc connecting the above two midpoints by $\delta$. The curve $\mu^{\prime}$ will be renamed $\beta_{g+1}$, the core of this handle will be replaced for $\mu^{\prime}$, the curve $\lambda$ will be modified by deleting the arc $\delta$ from it and replacing a corresponding arc which travels over the 1-handle, and, finally, the arc $\delta$ is completed to a simple closed curve $\alpha_{g+2}$ using the 1-handle. The new Heegaard diagram is illustrated in part (c) of Figure 4. This new Heegaard diagram corresponds to the same bordered three-manifold.

Next, we attach another 1-handle to the Heegaard diagram. The attaching circles are placed on $\lambda$ on the two sides of the arc bounded between the intersection of $\alpha_{g+1}$ and $\lambda$ and the intersection of $\mu^{\prime}$ and $\lambda$. The aforementioned arc may be completed (by adding to it a segment which travels over the 1-handle) to a simple closed curve, which will be replaced for $\lambda$. The remainder of (the old) $\lambda$ may also be completed (again by 


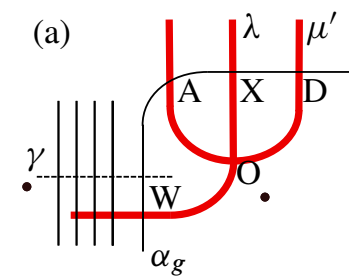

(d)

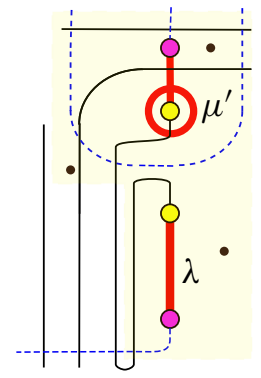

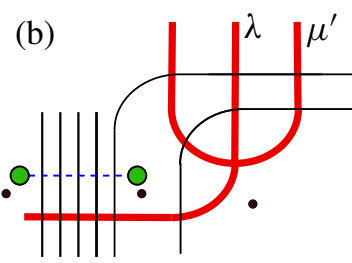

(e)

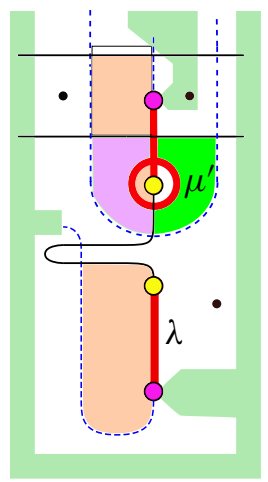

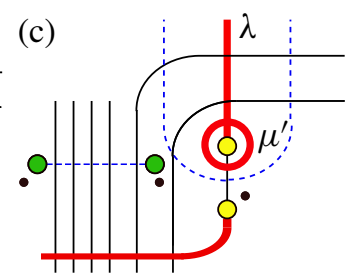

(f)

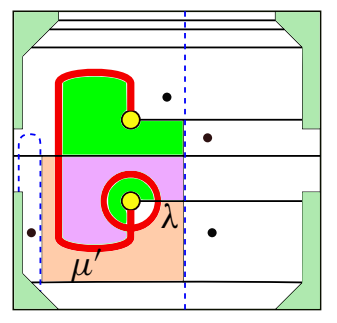

Figure 4: The $\alpha$-curves are denoted by solid black lines, the $\beta$-curves are the dashed blue lines, and the curves $\mu^{\prime}$ and $\lambda$ are denoted by bold red lines. $D_{z}$ is the domain containing bold circles. (a) In a nice Heegaard diagram, three of the quadrants around $O=\mu^{\prime} \cap \lambda$ are triangles. Use an arc $\gamma$ disjoint from $\hat{\boldsymbol{\beta}} \cup\left\{\mu^{\prime}, \lambda\right\}$ to connect the triangle $[A O W]$ to $z$. The closest $\alpha$-curve to $O$ is $\alpha_{g}$. (b) Attach a handle at the endpoints of $\gamma$, complete $\gamma$ to a $\beta$-curve and slide the core of the handle over $\alpha_{g}$ to produce a new $\alpha$-curve. (c) Attach a handle on $\lambda$ at the two sides of $O$ (the attaching circles are painted yellow). Rename $\mu^{\prime}$ to $\beta_{g+1}$ and replace the core of the handle for $\mu^{\prime}$. Push $\lambda$ above the handle and complete the segment on $\mu^{\prime}$ containing $O$ to $\alpha_{g+2}$. (d) Attach a handle on $\lambda$ at the points illustrated by purple circles. The arcs on $\lambda$ connecting the purple attaching circles to the yellow attaching circles may be completed to a closed curve, which will be replaced for $\lambda$. The complement of these two arcs on initial $\lambda$ may be completed to a $\beta$-curve. The core of the 1-handle slides over $\alpha_{g}$ to produce the new $\alpha$-curve. Finally, a finger move modifies $\alpha_{g+2}$. (e)-(f) Re-draw the subsurface of genus 2 around the intersection of $\mu^{\prime}$ and $\lambda$ which was shaded in part (d).

adding to it a segment which travels over the 1-handle) to a simple closed curve, which will be denoted by $\beta_{g+2}$. One may slide the core of the new 1 -handle over $\alpha_{g+1}$ to obtain $\alpha_{g+3}$. Finally, we apply a finger move isotopy to $\alpha_{g+2}$ to create a pair of intersection points between $\alpha_{g+2}$ and $\beta_{g+2}$. The new Heegaard diagram (which still corresponds to the same bordered three-manifold) is illustrated in part (d) of Figure 4 
and a subset of the diagram which lives on a subsurface of genus 2 is re-drawn in part (e) of the same picture, where a 7-gon and a pair of pentagons are painted orange, green and purple, respectively. One may then identify the aforementioned subsurface of genus 2 with the surface illustrated in part (f). To illustrate the correspondence, the domains corresponding to the 7-gon and the two pentagons are painted in the new picture with the relevant colour. This completes the proof of the lemma.

Definition 4.2 For every knot $K \subset Y$ and every framing $\lambda$ for $K$, the Heegaard diagrams of the type constructed in Lemma 4.1 are called special Heegaard diagrams.

\subsection{A combinatorial description of $\mathfrak{f}_{\bullet}(K)$ and $\overline{\mathfrak{f}}_{\bullet}(K)$}

Suppose that $(Y, K)$ denotes a knot $K$ inside a homology sphere $Y$. Let us assume that

$$
\left(\Sigma, \boldsymbol{\alpha}=\left\{\alpha_{1}, \ldots, \alpha_{g}\right\}, \widehat{\boldsymbol{\beta}}=\left\{\beta_{1}, \ldots, \beta_{g-1}\right\}, \mu, \lambda, z\right)
$$

is a special Heegaard diagram for the bordered three-manifold determined by a zeroframed longitude for $K$ inside $Y$. The picture around the intersection point $O$ of the simple closed curves $\mu$ and $\lambda$ is illustrated on the top of Figure 3.

We introduce three auxiliary curves, denoted by $\lambda_{\infty}, \lambda_{0}$ and $\lambda_{1}$, respectively, as in the Heegaard diagram illustrated in Figure 5. The Heegaard diagrams

$$
H_{\bullet}=\left(\Sigma, \boldsymbol{\alpha}, \hat{\boldsymbol{\beta}} \cup\left\{\lambda_{\bullet}\right\} ; u, v, w\right) \quad \text { and } \quad \bar{H}_{\bullet}=\left(\Sigma, \boldsymbol{\alpha}, \hat{\boldsymbol{\beta}} \cup\left\{\lambda_{\bullet}\right\} ; \bar{u}, \bar{v}, \bar{w}\right)
$$

are (triply punctured) diagrams that correspond to the knot $K_{\bullet} \subset Y_{\bullet}(K)$ for $\bullet \in\{0,1, \infty\}$ (note that two of the three punctures are placed in the same connected component of $\Sigma \backslash\left(\boldsymbol{\alpha} \cup \hat{\boldsymbol{\beta}} \cup \lambda_{\bullet}\right)$ for $\left.\bullet \in\{0,1, \infty\}\right)$. The above claim is checked by computing the intersection numbers of each $\lambda$. with the simple closed curves $\mu$ and $\lambda$, since the curves are disjoint from $\hat{\boldsymbol{\beta}}$. Each pair of these three curves intersect each other exactly once. Each of the three diagrams $H_{\bullet}, \bar{H}_{\bullet}, \bullet \in\{0,1, \infty\}$, is a nice Heegaard diagram and they determine the chain complexes $C_{\bullet}=\widehat{\mathrm{CF}}\left(H_{\bullet}\right)=\widehat{\mathrm{CF}}\left(\bar{H}_{\bullet}\right)$. Denote the differential of the complex $C_{\bullet}$ by $d_{\bullet}$ for $\bullet \in\{0,1, \infty\}$. The chain maps $\mathfrak{f}_{\bullet}(K)$ and $\overline{\mathfrak{f}}_{\bullet}(K)$ have a simple combinatorial description, which is discussed in the remainder of this section.

Fix the labelling of the intersection points of $\lambda_{0}, \lambda_{1}, \lambda_{\infty}, \beta_{g-1}$ and $\beta_{g-2}$ with the curves in $\boldsymbol{\alpha}$ as in Figure 5. Let

$$
\left\{P_{0}\right\}=\lambda_{1} \cap \lambda_{\infty}, \quad\left\{P_{1}\right\}=\lambda_{0} \cap \lambda_{\infty} \quad \text { and } \quad\left\{P_{\infty}\right\}=\lambda_{0} \cap \lambda_{1} .
$$

The Heegaard triple

$$
\left(\Sigma, \boldsymbol{\alpha}, \hat{\boldsymbol{\beta}} \cup\left\{\lambda_{1}\right\}, \hat{\boldsymbol{\beta}} \cup\left\{\lambda_{\infty}\right\} ; \bar{u}, \bar{v}, \bar{w}\right)
$$




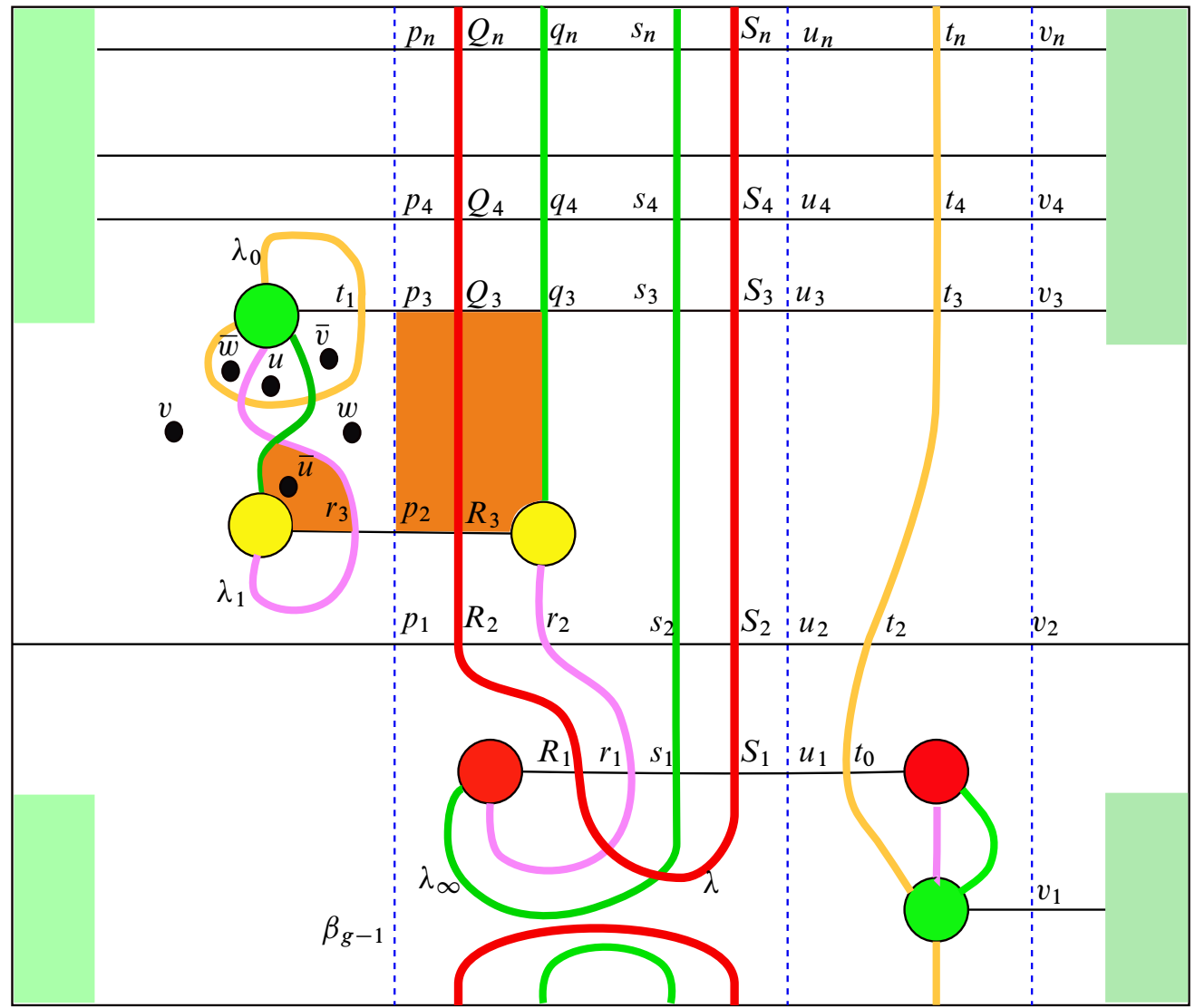

Figure 5: The curves in $\alpha$ are denoted by solid black lines while the curves in $\widehat{\boldsymbol{\beta}}$ are denoted by dashed blue lines. Three simple closed curves $\lambda_{0}, \lambda_{1}$ and $\lambda_{\infty}$ are denoted by bold red, purple and green lines, respectively. Six marked points $u, v, w, \bar{u}, \bar{v}$ and $\bar{w}$ are introduced close to the intersection points of these three curves. The intersection points on $\beta_{g-1}, \lambda_{\infty}$ and $\lambda_{1}$ are labelled. Associated with $i \geq 3$ there is a pentagon with vertices at $P_{0}$, $r_{3}, p_{2}, p_{i}$ and $q_{i}$. For $i=3$ the pentagon is shaded orange in the picture.

determines a combinatorial triangle map $\bar{f}_{0}: C_{1} \rightarrow C_{\infty}$ as follows. Let $\beta_{g-1}$ be the $\beta$-curve which contains the intersection points $p_{1}, p_{2}, \ldots, p_{n}$ in Figure 5. Let $\boldsymbol{x}=\left(x_{1}, \ldots, x_{g}\right)$ be a generator of $C_{1}$ with $x_{i} \in \alpha_{\sigma(i)} \cap \beta_{i}$ for some $\sigma \in S_{g}$, $i=1, \ldots, g-1$, and $x_{g} \in \lambda_{1}$. Define

$$
\bar{f}_{0}(\boldsymbol{x}):= \begin{cases}\left(x_{1}, \ldots, x_{g-1}, s_{i}\right) & \text { if } x_{g}=r_{i}, i=1,2, \\ 0 & \text { otherwise. }\end{cases}
$$


Similarly, the Heegaard triple

$$
\left(\Sigma, \boldsymbol{\alpha}, \widehat{\boldsymbol{\beta}} \cup\left\{\lambda_{1}\right\}, \hat{\boldsymbol{\beta}} \cup\left\{\lambda_{\infty}\right\} ; u, v, w\right)
$$

determines a combinatorial triangle map $f_{0}: C_{1} \rightarrow C_{\infty}$ defined by

$$
f_{0}(\boldsymbol{x}):= \begin{cases}\left(x_{1}, \ldots, x_{g-2}, p_{2}, q_{i}\right) & \text { if }\left(x_{g-1}, x_{g}\right)=\left(p_{i}, r_{3}\right), i \geq 3, \\ \left(x_{1}, \ldots, x_{g-2}, p_{1}, q_{i}\right) & \text { if }\left(x_{g-1}, x_{g}\right)=\left(p_{i}, r_{2}\right), i \geq 3, \\ 0 & \text { otherwise }\end{cases}
$$

The Heegaard triples

$$
\left(\Sigma, \boldsymbol{\alpha}, \widehat{\boldsymbol{\beta}} \cup\left\{\lambda_{0}\right\}, \widehat{\boldsymbol{\beta}} \cup\left\{\lambda_{1}\right\} ; u, v, w\right) \quad \text { and } \quad\left(\Sigma, \boldsymbol{\alpha}, \widehat{\boldsymbol{\beta}} \cup\left\{\lambda_{0}\right\}, \widehat{\boldsymbol{\beta}} \cup\left\{\lambda_{1}\right\} ; \bar{u}, \bar{v}, \bar{w}\right),
$$

correspond to the combinatorial triangle maps $f_{\infty}, \bar{f}_{\infty}: C_{0} \rightarrow C_{1}$. For a generator $\boldsymbol{x}=\left(x_{1}, \ldots, x_{g}\right)$, these two maps are defined by setting

$$
\begin{aligned}
& \bar{f}_{\infty}(\boldsymbol{x})= \begin{cases}\left(x_{1}, \ldots, x_{g-1}, r_{1}\right) & \text { if } x_{g}=t_{0}, \\
0 & \text { otherwise, }\end{cases} \\
& f_{\infty}(\boldsymbol{x})= \begin{cases}\left(x_{1}, \ldots, x_{g-2}, p_{3}, r_{3}\right) & \text { if }\left(x_{g-1}, x_{g}\right)=\left(p_{2}, t_{1}\right), \\
0 & \text { otherwise }\end{cases}
\end{aligned}
$$

Lemma 4.3 With the above notation fixed, $f_{0} \circ f_{\infty}=\bar{f}_{0} \circ \bar{f}_{\infty}=0$.

Proof This is trivial from the combinatorial definitions of $f_{0}, \bar{f}_{0}, f_{\infty}$ and $\bar{f}_{\infty}$.

Let

$$
\Sigma \backslash\left(\boldsymbol{\alpha} \cup \widehat{\boldsymbol{\beta}} \cup \lambda_{0} \cup \lambda_{1}\right)=\left(\coprod_{i=1}^{N} D_{i}\right) \cup D_{\bar{u}} \cup D_{\bar{v}} \cup D_{\bar{w}},
$$

where $D_{\bullet}$ are the regions in the complement of these curves, with $D_{\bar{u}}, D_{\bar{v}}$ and $D_{\bar{w}}$ the regions containing the marked points $\bar{u}, \bar{v}$ and $\bar{w}$, respectively. We set

$$
\beta_{i}^{0}=\beta_{i}, \quad i=1, \ldots, g-1, \quad \text { and } \quad \boldsymbol{\beta}=\left\{\beta_{1}, \ldots, \beta_{g}\right\}=\hat{\boldsymbol{\beta}} \cup\left\{\lambda_{0}\right\} .
$$

The construction of the Heegaard diagram implies the following properties:

- The regions $D_{2}, \ldots, D_{N}$ are rectangles or bigons, while $D_{1}$ is a pentagon.

- One of the corners of the pentagon $D_{1}$ is the unique intersection point $P=$ $P_{\infty}=\lambda_{0} \cap \lambda_{1}$, and the three punctures $\bar{u}, \bar{v}$ and $\bar{w}$ are placed on three of the quadrants around $P$ (other than the quadrant corresponding to $D_{1}$ ).

- All the neighbours of $D_{1}$ (the regions having an edge in common with $D_{1}$ ) are punctured.

- Each $\beta$-curve is adjacent to at least one of the punctured domains. 

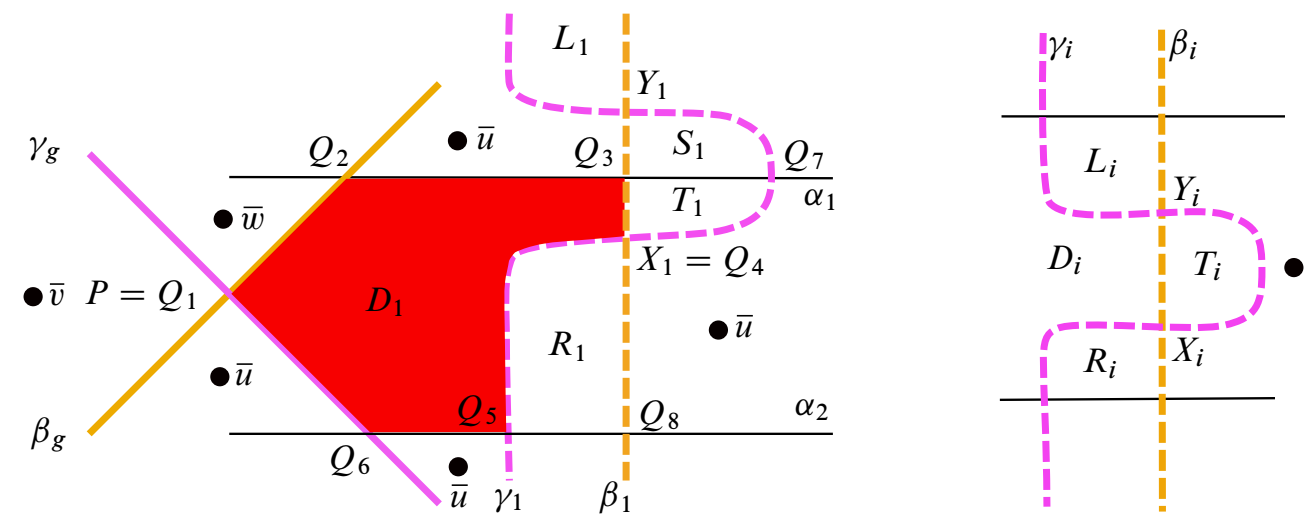

Figure 6: The region around the pentagon $D_{1}$ is illustrated on the left-hand side. The punctured domains are marked by solid circles inside them. The curves in $\boldsymbol{\beta}=\boldsymbol{\beta}_{0}, \boldsymbol{\gamma}=\boldsymbol{\beta}_{1}$ and $\boldsymbol{\alpha}$ have colours orange, pink and black, respectively. The pentagon is changed to a hexagon in the new Heegaard diagram, which is coloured red. The initial pentagon is the union of the hexagon $D_{1}$ with the triangle $R_{1}$. The right-hand side illustrates the labelling near the intersection of $\beta_{i}$ with its Hamiltonian isotope $\gamma_{i}$.

The edges of the pentagon are five arcs: two of them are on $\lambda_{0}$ and $\lambda_{1}$, two of them are on the $\alpha$-curves and one of them is on a $\beta$-curve, which is assumed to be $\beta_{1}$. The $\alpha$-curve which cuts $\lambda_{0}$ in a corner of the pentagon is assumed to be $\alpha_{1}$ and the other one is assumed to be $\alpha_{2}$. Denote the vertices of the pentagon by $P=Q_{1}, Q_{2}$, $Q_{3}, Q_{8}$ and $Q_{6}$ in counter-clockwise order, so that $Q_{1}$ is the intersection point of $\lambda_{0}$ and $\lambda_{1}, Q_{2}$ is on the intersection of $\alpha_{1}$ with $\lambda_{0}$, and $Q_{6}$ is the intersection point of $\lambda_{1}$ with $\alpha_{2}$.

For $i=2, \ldots, g-1$, let $\beta_{i}^{1}=\gamma_{i}$ be a parallel copy of $\beta_{i}$ which is drawn very close to $\beta_{i}$ and is slightly pushed to one of the punctured domains adjacent to $\beta_{i}$ by a finger move, so that a pair of intersection points (denoted by $X_{i}$ and $Y_{i}$ ) is created between these two curves (see the right-hand side picture in Figure 6). Let us assume that the small positively oriented disk connecting these two intersection points (with $\beta_{i}$ on the left and $\gamma_{i}$ on the right) goes from $X_{i}$ to $Y_{i}$. In order to define $\gamma_{1}$, choose a parallel copy of $\beta_{1}$ and push it slightly over the intersection point of $\beta_{1}$ with $\alpha_{1}$ to obtain $\gamma_{1}$, so that a pair of cancelling intersection points $X_{1}$ and $Y_{1}$ is created between $\gamma_{1}$ and $\beta_{1}$ on the two sides of the intersection point $Q_{3}$ of $\alpha_{1}$ and $\beta_{1}$, and so that $\gamma_{1}$ slightly enters the punctured domain next to the $\beta$-edge of the pentagon. The local picture around $D_{1}$ looks like Figure 6, where this procedure is pictured. Let $\gamma_{g}$ be the curve $\lambda_{1}$ and set $\boldsymbol{\beta}_{1}=\boldsymbol{\gamma}=\left\{\gamma_{1}, \ldots, \gamma_{g}\right\}$. 


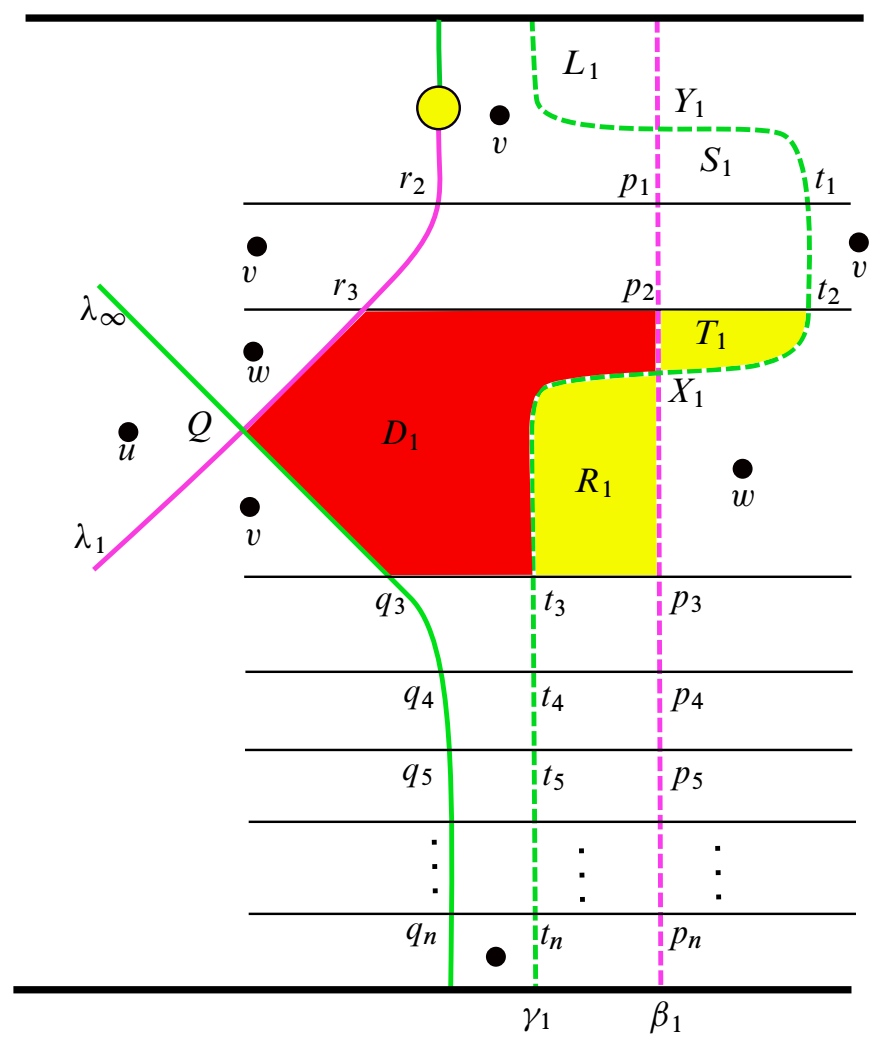

Figure 7: The region around the hexagon $D_{1}$ is illustrated. The labelling of the intersection points in the Heegaard diagram, as well as the labelling of some of the connected components in the complement of the curves, is illustrated. The curves in $\boldsymbol{\beta}_{1}, \boldsymbol{\beta}_{\infty}$ and $\boldsymbol{\alpha}$ have colours pink, green and black, respectively.

In order to construct $\beta_{i}^{\infty}$ for $i=2, \ldots, g-1$, choose a parallel copy of $\gamma^{i}=\beta_{i}^{1}$ and, as this parallel copy enters the bigon $T_{i}$, push it into the neighbouring punctured domain by a finger move. The curve $\beta_{1}^{\infty}$ is constructed as illustrated in Figure 7. We set

$$
\boldsymbol{\beta}_{\infty}=\left\{\beta_{1}^{\infty}, \ldots, \beta_{g-1}^{\infty}, \lambda_{\infty}\right\}
$$

Lemma 4.4 The punctured Heegaard diagrams

$$
\left(\Sigma, \boldsymbol{\alpha}, \boldsymbol{\beta}_{\star}, \boldsymbol{\beta}_{\bullet} ; u, v, w\right) \quad \text { and } \quad\left(\Sigma, \boldsymbol{\alpha}, \boldsymbol{\beta}_{\star}, \boldsymbol{\beta}_{\bullet} ; \bar{u}, \bar{v}, \bar{w}\right)
$$

for $(\star, \bullet) \in\{(0,1),(1, \infty)\}$ do not contain any non-trivial, positive, triply periodic domains. 
Proof Let $\mathcal{D}$ denote a positive, triply periodic domain in the Heegaard diagram $\left(\Sigma, \boldsymbol{\alpha}, \boldsymbol{\beta}_{1}, \boldsymbol{\beta}_{\infty} ; u, v, w\right)$. Thus,

$$
\partial \mathcal{D}=\sum_{i=1}^{g} a_{i} \alpha_{i}+\sum_{i=1}^{g-1} b_{i} \beta_{i}^{1}+\sum_{i=1}^{g-1} c_{i} \beta_{i}^{\infty}+b \lambda_{1}+c \lambda_{\infty} .
$$

Let $\mathcal{D}_{i}$ denote the doubly periodic domain with $\partial \mathcal{D}_{i}=\beta_{i}^{1}-\beta_{i}^{\infty}$ for $i=1, \ldots, g-1$. Setting $\mathcal{D}^{\prime}=\mathcal{D}-\sum_{i=1}^{g-1} b_{i} \mathcal{D}_{i}$, we find

$$
\partial \mathcal{D}^{\prime}=\sum_{i=1}^{g} a_{i} \alpha_{i}+\sum_{i=1}^{g-1}\left(c_{i}-b_{i}\right) \beta_{i}^{\infty}+b \lambda_{1}+c \lambda_{\infty} .
$$

Since the left-hand side is trivial in $H_{1}(Y \backslash \operatorname{nd}(K) ; \mathbb{Z})$, so is the right-hand side. This implies that $c=-b$. Let $\mathcal{D}_{0}$ denote the triply periodic domain in the punctured Heegaard triple $\left(\Sigma, \boldsymbol{\beta}_{0}, \boldsymbol{\beta}_{1}, \boldsymbol{\beta}_{\infty} ; u, v, w\right)$ with $\partial \mathcal{D}_{0}=\lambda_{1}-\lambda_{0}-\lambda_{\infty}$. For $\mathcal{D}^{\prime \prime}=\mathcal{D}^{\prime}-b \mathcal{D}_{0}$ we thus obtain

$$
\partial \mathcal{D}^{\prime \prime}=\sum_{i=1}^{g} a_{i} \alpha_{i}+\sum_{i=1}^{g-1}\left(c_{i}-b_{i}\right) \beta_{i}^{\infty}+b \lambda_{0} .
$$

In other words, $\mathcal{D}^{\prime \prime}$ is a doubly periodic domain for the nice (and hence weakly admissible) Heegaard diagram

$$
\left(\Sigma, \boldsymbol{\alpha}, \boldsymbol{\beta}_{\infty} \cup\left\{\lambda_{0}\right\} \backslash\left\{\lambda_{\infty}\right\} ; u, v, w\right) .
$$

The coefficients of $\mathcal{D}^{\prime \prime}$ and all $\mathcal{D}_{i}, i=1, \ldots, g-1$, over the small triangle bounded between $\lambda_{0}, \lambda_{1}$ and $\lambda_{\infty}$ is zero. In other words, the coefficient of

$$
\mathcal{D}=\mathcal{D}^{\prime \prime}+b \mathcal{D}_{0}+\sum_{i=1}^{g-1} b_{i} \mathcal{D}_{i}
$$

over this small triangle is $b$, which should thus be non-negative. Choosing this triangle sufficiently small we may thus assume that the total area of $b \mathcal{D}_{0}$ is negative unless $b=0$.

One may choose the area form on the surface $\Sigma$ so that all doubly periodic domains for the punctured Heegaard diagram $\left(\Sigma, \boldsymbol{\alpha}, \boldsymbol{\beta}_{\infty} \cup\left\{\lambda_{0}\right\} \backslash\left\{\lambda_{\infty}\right\} ; u, v, w\right)$ and all $\mathcal{D}_{i}$, $i=1, \ldots, g-1$, have zero total area. However, this implies that the total area of $\mathcal{D}$ is the same as the total area of $b \mathcal{D}_{0}$, which is at most zero. Since $\mathcal{D}$ is a positive domain, we conclude $\mathcal{D}=0$. This completes the proof for the triple $\left(\Sigma, \boldsymbol{\alpha}, \boldsymbol{\beta}_{1}, \boldsymbol{\beta}_{\infty} ; u, v, w\right)$. The proof for the other triples is completely similar.

The Heegaard diagrams

$$
\left(\Sigma, \boldsymbol{\alpha}, \boldsymbol{\beta}_{\bullet} ; u, v, w\right) \quad \text { and } \quad\left(\Sigma, \boldsymbol{\alpha}, \boldsymbol{\beta}_{\bullet} ; \bar{u}, \bar{v}, \bar{w}\right)
$$


are nice so, by Sarkar and Wang [16], the differentials of the complexes

$$
\widehat{\mathrm{CF}}\left(\Sigma, \boldsymbol{\alpha}, \boldsymbol{\beta}_{\bullet} ; u, v, w\right) \text { and } \widehat{\mathrm{CF}}(\Sigma, \boldsymbol{\alpha}, \boldsymbol{\beta} \bullet ; \bar{u}, \bar{v}, \bar{w})
$$

are given by counts of bigons and rectangles.

Theorem 4.5 Under the above identification of the chain complexes $\left(C_{\bullet}, d_{\bullet}\right)$,

$$
\begin{aligned}
f_{0} & =\phi\left(\Sigma, \boldsymbol{\alpha}, \boldsymbol{\beta}_{1}, \boldsymbol{\beta}_{\infty} ; u, v, w\right), \quad \bar{f}_{0}=\phi\left(\Sigma, \boldsymbol{\alpha}, \boldsymbol{\beta}_{1}, \boldsymbol{\beta}_{\infty} ; \bar{u}, \bar{v}, \bar{w}\right), \\
f_{\infty} & =\phi\left(\Sigma, \boldsymbol{\alpha}, \boldsymbol{\beta}_{0}, \boldsymbol{\beta}_{1} ; u, v, w\right), \quad \bar{f}_{\infty}=\phi\left(\Sigma, \boldsymbol{\alpha}, \boldsymbol{\beta}_{0}, \boldsymbol{\beta}_{1} ; \bar{u}, \bar{v}, \bar{w}\right) .
\end{aligned}
$$

\subsection{Proof of Theorem 4.5}

A similar discussion is carried over in [3] (and in particular Theorem 2.3 from that paper). We repeat the proof, in most parts with more details, to keep the paper easier to read.

Proof We start by proving the statement for $\bar{f}_{\infty}$. Note that the top generator $\Theta$ of the Heegaard Floer homology group $\widehat{\mathrm{HF}}\left(\#^{g-1} S^{1} \times S^{2}\right)$ coming from the Heegaard diagram $(\Sigma, \boldsymbol{\beta}, \boldsymbol{\gamma} ; \bar{u}, \bar{v}, \bar{w})$ is the generator $\left\{P, X_{1}, \ldots, X_{g-1}\right\}$.

Let $\boldsymbol{x}=\left(x_{1}, \ldots, x_{g}\right)$ and $\boldsymbol{y}=\left(y_{1}, \ldots, y_{g}\right)$ be generators with $x_{i} \in \alpha_{\sigma(i)} \cap \beta_{i}$ and $y_{i} \in \alpha_{\tau(i)} \cap \gamma_{i}$, with $\sigma, \tau \in S_{g}$. Let $\Delta: \mathbb{D} \rightarrow \operatorname{Sym}^{g}(\Sigma)$ be the homotopy class of a triangle in $\pi_{2}(\boldsymbol{x}, \Theta, \boldsymbol{y})$, with Maslov index zero, so that it supports a holomorphic representative and remains disjoint from the punctures.

There are two types of domain in the complement $\Sigma \backslash(\boldsymbol{\alpha} \cup \boldsymbol{\beta} \cup \boldsymbol{\gamma})$ of the curves, the large domains and the small domains. The small domains are those created between the parallel pairs of curves $\gamma_{i}$ and $\beta_{i}(i=1, \ldots, g-1)$, and their area may be chosen arbitrarily small by choosing $\gamma_{i}$ close enough to $\beta_{i}$. The large domains are the rest of the domains, which are in correspondence with the domains $D_{\bullet}, \bullet \in\{\bar{u}, \bar{v}, \bar{w}, 1, \ldots, N\}$, introduced above. We abuse the notation and still denote these new regions by $D_{\text {. }}$.

Let us assume that the small bigon connecting $X_{i}$ to $Y_{i}$ is denoted by $T_{i}$ and the region having the small interval $\left[X_{i}, Y_{i}\right]$ on $\beta_{i}$ in common with $T_{i}$ is $D_{i}, i=2, \ldots, g-1$. Then there are two triangles with corners $X_{i}$ and $Y_{i}$ that have an edge in common with $D_{i}$, which will be denoted by $R_{i}$ and $L_{i}$, respectively. For $i=1$, instead of these three regions we have four triangles with one corner being $X_{1}$ or $Y_{1}$, which will be denoted by $R_{1}, T_{1}, S_{1}$ and $L_{1}$, respectively (as they appear while we travel on $\beta_{1}$ from $X_{1}$ to $Y_{1}$; see Figure 6). We are implicitly assuming that the regions $D_{i}$ for $i=1, \ldots, g-1$ (as described above) are different, while it may happen that this is not the case. However, the argument we give below remains true in general and only needs notational corrections. 
Let $\mathcal{D}=\mathcal{D}(\Delta)$ denote the domain (ie the 2-chain on $\Sigma$ ) associated with the triangle class $\Delta$. Let $d_{i} \geq 0$ denote the coefficient of $D_{i}$ in $\mathcal{D}$. Similarly, denote the coefficients of $T_{i}, R_{i}$ and $L_{i}$ by $t_{i}, r_{i}$ and $l_{i}$, respectively. The coefficient of $S_{1}$ will be denoted by $s_{1}$. Of course, there are other regions which may appear in $\mathcal{D}$ with positive coefficient, but all such regions are bigons or rectangles. Since $P$ appears in $\Theta$ and three of the corners around $P$ are punctured, the coefficient $d_{1}$ is equal to 1 .

Let $P=Q_{1}, Q_{2}, \ldots, Q_{6}$ denote the corners of $D_{1}$ (now a hexagon) in counterclockwise order (so $Q_{4}=X_{1}$ ). Since two opposite quadrants around each one of $Q_{2}$ and $Q_{6}$ are punctured, we have $x_{g}=Q_{2}$ and $y_{g}=Q_{6}$. Thus, $Q_{3}$ is not one of $x_{1}, \ldots, x_{g}$ and $Q_{5}$ is not one of $y_{1}, \ldots, y_{g}$. Considering the local coefficients around $Q_{3}$, we conclude that $t_{1}=1+s_{1}$. If $Q_{7}$ is the third corner of $T_{1}$ (other than $Q_{3}$ and $Q_{4}$ ), in order for $\mathcal{D}$ to be a non-negative domain we need $x_{1}=Q_{7}$ and the 4 local coefficients around $Q_{7}$ are forced to be $t_{1}=1+s_{1}, s_{1}, 0$ and 0 in the counter-clockwise order. Two opposite quadrants around $Y_{1}$ have zero coefficients in $\mathcal{D}$. Since $Y_{1}$ does not appear in $\Theta$, this implies that $s_{1}=l_{1}=0$ (thus $t_{1}=1$ ). Similarly, considering the local coefficients around $p_{1}$ we conclude $r_{1}=1$. Since $Q_{5}$ is not among $y_{1}, \ldots, y_{g}$, the local coefficients around $Q_{5}$ are $1, r_{1}=1,0$ and 0 in the counter-clockwise order. Let $Q_{8}$ be the third corner of $R_{1}$ other than $Q_{4}$ and $Q_{5}$. Since two opposite corners around $Q_{8}$ have zero coefficient and $r_{1}=1$, we have $x_{1}=Q_{8}$. Thus $\mathcal{D}=\mathcal{D}^{\prime}+\mathcal{D}_{1}=\mathcal{D}^{\prime}+\left(R_{1}+D_{1}+T_{1}\right)$, where $\mathcal{D}^{\prime}$ is a non-negative 2-chain which is disjoint from $\mathcal{D}_{1}$ and $\mathcal{D}_{1}$ is a hexagon with five acute angles and one obtuse angle and with vertices $\left\{P, y_{g}, x_{1}, X_{1}, y_{1}, x_{g}\right\}$. The contribution of $\mathcal{D}_{1}$ to the index of $\Delta$ is zero, by Sarkar's formula [15].

By Sarkar's formula for the index of triangles [15],

$$
\mu(\Delta)=e(\mathcal{D})+\mu_{\boldsymbol{x}}(\mathcal{D})+\mu_{\boldsymbol{y}}(\mathcal{D})+\mathfrak{b}(\mathcal{D}) \cdot \mathfrak{c}(\mathcal{D})-\frac{1}{2} g
$$

Here $e(\mathcal{D})$ is the Euler measure of the domain $\mathcal{D}, \mathfrak{b}(\mathcal{D})$ is the part of $\partial \mathcal{D}$ on the $\beta-$ curves, and $\mathfrak{c}(\mathcal{D})$ is the part of $\partial \mathcal{D}$ on the $\gamma$-curves. Furthermore, $\mu_{\boldsymbol{x}}(\mathcal{D})$ and $\mu_{\boldsymbol{y}}(\mathcal{D})$ denote the local contributions of the intersection points included in $\boldsymbol{x}$ and $\boldsymbol{y}$, respectively, to the corners of $\mathcal{D}$. We refer to [15] for more detailed definitions. Separating $\mathcal{D}_{1}$ — which has Maslov index 0 - from $\mathcal{D}$ we obtain the equality

$$
\mu(\Delta)=e\left(\mathcal{D}_{s}\right)+e\left(\mathcal{D}_{l}\right)+\mu_{\boldsymbol{x}}\left(\mathcal{D}^{\prime}\right)+\mu_{\boldsymbol{y}}\left(\mathcal{D}^{\prime}\right)+\mathfrak{b}\left(\mathcal{D}^{\prime}\right) \cdot \mathfrak{c}\left(\mathcal{D}^{\prime}\right)-\frac{1}{2}(g-2) .
$$

Here $\mathcal{D}_{s}$ denotes the part of $\mathcal{D}^{\prime}$ which uses the regions $D_{i}, R_{i}, T_{i}$ and $L_{i}$ for $i=2, \ldots, g-1$ and $\mathcal{D}_{l}=\mathcal{D}^{\prime}-\mathcal{D}_{s}$. Clearly, $e\left(\mathcal{D}_{l}\right) \geq 0$ and

$$
\mathcal{D}_{s}=\sum_{i=2}^{g-1}\left(d_{i} D_{i}+t_{i} T_{i}+r_{i} R_{i}+l_{i} L_{i}\right) .
$$


Considering the local coefficients around $X_{i}$ and $Y_{i}$, we conclude $r_{i}=l_{i}+1$ and $d_{i}=t_{i}+l_{i}$. Having in mind that $T_{i}$ are bigons, $R_{i}$ and $L_{i}$ are triangles and $D_{i}$ are hexagons, this implies the following computation:

$$
\begin{aligned}
e\left(\mathcal{D}_{S}\right) & =\sum_{i=2}^{g-1}\left(\left(t_{i}+l_{i}\right) e\left(D_{i}\right)+t_{i} e\left(T_{i}\right)+\left(l_{i}+1\right) e\left(R_{i}\right)+l_{i} e\left(L_{i}\right)\right) \\
& =\sum_{i=2}^{g-1}\left(\left(t_{i}+l_{i}\right)\left(-\frac{1}{2}\right)+t_{i}\left(\frac{1}{2}\right)+\left(l_{i}+1\right)\left(\frac{1}{4}\right)+l_{i}\left(\frac{1}{4}\right)\right)=\frac{1}{4}(g-2) .
\end{aligned}
$$

The 1-chain $\mathfrak{b}\left(\mathcal{D}^{\prime}\right)$ is a union of 1 -chains on $\beta_{i}, i=2, \ldots, g-1$, denoted by $\mathfrak{b}_{i}\left(\mathcal{D}^{\prime}\right)$. Similarly we have $\mathfrak{c}\left(\mathcal{D}^{\prime}\right)=\sum_{i=2}^{g-1} \mathfrak{c}_{i}\left(\mathcal{D}^{\prime}\right)$. It is clear that $\mathfrak{b}_{i}\left(\mathcal{D}^{\prime}\right)$ and $\mathfrak{c}_{j}\left(\mathcal{D}^{\prime}\right)$ are disjoint unless $i=j$. In this latter case, the only possible geometric intersections are at $X_{i}$ and $Y_{i}$, where the intersection numbers are $\left(l_{i}+\frac{1}{2}\right)\left(t_{i}-\frac{1}{2}\right)$ and $-l_{i} t_{i}$, respectively. Thus,

$$
\mathfrak{b}\left(\mathcal{D}^{\prime}\right) \cdot \mathfrak{c}\left(\mathcal{D}^{\prime}\right)=\sum_{i=2}^{g-1}\left(\left(l_{i}+\frac{1}{2}\right)\left(t_{i}-\frac{1}{2}\right)-l_{i} t_{i}\right)=-\frac{1}{4}(g-2)+\frac{1}{2} \sum_{i=2}^{g-1}\left(t_{i}-l_{i}\right) .
$$

Let us now consider the coefficients around the intersection points $x_{i}$ and $y_{i}$ for $i=2, \ldots, g-1$. Since $x_{i}$ is on $\beta_{i}$, there are non-negative integers $a_{i}, b_{i}, c_{i}$ and $e_{i}$ such that the local coefficients around $x_{i}$ are $a_{i}, b_{i}, b_{i}+l_{i}+1$ and $a_{i}+l_{i}$, and the local coefficients around $y_{i}$ are $c_{i}, e_{i}, e_{i}+t_{i}-1$ and $c_{i}+t_{i}$. Thus,

$$
\mu_{\boldsymbol{x}}\left(\mathcal{D}^{\prime}\right)+\mu_{\boldsymbol{y}}\left(\mathcal{D}^{\prime}\right)=\frac{1}{2} \sum_{i=2}^{g-1}\left(\left(a_{i}+b_{i}+c_{i}+e_{i}\right)+\left(l_{i}+t_{i}\right)\right) .
$$

Combining (9), (10) and (11) and replacing for the terms in the definition of $\mu(\Delta)$, we obtain

$$
\begin{aligned}
0=\mu(\Delta) & =e\left(\mathcal{D}_{s}\right)+e\left(\mathcal{D}_{l}\right)+\mu_{\boldsymbol{x}}\left(\mathcal{D}^{\prime}\right)+\mu_{\boldsymbol{y}}\left(\mathcal{D}^{\prime}\right)+\mathfrak{b}\left(\mathcal{D}^{\prime}\right) \cdot \mathfrak{c}\left(\mathcal{D}^{\prime}\right)-\frac{1}{2}(g-2) \\
& =e\left(\mathcal{D}_{l}\right)-\frac{1}{2}(g-2)+\frac{1}{2} \sum_{i=2}^{g-1}\left(a_{i}+b_{i}+c_{i}+e_{i}+2 t_{i}\right) \\
& \geq \frac{1}{2} \sum_{i=2}^{g-1}\left(a_{i}+b_{i}+c_{i}+\left(e_{i}+t_{i}-1\right)+t_{i}\right) .
\end{aligned}
$$

Note that $e_{i}+t_{i}-1$ is the coefficient of one of the domains around $y_{i}$ and is thus non-negative. The above inequality thus implies that $a_{i}=b_{i}=c_{i}=t_{i}=0$ and $e_{i}=1$ for $i=2, \ldots, g-1$. Thus, the coefficients on the two sides of $\gamma_{i}$ either agree or differ by 1 , and the coefficients on the two sides of $\beta_{i}$ differ either by $l_{i}$ or by $l_{i}+1$. If we 
start from $y_{i}$, where on the left (or right) side of $y_{i}$ the coefficients on the two sides of $\gamma_{i}$ are zero, and travel on the $\alpha$ curve intersecting $\gamma_{i}$ (ie orthogonal to $\gamma_{i}$ ) until we get to an intersection point with $\beta_{i}$, as we pass $\beta_{i}$ the coefficient changes either to $-l_{i}$ or to $-l_{i}-1$. Since the latter is negative, the former happens and $l_{i}=0$. It is easy to see from here that $x_{i}$ and $y_{i}$ are the corresponding intersection points of $\beta_{i}$ and $\gamma_{i}$ with the same $\alpha$-curve and that the domain $\mathcal{D}^{\prime}$ is a union of obvious triangles which are disjoint from each other.

We conclude that the domain of $\Delta$ is the disjoint union of $g-2$ simple triangles with a hexagon with five acute angles and one obtuse angle. It is quite well known that the moduli space corresponding to this homotopy class contributes 1 to the triangle map for a generic path of almost complex structures. These are thus the only holomorphic triangles which contribute to the chain map $\bar{f}_{\infty}$ defined using the Heegaard triple $(\Sigma, \boldsymbol{\alpha}, \boldsymbol{\beta}, \boldsymbol{\gamma} ; \bar{u}, \bar{v}, \bar{w})$. Under the obvious identification of $\widehat{\mathrm{CF}}(\Sigma, \boldsymbol{\alpha}, \boldsymbol{\gamma} ; \bar{u}, \bar{v}, \bar{w})$ with $\widehat{\mathrm{CF}}\left(\Sigma, \boldsymbol{\alpha}, \boldsymbol{\beta}_{1} ; \bar{u}, \bar{v}, \bar{w}\right)$, this is just the map which replaces the pair $\left\{Q_{2}, Q_{8}\right\}$ with $\left\{Q_{6}, Q_{7}\right\}$. This completes the proof of Theorem 4.5 for $\bar{f}_{\infty}$.

The proofs of the other three claims are completely similar. In fact, the proofs of the statement of the theorem for $\bar{f}_{0}$ and $f_{\infty}$ are even easier, since the domains which are not punctured in the corresponding Heegaard triple are all bigons, rectangles or triangles. We thus only need to use the second part of the above argument in these two cases (and the study of the neighbourhood of the hexagon is not needed). The proof of the claim for $f_{0}$ requires some more serious modification, which will be outlined below.

Note that the Heegaard triples $\left(\Sigma, \boldsymbol{\alpha}, \boldsymbol{\beta}_{1}, \boldsymbol{\beta}_{\infty} ; u, v, w\right)$ and

$$
\left(\Sigma, \boldsymbol{\alpha}, \boldsymbol{\beta}^{\prime}=\widehat{\boldsymbol{\beta}} \cup\left\{\lambda_{1}\right\}, \boldsymbol{\gamma}^{\prime}=\left\{\beta_{1}^{\infty}, \gamma_{2}, \ldots, \gamma_{g-1}, \lambda_{\infty}\right\} ; u, v, w\right)
$$

may be identified using a diffeomorphism of the surface $\Sigma$. It is thus enough to show that $f_{0}=\phi\left(\Sigma, \boldsymbol{\alpha}, \boldsymbol{\beta}^{\prime}, \boldsymbol{\gamma}^{\prime} ; u, v, w\right)$. This allows us to keep the same labelling for the points $X_{i}, Y_{i}, i=2, \ldots, g-2$. For the intersection points on $\gamma_{1}^{\prime}=\beta_{1}^{\infty}$ and $\beta_{1}$ as well as some of the intersection points on $\lambda_{\infty}$ and $\lambda_{1}$, we use the labelling of Figure 7 . We abuse the notation and denote the two intersection points between $\beta_{1}$ and $\gamma_{1}^{\prime}$ by $X_{1}$ and $Y_{1}$. Moreover, some of the regions in the neighbourhood of $X_{1}$ and $Y_{1}$ are labelled: again by abuse of notation, we denote these regions by $D_{1}, R_{1}, L_{1}, S_{1}$ and $T_{1}$ (see Figure 7). Let us use $d_{i}, r_{i}, s_{i}, t_{i}$ and $l_{i}$ to denote the coefficients of the domains $D_{i}, R_{i}, S_{i}, T_{i}$ and $L_{i}$ in the 2-chain $\mathcal{D}$ associated with a holomorphic triangle connecting $\boldsymbol{x}=\left(x_{1}, \ldots, x_{g}\right), \boldsymbol{y}=\left(y_{1}, \ldots, y_{g}\right)$ and $\Theta$ that contributes to 
$\phi\left(\Sigma, \boldsymbol{\alpha}, \boldsymbol{\beta}^{\prime}, \boldsymbol{\gamma}^{\prime} ; u, v, w\right)$. We assume that, for some elements $\sigma, \tau \in S_{g}$,

$$
\begin{aligned}
& x_{i} \in \begin{cases}\beta_{i} \cap \alpha_{\sigma(i)} & \text { if } i=1, \ldots, g-1, \\
\lambda_{1} \cap \alpha_{\sigma(g)} & \text { if } i=g,\end{cases} \\
& y_{i} \in \begin{cases}\gamma_{i} \cap \alpha_{\tau(i)} & \text { if } i=2, \ldots, g-1, \\
\gamma_{1}^{\prime} \cap \alpha_{\tau(1)} & \text { if } i=1, \\
\lambda_{0} \cap \alpha_{\tau(g)} & \text { if } i=g .\end{cases}
\end{aligned}
$$

The examination of the coefficients in Figure 7 implies the following:

- We have $d_{1}=r_{1}=t_{1}=1$ and $s_{1}=l_{1}=0$.

- Either $x_{g}=r_{2}$ and $y_{1}=t_{1}$, or $x_{g}=r_{3}$ and $y_{1}=t_{2}$.

- There are $j, k \in\{3,4, \ldots, n\}$ such that $y_{g}=q_{k}$ and $x_{1}=p_{j}$.

Let us write $\mathcal{D}=\mathcal{D}_{s}+\mathcal{D}_{l}$, where

$$
\mathcal{D}_{s}:=s_{1} S_{1}+\sum_{i=1}^{g-1}\left(d_{i} D_{i}+t_{i} T_{i}+r_{i} R_{i}+l_{i} L_{i}\right) \quad \text { and } \quad \mathcal{D}_{l}:=\mathcal{D}-\mathcal{D}_{s} .
$$

Considering the local coefficients at $X_{i}$ and $Y_{i}$, we find $r_{i}=l_{i}+1$ and $d_{i}=t_{i}+l_{i}$. Applying the index formula in (8) we obtain

$$
\begin{aligned}
0 & =e(\mathcal{D})+\mu_{\boldsymbol{x}}(\mathcal{D})+\mu_{\boldsymbol{y}}(\mathcal{D})+\mathfrak{b}(\mathcal{D}) \cdot \mathfrak{c}(\mathcal{D})-\frac{1}{2} g \\
& =\left(e\left(\mathcal{D}_{l}\right)+\left(-\frac{1}{2}+\frac{1}{4}+\frac{1}{4}\right)+\frac{1}{4}(g-2)\right)+\mu_{\boldsymbol{x}}(\mathcal{D})+\mu_{\boldsymbol{y}}(\mathcal{D})+\mathfrak{b}(\mathcal{D}) \cdot \mathfrak{c}(\mathcal{D})-\frac{1}{2} g \\
& \geq \mu_{\boldsymbol{x}}(\mathcal{D})+\mu_{\boldsymbol{y}}(\mathcal{D})+\mathfrak{b}(\mathcal{D}) \cdot \mathfrak{c}(\mathcal{D})-\frac{1}{4}(g+2) .
\end{aligned}
$$

The 1-chains $\mathfrak{b}(\mathcal{D})$ and $\mathfrak{c}(\mathcal{D})$ may be written as

$$
\mathfrak{b}(\mathcal{D})=\sum_{i=1}^{g} \mathfrak{b}_{i}(\mathcal{D}) \quad \text { and } \quad \mathfrak{c}(\mathcal{D})=\sum_{i=1}^{g} \mathfrak{c}_{i}(\mathcal{D})
$$

as before. Note that $\mathfrak{b}_{1}(\mathcal{D})$ is the arc on $\beta_{1}$ from $X_{1}$ to $p_{j}$, while $\mathfrak{c}_{1}(\mathcal{D})$ is the arc from one of $t_{1}$ or $t_{2}$ to $X_{1}$. Moreover, $\mathfrak{b}_{g}(\mathcal{D})$ is the arc on $\lambda_{1}$ from $Q$ to one of $r_{2}$ or $r_{3}$, while $\mathfrak{c}_{g}(\mathcal{D})$ is the arc on $\lambda_{\infty}$ from $q_{i}$ to $Q$. Thus,

$$
\mathfrak{b}(\mathcal{D}) \cdot \mathfrak{c}(\mathcal{D})=\left(-\frac{1}{4}+\frac{1}{4}\right)+\sum_{i=2}^{g-1}\left(\left(l_{i}+\frac{1}{2}\right)\left(t_{i}-\frac{1}{2}\right)-l_{i} t_{i}\right)=-\frac{1}{4}(g-2)+\frac{1}{2} \sum_{i=2}^{g-1}\left(t_{i}-l_{i}\right)
$$

Let us now assume that the local coefficients around $x_{i}$ are $a_{i}, b_{i}, b_{i}+l_{i}+1$ and $a_{i}+l_{i}$, while the local coefficients around $y_{i}$ are $c_{i}, e_{i}, e_{i}+t_{i}-1$ and $c_{i}+t_{i}$ for $i=2, \ldots, g-1$. The corresponding local coefficients around $x_{1}, y_{1}, x_{g}$ and $y_{g}$ would 
be $\left(a_{1}, b_{1}, b_{1}+1, a_{1}\right),\left(c_{1}, e_{1}, e_{1}, c_{1}+1\right),(0,0,1,0)$ and $(0,0,0,1)$, respectively, for some non-negative integers $a_{i}, b_{i}, c_{i}$ and $e_{i}, i=1, \ldots, g-1$. Thus,

$$
\mu_{\boldsymbol{x}}(\mathcal{D})+\mu_{\boldsymbol{y}}(\mathcal{D})=\frac{1}{2}+\frac{1}{2} \sum_{i=1}^{g-1}\left(\left(a_{i}+b_{i}+c_{i}+e_{i}\right)+\left(l_{i}+t_{i}\right)\right)
$$

If we combine (12), (13) and (14), we find

$0 \geq-\frac{1}{2} g+\left(\frac{1}{2}\right)+\frac{1}{2} \sum_{i=1}^{g-1}\left(a_{i}+b_{i}+c_{i}+e_{i}+2 t_{i}\right)=\frac{1}{2} \sum_{i=1}^{g-1}\left(a_{i}+b_{i}+c_{i}+\left(e_{i}+t_{i}-1\right)+t_{i}\right)$.

As in the proof of the theorem for $\bar{f}_{\infty}$, this implies that $a_{i}=b_{i}=c_{i}=t_{i}=0$, while $e_{i}=1$ for $i=1, \ldots, g-1$. It is easy to see from here that $j=k$ and complete the proof as before.

\subsection{The maps $\theta(K)$ and $\bar{\theta}(K)$}

Let $\mathbb{H}_{\bullet}$ denote the homology of the chain complex $C$ • for $\bullet \in\{\infty, 1,0\}$. If we choose a representative $a \in C_{0}$ of a class

$$
[a] \in \operatorname{Ker}\left(\left(f_{\infty}\right)_{*}\right) \subset \mathbb{H}_{0},
$$

there exists some $b \in C_{1}$ such that $f_{\infty}(a)=d_{1}(b)$. Then $d_{\infty}\left(f_{0}(b)\right)=f_{0}\left(d_{1}(b)\right)=$ $f_{0}\left(f_{\infty}(a)\right)=0$, so $f_{0}(b)$ is closed and represents a class in $\mathbb{H}_{\infty}$. If we replace $b$ with another element $b^{\prime}=b+\Delta b$ such that $d_{1}\left(b^{\prime}\right)=f_{\infty}(a), \Delta b$ is closed (ie it represents an element in $\left.\mathbb{H}_{1}\right)$. The difference $f_{0}\left(b^{\prime}\right)-f_{0}(b)=f_{0}(\Delta b)$ is an element in $\operatorname{Im}\left(\left(f_{0}\right)_{*}\right)$. Thus, the class

$$
\theta([a])=\left[f_{0}(b)\right] \in \operatorname{Coker}\left(\left(f_{0}\right)_{*}\right)
$$

is well defined. This gives a homomorphism

$$
\theta=\theta(K): \operatorname{Ker}\left(\left(f_{\infty}\right)_{*}\right) \longrightarrow \operatorname{Coker}\left(\left(f_{0}\right)_{*}\right) .
$$

Similarly, we define the map $\bar{\theta}=\bar{\theta}(K): \operatorname{Ker}\left(\left(\bar{f}_{\infty}\right)_{*}\right) \rightarrow \operatorname{Coker}\left(\left(\bar{f}_{0}\right)_{*}\right)$ from

$$
\bar{f}_{\infty}: C_{0} \longrightarrow \mathbb{C}_{1} \quad \text { and } \quad \bar{f}_{0}: C_{1} \longrightarrow C_{\infty}
$$

Proposition 4.6 The maps

$\theta(K): \operatorname{Ker}\left(\mathfrak{f}_{\infty}(K)\right) \longrightarrow \operatorname{Coker}\left(\mathfrak{f}_{0}(K)\right) \quad$ and $\quad \bar{\theta}(K): \operatorname{Ker}\left(\overline{\mathfrak{f}}_{\infty}(K)\right) \longrightarrow \operatorname{Coker}\left(\overline{\mathfrak{f}}_{0}(K)\right)$ 
are the inverses of the maps induced by $\mathfrak{f}_{1}(K), \overline{\mathfrak{f}}_{1}(K): \mathbb{H}_{\infty}(K) \rightarrow \mathbb{H}_{0}(K)$ which sit in the exact sequences:
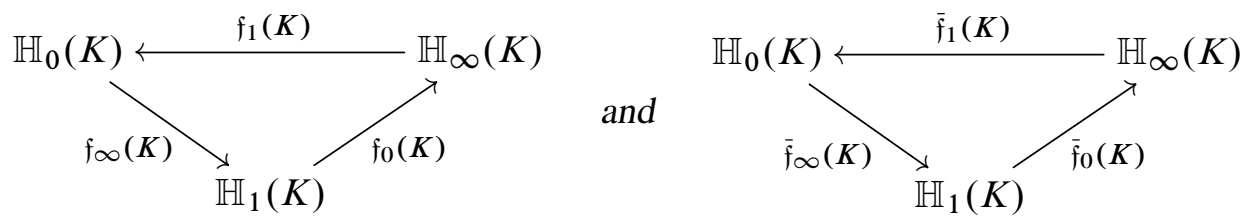

Proof For this purpose, let us assume that the Heegaard diagram

$$
\left(\Sigma, \boldsymbol{\alpha}, \hat{\boldsymbol{\beta}},\left\{\lambda_{0}, \lambda_{1}, \lambda_{\infty}\right\} ; u, v, w, \bar{u}, \bar{v}, \bar{w}\right)
$$

is constructed from a special Heegaard diagram as before. Let $\boldsymbol{\beta}$. for $\bullet \in\{0,1, \infty\}$ denote the set $\boldsymbol{\beta}_{\bullet}=\left\{\beta_{1}^{\bullet}, \ldots, \beta_{g-1}^{\bullet}, \lambda_{\bullet}\right\}$ constructed before. Let us furthermore assume that $\boldsymbol{\beta}_{1}^{\prime}$ is a set of $g$ simple closed curves, where the first $g-1$ of them are small Hamiltonian isotopes of the first $g-1$ curves in $\boldsymbol{\beta}_{1}$ (with two transverse intersection points with the corresponding simple closed curve in $\boldsymbol{\beta}_{1}$ ) while the last $\left(g^{\text {th }}\right)$ curve is denoted by $\lambda_{1}^{\prime}$. We assume that $\lambda_{1}^{\prime}$ is a Hamiltonian isotope of $\lambda_{1}$, which is very close to the juxtaposition of the curves $\lambda_{0}$ and $\lambda_{\infty}$.

Consider the two Heegaard quadruples

$$
H=\left(\Sigma, \boldsymbol{\alpha}, \boldsymbol{\beta}_{0}, \boldsymbol{\beta}_{1}, \boldsymbol{\beta}_{\infty} ; u, v, w\right) \quad \text { and } \quad H^{\prime}=\left(\Sigma, \boldsymbol{\alpha}, \boldsymbol{\beta}_{0}, \boldsymbol{\beta}_{1}^{\prime}, \boldsymbol{\beta}_{\infty} ; u, v, w\right) .
$$

Let us denote the triangle maps associated with the first Heegaard diagram by

$$
\begin{aligned}
\mathfrak{f}_{0}(H) & =\phi\left(H \backslash \boldsymbol{\beta}_{0}\right): C_{1}(K ; H) \longrightarrow C_{\infty}(K ; H), \\
\mathfrak{f}_{\infty}(H) & =\phi\left(H \backslash \boldsymbol{\beta}_{\infty}\right): C_{0}(K ; H) \longrightarrow C_{1}(K ; H),
\end{aligned}
$$

while the triangle maps associated with the Heegaard quadruple $H^{\prime}$ are denoted by

$$
\begin{aligned}
\mathfrak{f}_{0}\left(H^{\prime}\right) & =\phi\left(H^{\prime} \backslash \boldsymbol{\beta}_{0}\right): C_{1}\left(K ; H^{\prime}\right) \longrightarrow C_{\infty}\left(K ; H^{\prime}\right)=C_{\infty}(K ; H), \\
\mathfrak{f}_{\infty}\left(H^{\prime}\right) & =\phi\left(H^{\prime} \backslash \boldsymbol{\beta}_{\infty}\right): C_{0}\left(K ; H^{\prime}\right)=C_{0}(K ; H) \longrightarrow C_{1}\left(K ; H^{\prime}\right) .
\end{aligned}
$$

The holomorphic triangle map $\mathfrak{f}_{1}(H)=\mathfrak{f}_{1}\left(H^{\prime}\right): C_{\infty}(K ; H) \rightarrow C_{0}(K ; H)$ may be defined using the Heegaard triple $\left(\Sigma, \boldsymbol{\alpha}, \boldsymbol{\beta}_{\infty}, \boldsymbol{\beta}_{0} ; u, v, w\right)$. Count of the holomorphic rectangles in $H$ and $H^{\prime}$, respectively, that avoid the punctures $u, v$ and $w$ gives the homomorphisms

$$
\Phi_{1}: C_{0}(K ; H) \longrightarrow C_{\infty}(K ; H) \quad \text { and } \quad \Phi_{1}^{\prime}: C_{0}(K ; H) \longrightarrow C_{\infty}(K ; H)
$$

such that

$d_{\infty} \circ \Phi_{1}+\Phi_{1} \circ d_{0}=\mathfrak{f}_{0}(H) \circ \mathfrak{f}_{\infty}(H)$ and $d_{\infty} \circ \Phi_{1}^{\prime}+\Phi_{1}^{\prime} \circ d_{0}=\mathfrak{f}_{0}\left(H^{\prime}\right) \circ \mathfrak{f}_{\infty}\left(H^{\prime}\right)$. 
The interesting observation is that both $\Phi_{1}$ and $\Phi_{1}^{\prime}$ vanish when the Heegaard diagram is chosen as above. The reason for the first vanishing result is that there are no positive squares connecting the four intersection points

$$
\boldsymbol{x} \in \mathbb{T}_{\alpha} \cap \mathbb{T}_{\beta_{0}}, \quad \Theta_{0,1} \in \mathbb{T}_{\beta_{0}} \cap \mathbb{T}_{\beta_{1}}, \quad \Theta_{1, \infty} \in \mathbb{T}_{\beta_{1}} \cap \mathbb{T}_{\beta_{\infty}} \quad \text { and } \quad \boldsymbol{y} \in \mathbb{T}_{\beta_{\infty}} \cap \mathbb{T}_{\alpha} .
$$

In fact, $n_{\bar{u}}(\square)=n_{\bar{w}}(\square)=1$ for every square class $\square \in \pi_{2}^{+}\left(\boldsymbol{x}, \Theta_{0,1}, \Theta_{1, \infty}, \boldsymbol{y}\right)$. Thus, two opposite quarters around the intersection point $r_{1}$ have zero coefficient, while one other quadrant has coefficient 1 . Since $r_{1}$ is not among the intersection points in any of $\boldsymbol{x}, \boldsymbol{y}, \Theta_{0,1}$ and $\Theta_{1, \infty}$, the coefficient of the last quadrant around $r_{1}$ is -1 and the contribution of $\square$ is thus trivial. A similar argument implies that $\Phi_{1}^{\prime}$ is zero.

For $\bullet \in\{0,1, \infty\}$, the Heegaard triple $H_{\bullet}=\left(\Sigma, \boldsymbol{\alpha}, \boldsymbol{\beta}_{\bullet}^{\prime}, \boldsymbol{\beta}_{\bullet} ; u, v, w\right)$ gives

$$
l_{\bullet}=l_{(}\left(H_{\bullet}\right): C_{\bullet}\left(K ; H^{\prime}\right) \longrightarrow C_{\bullet}(K ; H) .
$$

The homomorphisms $l_{0}$ and $l_{\infty}$ are the identity maps of $C_{0}(K ; H)$ and $C_{\infty}(K ; H)$, respectively. The Heegaard quadruple

$$
\left(\Sigma, \boldsymbol{\alpha}, \boldsymbol{\beta}_{0}, \boldsymbol{\beta}_{1}^{\prime}, \boldsymbol{\beta}_{1} ; u, v, w\right)
$$

determines a holomorphic square map

$$
\Psi_{\infty}: C_{0}(K ; H) \longrightarrow C_{1}(K ; H) .
$$

Considering different possible degenerations of a holomorphic square of Maslov index zero, one finds the relation

$$
d_{1} \circ \Psi_{\infty}+\Psi_{\infty} \circ d_{0}=l_{1} \circ \mathfrak{f}_{\infty}\left(H^{\prime}\right)+\mathfrak{f}_{\infty}(H) .
$$

Finally, one may consider the Heegaard 5-tuple

$$
\left(\Sigma, \boldsymbol{\alpha}, \boldsymbol{\beta}_{0}, \boldsymbol{\beta}_{1}^{\prime}, \boldsymbol{\beta}_{1}, \boldsymbol{\beta}_{\infty} ; u, v, w\right),
$$

which may be used to construct a pentagon map $Q: C_{0}(K ; H) \rightarrow C_{\infty}(K ; H)$. Considering all possible degenerations of a holomorphic pentagon of Maslov index -1 , one obtains the relation

$$
d_{\infty} \circ Q+Q \circ d_{0}=\Psi_{0} \circ \mathfrak{f}_{\infty}\left(H^{\prime}\right)+\mathfrak{f}_{0}(H) \circ \Psi_{\infty},
$$

where $\Psi_{0}: C_{1}\left(K ; H^{\prime}\right) \rightarrow C_{\infty}(K ; H)$ is the holomorphic square map associated with $\left(\Sigma, \boldsymbol{\alpha}, \boldsymbol{\beta}_{1}^{\prime}, \boldsymbol{\beta}_{1}, \boldsymbol{\beta}_{\infty} ; u, v, w\right)$. The reason for the above equality is that the contributing holomorphic squares in the Heegaard quadruple $\left(\Sigma, \boldsymbol{\beta}_{0}, \boldsymbol{\beta}_{1}^{\prime}, \boldsymbol{\beta}_{1}, \boldsymbol{\beta}_{\infty} ; u, v, w\right)$ come in cancelling pairs, while there is a single contributing holomorphic triangle corresponding to each of the Heegaard triples

$$
\left(\Sigma, \boldsymbol{\beta}_{1}^{\prime}, \boldsymbol{\beta}_{1}, \boldsymbol{\beta}_{\infty} ; u, v, w\right) \text { and }\left(\Sigma, \boldsymbol{\beta}_{0}, \boldsymbol{\beta}_{1}^{\prime}, \boldsymbol{\beta}_{1} ; u, v, w\right) .
$$



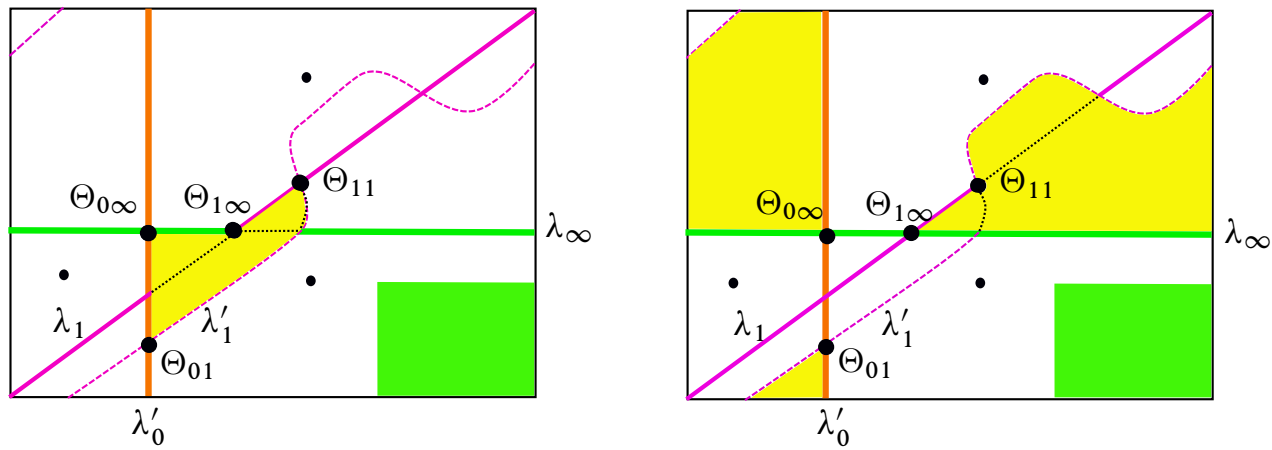

Figure 8: The domains for a cancelling pair of contributing squares connecting $\Theta_{01}, \Theta_{1,1}, \Theta_{1, \infty}$ and $\Theta_{0, \infty}$

Figure 8 illustrates the domain for a cancelling pair of contributing squares. Moreover, the maps $\Phi_{1}$ and $\Phi_{1}^{\prime}$, which may potentially contribute, are trivial.

Our choice of $\lambda_{1}^{\prime}$ and the fact that the Heegaard diagram is nice imply that we have a short exact sequence

$$
0 \longrightarrow C_{0}(K ; H) \stackrel{\mathfrak{f}_{\infty}\left(H^{\prime}\right)}{\longrightarrow} C_{1}\left(K ; H^{\prime}\right) \stackrel{\mathfrak{f}_{0}\left(H^{\prime}\right)}{\longrightarrow} C_{\infty}(K ; H) \longrightarrow 0 .
$$

Correspondingly, an isomorphism $\theta^{\prime}: \operatorname{Ker}\left(\mathfrak{f}_{\infty}(K)\right) \rightarrow \operatorname{Coker}\left(\mathfrak{f}_{0}(K)\right)$ may be constructed. Choose some closed element $a \in C_{0}(K ; H)$ and let $\mathfrak{f}_{\infty}\left(H^{\prime}\right)(a)=d_{1}^{\prime}\left(b^{\prime}\right)$ for some $b^{\prime} \in C_{1}\left(K ; H^{\prime}\right)$. By $(15)$,

$$
\mathfrak{f}_{\infty}(H)(a)=\left(l_{1} \circ \mathfrak{f}_{\infty}\left(H^{\prime}\right)+d_{1} \circ \Psi_{\infty}\right)(a)=d_{1}\left(l_{1}\left(b^{\prime}\right)+\Psi_{\infty}(a)\right)=: d_{1}(b) .
$$

Using (15) and (16) we compute

$$
\begin{aligned}
\mathfrak{f}_{0}(H)(b) & =\mathfrak{f}_{0}(H)\left(l_{1}\left(b^{\prime}\right)+\Psi_{\infty}(a)\right) \\
& =\mathfrak{f}_{0}\left(H^{\prime}\right)\left(b^{\prime}\right)+\left(d_{\infty} \circ \Psi_{0}+\Psi_{0} \circ d_{1}^{\prime}\right)\left(b^{\prime}\right)+\left(\mathfrak{f}_{0}(H) \circ \Psi_{\infty}\right)(a) \\
& =\mathfrak{f}_{0}\left(H^{\prime}\right)\left(b^{\prime}\right)+d_{\infty} \circ \Psi_{0}\left(b^{\prime}\right)+\left(\Psi_{0} \circ \mathfrak{f}_{\infty}\left(H^{\prime}\right)+\mathfrak{f}_{0}(H) \circ \Psi_{\infty}\right)(a) \\
& =\mathfrak{f}_{0}\left(H^{\prime}\right)\left(b^{\prime}\right)+d_{\infty}\left(Q(a)+\Psi_{0}\left(b^{\prime}\right)\right)
\end{aligned}
$$

This means that the maps $\theta(K)$ and $\theta^{\prime}$, as maps from $\operatorname{Ker}\left(\mathfrak{f}_{\infty}(K)\right)$ to $\operatorname{Coker}\left(\mathfrak{f}_{0}(K)\right)$, are the same. However, the map $\theta^{\prime}$ is the inverse of the connecting homomorphism $\delta: \operatorname{Coker}\left(\mathfrak{f}_{0}(K)\right) \rightarrow \operatorname{Ker}\left(\mathfrak{f}_{\infty}(K)\right)$ resulting from the short exact sequence

$$
0 \longrightarrow C_{0}(K ; H) \stackrel{\mathfrak{f}_{\infty}\left(H^{\prime}\right)}{\longrightarrow} C_{1}\left(K ; H^{\prime}\right) \stackrel{\mathfrak{f}_{0}\left(H^{\prime}\right)}{\longrightarrow} C_{\infty}(K ; H) \longrightarrow 0 .
$$


The above observations imply the claim for $\theta(K)$. The proof for the map $\bar{\theta}(K)$ is similarly reduced to showing the triviality of the holomorphic square map corresponding to the Heegaard quadruple $\left(\Sigma, \boldsymbol{\alpha}, \boldsymbol{\beta}_{0}, \boldsymbol{\beta}_{1}, \boldsymbol{\beta}_{\infty} ; \bar{u}, \bar{v}, \bar{w}\right)$.

The domain of every contributing holomorphic square corresponding to the aforementioned punctured Heegaard diagram has coefficient zero at $\bar{u}, \bar{v}, \bar{w}$ and $w$, and coefficient 1 at $u$ and $v$. This implies that two opposite quadrants around $r_{3}$ have coefficient zero, while a third quadrant has coefficient 1 . Since $r_{3}$ cannot be among the intersection points on the vertices of the square, the fourth quadrant around $r_{3}$ has coefficient -1 . This contradiction gives the triviality of the holomorphic square map corresponding to $\left(\Sigma, \boldsymbol{\alpha}, \boldsymbol{\beta}_{0}, \boldsymbol{\beta}_{1}, \boldsymbol{\beta}_{\infty} ; \bar{u}, \bar{v}, \bar{w}\right)$ and completes the proof.

\section{Gluing the knot complements}

\subsection{Extracting a chain complex for splicing}

Given two Heegaard diagrams for the complements of the knots $K_{1}$ and $K_{2}$, one may construct a Heegaard diagram for $Y\left(K_{1}, K_{2}\right)$ as follows, similar to the construction of Eftekhary [2]. Let

$$
H_{i}=\left(\Sigma_{i}, \boldsymbol{\alpha}^{i}, \hat{\boldsymbol{\beta}}^{i} \cup\left\{\mu_{i}, \lambda_{i}\right\}\right)
$$

denote the Heegaard diagram for $K_{i}$ with Heegaard surface $\Sigma_{i}$, and with $\mu_{i}$ the meridian for $K_{i}$ and $\lambda_{i}$ a zero-framed longitude for it which cuts $\mu_{i}$ in a single point $O_{i}$. Then the Heegaard diagram for the three-manifold $Y=Y\left(K_{1}, K_{2}\right)$ obtained by splicing the complement of $K_{1} \subset Y_{1}$ and the complement of $K_{2} \subset Y_{2}$ is constructed as follows. Attach a 1-handle to $\Sigma_{1} \cup \Sigma_{2}$, with attaching circles placed at the intersections $O_{1}$ and $\mathrm{O}_{2}$. Use four parallel segments on this 1-handle to connect the four intersections of $\mu_{1} \cup \lambda_{1}$ with one of the attaching circles to the four intersections of $\mu_{2} \cup \lambda_{2}$ with the other attaching circle, so that intersection points on $\mu_{1}$ are joined to the intersection points on $\lambda_{2}$. The union of the remaining parts from $\mu_{1}$ and $\lambda_{2}$ with two of the four parallel line segments gives a simple closed curve on $\Sigma$, which will be denoted by $\mu_{1} \# \lambda_{2}$. The simple closed curve $\lambda_{1} \# \mu_{2}$ is constructed in a similar way. Let

$$
\boldsymbol{\alpha}=\boldsymbol{\alpha}^{1} \cup \boldsymbol{\alpha}^{2} \text { and } \boldsymbol{\beta}=\widehat{\boldsymbol{\beta}}^{1} \cup \widehat{\boldsymbol{\beta}}^{2} \cup\left\{\mu_{1} \# \lambda_{2}, \lambda_{1} \# \mu_{2}\right\} .
$$

The resulting Heegaard diagram $H=(\Sigma, \boldsymbol{\alpha}, \boldsymbol{\beta})$ is a Heegaard diagram for the threemanifold obtained by splicing the two knot complements.

If the initial Heegaard diagrams $H_{i}$ are special (see Definition 4.2) one may assume that the Heegaard diagram $H$ will have one bad region and the rest of the regions are either bigons or rectangles. Thus, the combinatorial algorithm of Sarkar and Wang [16] 


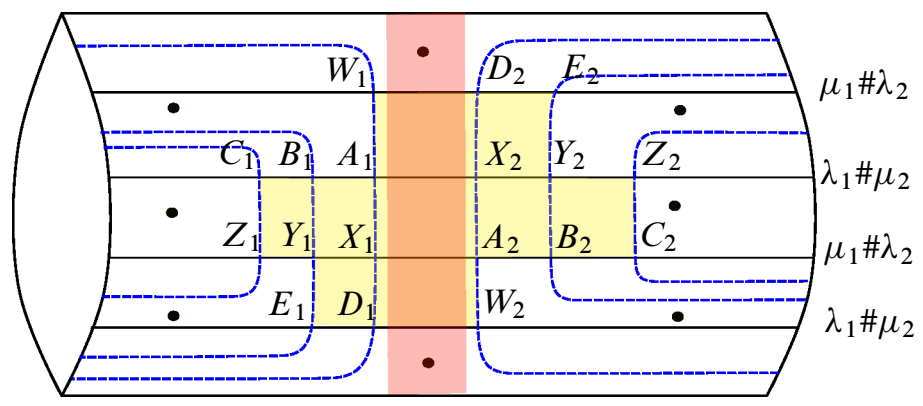

Figure 9: The cylinder illustrates a neighbourhood of the 1-handle used for attaching the two Heegaard diagrams. The union of the domains of the disks intersecting the 1-handle and contributing to the differential is shaded yellow.

may be used to compute its (hat) Heegaard Floer homology with $\mathbb{F}$ coefficients. Let $z$ denote a marked point which is placed in the aforementioned bad region. The marked point $z$ corresponds to the marked points $z_{i} \in \Sigma_{i}, i=1,2$. We may also choose a second marked point $z_{i}^{\prime}$ for the Heegaard diagram $H_{i}$ which is placed next to $O_{i}$ and in the quadrant opposite to the quadrant containing $z_{i}$.

Define the chain complexes $M^{i}$ and $L^{i}$ associated with $K_{i} \subset Y_{i}$ using the Heegaard diagrams

$$
\left(\Sigma_{i}, \boldsymbol{\alpha}^{i}, \hat{\boldsymbol{\beta}}^{i} \cup\left\{\mu_{i}\right\} ; z_{i}, z_{i}^{\prime}\right) \text { and }\left(\Sigma_{i}, \boldsymbol{\alpha}^{i}, \widehat{\boldsymbol{\beta}}^{i} \cup\left\{\lambda_{i}\right\} ; z_{i}, z_{i}^{\prime}\right),
$$

respectively. Note that the generators of the complex $C$ associated with the Heegaard diagram $H$ are in correspondence, either with the generators of $M=M^{1} \otimes M^{2}$ or the generators of $L=L^{1} \otimes L^{2}$, ie the $\mathbb{F}$-module $C$ may be identified with $M \oplus L$. Denote the differential of $M$ by $d_{M}$ and the differential of $L$ by $d_{L}$. The domain of every disk which contributes to the differential of $C$ is then a rectangle or a bigon in the diagram. Such a disk may either stay in one of the $\Sigma_{i}$ or intersect both $\Sigma_{1}$ and $\Sigma_{2}$. The disks that stay in one of the $\Sigma_{i}$ correspond to the differentials $d_{M}$ and $d_{L}$ of the complexes $M$ and $L$. Only a few rectangles can intersect both $\Sigma_{i}$ and miss the marked point $z$ (see Figure 9), while no bigons can intersect both $\Sigma_{1}$ and $\Sigma_{2}$. Because of the way the bad region (the region containing the marked point) enters the neighbourhood of the 1-handle, the rectangles which intersect both $\Sigma_{1}$ and $\Sigma_{2}$ stay in the neighbourhood of the 1-handle. The contribution of such rectangles may be described after introducing some extra notation.

The assumption on the Heegaard diagrams $H_{1}$ and $H_{2}$ from Lemma 4.1 implies that the local picture around $O_{i}$ looks like the genus- 4 surface illustrated on the top of Figure 3. Denote the intersection points on $H_{i}$ which correspond to $A, B, C, D, E$, $X, Y, Z$ and $W$ by $A_{i}, B_{i}, C_{i}, D_{i}, E_{i}, X_{i}, Y_{i}, Z_{i}$ and $W_{i}$, respectively. 
The generators of $M^{i} \oplus L^{i}$ are the tuples $\boldsymbol{x}=\left(x_{1}, \ldots, x_{g_{i}}\right)$ such that, for a permutation $\sigma:\left\{1, \ldots, g_{i}\right\} \rightarrow\left\{1, \ldots, g_{i}\right\}$, we have $x_{j} \in \alpha_{\sigma(j)} \cap \beta_{j}$ for $j=1, \ldots, g_{i}-1$ and $x_{g_{i}} \in \alpha_{\sigma\left(g_{i}\right)} \cap\left(\mu_{i} \cup \lambda_{i}\right)$. The complex $M^{i}$ is generated by those $\boldsymbol{x}$ such that $x_{g_{i}} \in \mu_{i}$, and the complex $L^{i}$ is generated by the $g_{i}$-tuples $\boldsymbol{x}=\left(x_{1}, \ldots, x_{g_{i}}\right)$ with $x_{g_{i}} \in \lambda_{i}$. The homology of the complex $M^{i}$ is the knot Floer homology $\widehat{\operatorname{HFK}}\left(K_{i}\right)$ and the homology of the complex $L^{i}$ is the longitude Floer homology $\operatorname{HFL}\left(K_{i}\right)$. The homomorphisms $\Phi^{i}: M^{i} \rightarrow L^{i}$ over $\boldsymbol{x}=\left(x_{1}, \ldots, x_{g_{i}}\right) \in M^{i}$ are defined by

$$
\Phi^{i}(\boldsymbol{x})= \begin{cases}\left(x_{1}, \ldots, x_{g_{i}-1}, X_{i}\right) & \text { if } x_{g_{i}}=A_{i}, \\ \left(x_{1}, \ldots, x_{g_{i}-1}, Y_{i}\right) & \text { if } x_{g_{i}}=B_{i}, \\ \left(x_{1}, \ldots, x_{g_{i}-1}, Z_{i}\right) & \text { if } x_{g_{i}}=C_{i}, \\ 0 & \text { otherwise }\end{cases}
$$

The corresponding contributing triangles are $\left[A_{i} O_{i} X_{i}\right],\left[B_{i} O_{i} Y_{i}\right]$ and $\left[C_{i} O_{i} Z_{i}\right]$. The map $\Phi$ thus corresponds to the changes $x_{g_{i}} \rightarrow y_{g_{i}}$ which are one of the following: $A_{i} \rightarrow X_{i}, B_{i} \rightarrow Y_{i}$ or $C_{i} \rightarrow Z_{i}$. Similarly, the homomorphisms $\Psi_{1}^{i}: L^{i} \rightarrow M^{i}$ correspond to the triangles $\left[W_{i} O_{i} A_{i}\right]$ and, over $\boldsymbol{x}=\left(x_{1}, \ldots, x_{g_{i}}\right) \in L^{i}$, are defined by

$$
\Psi_{1}^{i}(\boldsymbol{x})= \begin{cases}\left(x_{1}, \ldots, x_{g_{i}-1}, A_{i}\right) & \text { if } x_{g_{i}}=W_{i} \\ 0 & \text { otherwise }\end{cases}
$$

Define the maps $\Psi_{2}^{i}, \Psi_{3}^{i}: L^{i} \rightarrow M^{i}$, where $\Psi_{2}^{i}$ corresponds to the changes $X_{i} \rightarrow D_{i}$ and $Y_{i} \rightarrow E_{i}$, and $\Psi_{3}^{i}$ corresponds to $W_{i} \rightarrow D_{i}$. Thus the triangles contributing to $\Psi_{2}^{i}$ are $\left[X_{i} O_{i} D_{i}\right]$ and $\left[Y_{i} O_{i} E_{i}\right]$, while the only triangle contributing to $\Psi_{3}^{i}$ is

$$
\left[W_{i} O_{i} D_{i}\right]=\left[W_{i} O_{i} A_{i}\right] \cup\left[A_{i} O_{i} X_{i}\right] \cup\left[X_{i} O_{i} D_{i}\right] .
$$

The contribution of the rectangles which intersect both $\Sigma_{1}$ and $\Sigma_{2}$ to the differential of the complex $C=M \oplus L$ may thus be described by the maps

$$
\left.\begin{array}{rl} 
& \Phi=\Phi^{1} \otimes \Phi^{2}: L^{1} \otimes L^{2} \longrightarrow M^{1} \otimes M^{2}, \\
\Psi_{1} & =\Psi_{1}^{1} \otimes \Psi_{2}^{2} \\
\Psi_{2} & =\Psi_{2}^{1} \otimes \Psi_{1}^{2} \\
\Psi_{3} & =\Psi_{3}^{1} \otimes \Psi_{3}^{2}
\end{array}\right\}: M^{1} \otimes M^{2} \longrightarrow L^{1} \otimes L^{2} .
$$

In other words, the differential of the complex $C=M \oplus L$ is the homomorphism

$$
d=d_{C}=\left(\begin{array}{cc}
d_{M} & \Phi \\
\sum_{i=1}^{3} \Psi_{i} & d_{L}
\end{array}\right) .
$$

Proposition 5.1 The complexes $M^{i}$ and $L^{i}$ are identified with the mapping cones of $\bar{f}_{\infty}^{i}=\bar{f}_{\infty}\left(K_{i}\right)$ and $f_{0}^{i}=f_{0}\left(K_{i}\right)$, respectively. More precisely, the $\mathbb{F}$-module $M^{i}$ is isomorphic to the direct sum of $C_{1}\left(K_{i}\right)$ and $C_{0}\left(K_{i}\right)$, while $L^{i}$ is isomorphic to the 
direct sum of $C_{\infty}\left(K_{i}\right)$ and $C_{1}\left(K_{i}\right)$. Moreover, the differentials $d_{M^{i}}$ and $d_{L^{i}}$ of $M^{i}$ and $L^{i}$ are identified as

$$
\begin{array}{ll}
d_{L_{i}}\left(c_{1}, c_{\infty}\right)=\left(d_{1}^{i}\left(c_{1}\right), d_{\infty}^{i}\left(c_{\infty}\right)+f_{0}^{i}\left(c_{1}\right)\right) & \text { for all }\left(c_{1}, c_{\infty}\right) \in C_{1}\left(K_{i}\right) \oplus C_{\infty}\left(K_{i}\right), \\
d_{M_{i}}\left(c_{0}, c_{1}\right)=\left(d_{0}^{i}\left(c_{0}\right), d_{1}^{i}\left(c_{1}\right)+\bar{f}_{\infty}^{i}\left(c_{0}\right)\right) & \text { for all }\left(c_{0}, c_{1}\right) \in C_{0}\left(K_{i}\right) \oplus C_{1}\left(K_{i}\right) .
\end{array}
$$

Proof We sketch the proof of the claim for $L^{i}$. The corresponding claim for $M^{i}$ is proved in a completely similar way. Consider the labelling of the intersection points of the $\alpha$-curves with the curves $\lambda_{\infty}\left(K_{i}\right), \lambda_{1}\left(K_{i}\right)$ and $\lambda\left(K_{i}\right)$ as in Figure 5 . The intersection points with the $\alpha$-curves on $\lambda_{1}\left(K_{i}\right)$ are $r_{1}, r_{2}$ and $r_{3}$. The intersection points with the $\alpha$-curves on $\lambda_{\infty}\left(K_{i}\right)$ are $s_{1}, s_{2}, \ldots, s_{n}, q_{3}, q_{4}, \ldots, q_{n}$ and the intersection points with the $\alpha$-curves on $\lambda\left(K_{i}\right)$ are $S_{1}, S_{2}, \ldots, S_{n}, Q_{3}, Q_{4}, \ldots, Q_{n}$ and $R_{1}, R_{2}, R_{3}$. Define the $\mathbb{F}$-module isomorphism

$$
I_{i}: C_{1}\left(K_{i}\right) \oplus C_{\infty}\left(K_{i}\right) \longrightarrow L^{i}, \quad I_{i}\left(x=\left(x_{1}, \ldots, x_{g_{i}}\right)\right):=\left(x_{1}, \ldots, x_{g_{i}-1}, I_{i}\left(x_{g_{i}}\right)\right),
$$

where $I_{i}$ changes the letter in the labelling of an intersection point to a capital letter (so $I_{i}\left(r_{j}\right)=R_{j}, I_{i}\left(s_{j}\right)=S_{j}$ and $\left.I_{i}\left(q_{j}\right)=Q_{j}\right)$. Straightforward combinatorics may be used to verify $d_{L^{i}}\left(I_{i}(\boldsymbol{x})\right)=I_{i}\left(d_{\infty}^{i}(\boldsymbol{x})\right)$ for every generator $\boldsymbol{x}$ of $C_{\infty}\left(K_{i}\right)$ and $d_{L^{i}}\left(I_{i}(\boldsymbol{x})\right)=I_{i}\left(d_{1}^{i}(\boldsymbol{x})\right)+I_{i}\left(f_{0}^{i}(\boldsymbol{x})\right)$ for every generator $\boldsymbol{x}$ of $C_{1}\left(K_{i}\right)$.

Under the identification of $M^{i}$ with the mapping cone of $\bar{f}_{\infty}^{i}$ and the identification of $L^{i}$ with the mapping cone of $f_{0}^{i}$, the map $\Phi$ has a simple description: it is the map that takes $C_{1}\left(K_{i}\right)$ in the mapping cone of $f_{0}: C_{1}\left(K_{i}\right) \rightarrow C_{\infty}\left(K_{i}\right)$ to the complex $C_{1}\left(K_{i}\right)$ in the mapping cone of $\bar{f}_{\infty}^{i}: C_{0}\left(K_{i}\right) \rightarrow C_{1}\left(K_{i}\right)$ via the identity map of $C_{1}\left(K_{i}\right)$. Furthermore, the map $f_{\infty}^{i}$ from $C_{0}\left(K_{i}\right)$ in $M^{i}$ to $C_{1}\left(K_{i}\right)$ in $L^{i}$ is identified with the triangle map $\Psi_{1}^{i}$. The induced map $\bar{f}_{0}^{i}$ from the copy of $C_{1}\left(K_{i}\right)$ in $M$ to the copy of $C_{\infty}\left(K_{i}\right)$ in $L^{i}$ is the triangle map $\Psi_{2}^{i}$. The map $\Psi_{3}^{i}$ is obtained from the composition map $\bar{f}_{0}^{i} \circ f_{\infty}^{i}: C_{0}\left(K_{i}\right) \rightarrow C_{\infty}\left(K_{i}\right)$. Set $C_{\bullet}^{i}=C_{\bullet}\left(K_{i}\right)$. If we replace the mapping cone of $f_{0}^{i}: C_{1}^{i} \rightarrow C_{\infty}^{i}$ for $L^{i}$, replace the mapping cone $\bar{f}_{\infty}^{i}: C_{0}^{i} \rightarrow C_{1}^{i}$ for $M^{i}$, and also replace $\Phi^{i}$ and $\Psi_{j}^{i}$ with the appropriate descriptions in terms of $\bar{f}_{0}^{i}$ and $f_{\infty}^{i}$, we obtain an alternative description of the complex $C$.

The cube 四 $=$ 四 $\left(f_{\bullet}^{i}, \bar{f}_{\bullet}^{i} \mid \bullet=0, \infty, i=1,2\right)$ associated with the knots $K_{1}$ and $K_{2}$, the corresponding complexes $C_{\bullet}^{i}, i=1,2, \bullet \in\{0,1, \infty\}$, and the maps $f_{0}^{i}, \bar{f}_{0}^{i}: C_{1}^{i} \rightarrow C_{\infty}^{i}$ and $f_{\infty}^{i}, \bar{f}_{\infty}^{i}: C_{0}^{i} \rightarrow C_{1}^{i}$ is the chain complex (四, $d_{\text {四 }}$ ) associated with the graph of 
complexes represented by the following cube:

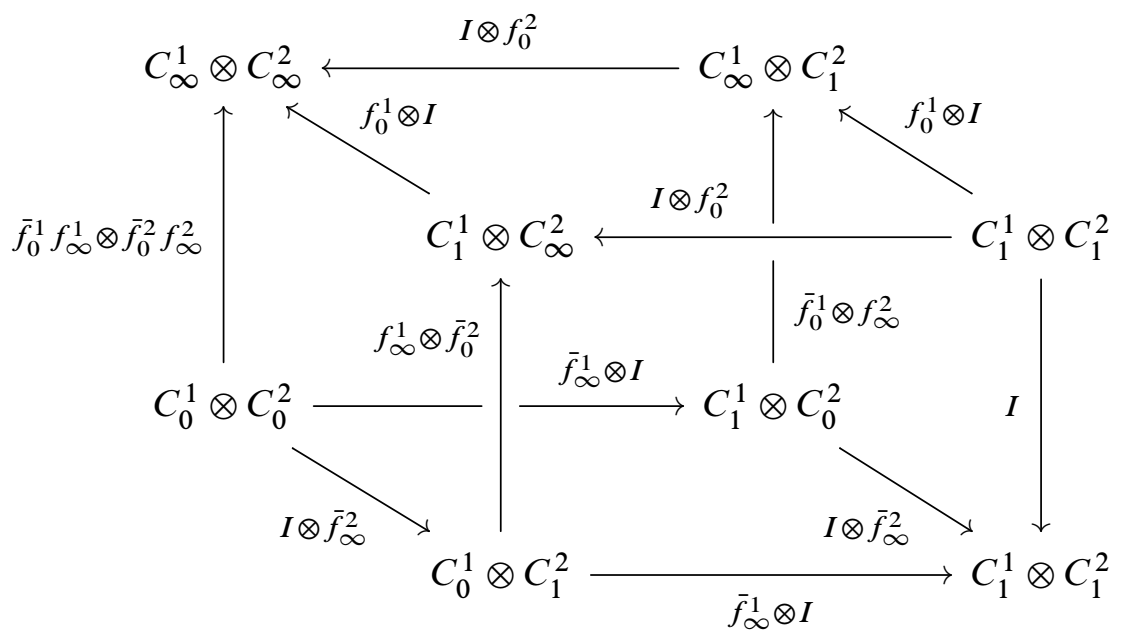

Proposition 5.2 With the above notation fixed, the complex $(C, d)$ is identified, as a chain complex, with the cube

$$
\text { (四 } \left.=\text { 四 }\left(f_{\bullet}^{i}, \bar{f}_{\bullet}^{i} \mid \cdot=0, \infty, i=1,2\right), d_{\text {四 }}\right) .
$$

\subsection{The linear algebra of the cubes}

Let $\mathbb{H}_{\bullet}^{i}$ denote the homology of the chain complex $\left(C_{\bullet}^{i}, d_{\bullet}^{i}\right)$ for $i=1,2, \bullet \in\{0,1, \infty\}$. Set $\mathbb{H}_{\bullet, \star}=\mathbb{H}_{\bullet}^{1} \otimes \mathbb{H}_{\star}^{2}$ for $\bullet, \star \in\{0,1, \infty\}$. Abusing the notation, the map induced on homology by $f_{\bullet}^{i}$ will also be denoted $\mathfrak{f}_{\bullet}^{i}$ and the map induced on homology by $\bar{f}_{\bullet}^{i}$ will be denoted $\overline{\mathrm{f}}_{\bullet}^{i}$.

Following the discussion of Section 5.3, we may choose appropriate decompositions $C_{\bullet}^{i}=A_{\bullet}^{i} \oplus \mathbb{H}_{\bullet}^{i} \oplus A_{\bullet}^{i}$ such that the differential $d_{\bullet}^{i}$ takes the form

$$
d_{\bullet}^{i}=\left(\begin{array}{lll}
0 & 0 & I \\
0 & 0 & 0 \\
0 & 0 & 0
\end{array}\right) .
$$

Correspondingly, we find the matrices $G\left(f_{\bullet}^{i}\right)=\left(f_{\bullet}^{i}\right)_{*}$ and $G\left(\bar{f}_{\bullet}^{i}\right)=\left(\bar{f}_{\bullet}^{i}\right)_{*}$, which will be denoted by $\mathfrak{f}_{\bullet}^{i}$ and $\overline{\mathfrak{f}}_{\bullet}^{i}$, as well as the matrices

$$
M\left(f_{\bullet}^{i}\right), \quad M\left(\bar{f}_{\bullet}^{i}\right), \quad P\left(f_{\bullet}^{i}\right), \quad P\left(\bar{f}_{\bullet}^{i}\right), \quad Q\left(f_{\bullet}^{i}\right), \quad Q\left(\bar{f}_{\bullet}^{i}\right), \quad N\left(f_{\bullet}^{i}\right) \text { and } \quad N\left(\bar{f}_{\bullet}^{i}\right) .
$$

The maps $Q\left(\mathfrak{f}_{0}^{i}\right) P\left(\mathfrak{f}_{\infty}^{i}\right)$ and $Q\left(\overline{\mathfrak{f}}_{0}^{i}\right) P\left(\overline{\mathfrak{f}}_{\infty}^{i}\right)$ from $\mathbb{H}_{0}^{i}$ to $\mathbb{H}_{\infty}^{i}$ extend the homomorphisms $\theta^{i}: \operatorname{Ker}\left(\mathfrak{f}_{\infty}^{i}\right) \longrightarrow \operatorname{Coker}\left(\mathfrak{f}_{0}^{i}\right)$ and $\bar{\theta}^{i}: \operatorname{Ker}\left(\overline{\mathfrak{f}}_{\infty}^{i}\right) \longrightarrow \operatorname{Coker}\left(\overline{\mathfrak{f}}_{0}^{i}\right)$, 
associated with the knot $K_{i} \subset Y_{i}$. These extensions are still denoted by $\theta^{i}$ and $\bar{\theta}^{i}$, respectively.

Lemma 2.4 implies that the homology of $\left(\right.$ 四,$\left.d_{\text {四 }}\right)$ is isomorphic to the homology of the chain complex $\left(\mathbb{H}, d_{\mathbb{H}}\right)$ associated with the graph of chain complexes determined by the cube of Figure 2 .

Proposition 5.3 Let $\left(\mathbb{H}, d_{\mathbb{H}}\right)$ denote the complex obtained from the cube (四, $d_{\text {四 }}$ ) by applying Lemma 2.4. Then $\left(\mathbb{H}, d_{\mathbb{H}}\right)$ is identified with the complex shown in Figure 2 provided that the maps $\theta^{i}=\theta\left(K_{i}\right)$ and $\bar{\theta}^{i}=\bar{\theta}\left(K_{i}\right)$ are given as above.

Proof If Lemma 2.4 is applied, we obtain the same oriented graph (ie the same new edges) and the same complexes on the vertices. The directed edge from $\mathbb{H}_{0,0}$ to $\mathbb{H}_{\infty, \infty}$ is labelled by the map

$\overline{\mathfrak{f}}_{0}^{1} \mathfrak{f}_{\infty}^{1} \otimes \overline{\mathfrak{f}}_{0}^{2} \mathfrak{f}_{\infty}^{2}+Q\left(f_{0}^{1} \otimes I\right) N\left(f_{\infty}^{1} \otimes \bar{f}_{0}^{2}\right) P\left(I \otimes \bar{f}_{\infty}^{2}\right)+Q\left(I \otimes f_{0}^{2}\right) N\left(\bar{f}_{0}^{1} \otimes f_{\infty}^{2}\right) P\left(\bar{f}_{\infty}^{1} \otimes I\right)$,

which is, by Lemma 2.6, equal to

$$
\overline{\mathfrak{f}}_{0}^{1} \mathfrak{f}_{\infty}^{1} \otimes \overline{\mathfrak{f}}_{0}^{2} \mathfrak{f}_{\infty}^{2}+\theta^{1} \otimes \bar{\theta}^{2}+\bar{\theta}^{1} \otimes \theta^{2}
$$

The map corresponding to the dashed edge from $\mathbb{H}_{0,0}$ to $\mathbb{H}_{1, \infty}$ is, by Lemma 2.5,

$$
Q\left(f_{\infty}^{1} \otimes \bar{f}_{0}^{2}\right) P\left(I \otimes \bar{f}_{\infty}^{2}\right)=\mathfrak{f}_{\infty}^{1} \otimes\left(Q\left(\bar{f}_{0}^{2}\right) P\left(\bar{f}_{\infty}^{2}\right)\right)=\mathfrak{f}_{\infty}^{1} \otimes \bar{\theta}^{2} .
$$

The maps corresponding to the rest of dashed directed edges may be computed in a completely similar way. This completes the proof of Proposition 5.3.

Remark 5.4 (1) Note that $Y\left(K_{1}, K_{2}\right)=Y\left(-K_{1},-K_{2}\right)$. One may assume that $\mathfrak{f}_{\bullet}(-K)=\overline{\mathfrak{f}}_{\bullet}(K)$ and $\overline{\mathfrak{f}}_{\bullet}(-K)=\mathfrak{f}_{\bullet}(K)$, implying that $\widehat{\operatorname{HF}}\left(Y\left(-K_{1},-K_{2}\right)\right)$ is isomorphic to the homology of the complex determined by the oriented graph in Figure 2, where all barred maps change to the corresponding unbarred maps and all unbarred maps change to the corresponding barred maps.

(2) Proposition 5.3 is still weaker than Theorem 1.1, since the extensions of $\theta^{i}$ and $\bar{\theta}^{i}$ to maps from $\mathbb{H}_{0}^{i}$ to $\mathbb{H}_{\infty}^{i}$ are not arbitrary yet. In fact, without freedom in choosing these two extensions (which will be proved by the end of the current section) Theorem 1.1 stays bound to the information from the corresponding nice Heegaard diagram and has much less significance. 


\subsection{Simplifications of the splicing formula}

We now apply Lemma 2.3 to the splicing formula of Proposition 5.3 and make some cancellations. The first cancellation comes from setting $C=\mathbb{H}, A=\mathbb{H}_{1,1}$ and

$$
B=\left(\mathbb{H}_{\infty, \infty} \oplus \mathbb{H}_{1, \infty} \oplus \mathbb{H}_{\infty, 1}\right) \oplus\left(\mathbb{H}_{0,1} \oplus \mathbb{H}_{1,0} \oplus \mathbb{H}_{0,0}\right)=\mathbb{E}_{1} \oplus \mathbb{E}_{2}
$$

We thus have $\widehat{\operatorname{HF}}(Y)=H_{*}\left(B, d_{B}\right)$, where

$$
d_{B}=\left(\begin{array}{cccccc}
0 & \mathfrak{f}_{0}^{1} \otimes I & I \otimes \mathfrak{f}_{0}^{2} & \theta^{1} \otimes \overline{\mathfrak{f}}_{0}^{2} & \overline{\mathfrak{f}}_{0}^{1} \otimes \theta^{2} & \Gamma \\
0 & 0 & 0 & \Phi & I \otimes\left(\mathfrak{f}_{0}^{2} \circ \overline{\mathfrak{f}}_{\infty}^{2}\right) & \mathfrak{f}_{\infty}^{1} \otimes \bar{\theta}^{2} \\
0 & 0 & 0 & \left(\mathfrak{f}_{0}^{1} \circ \overline{\mathfrak{f}}_{\infty}^{1}\right) \otimes I & \Psi & \bar{\theta}^{1} \otimes \mathfrak{f}_{\infty}^{2} \\
0 & 0 & 0 & 0 & 0 & I \otimes \overline{\mathfrak{f}}_{\infty}^{2} \\
0 & 0 & 0 & 0 & 0 & \overline{\mathfrak{f}}_{\infty}^{1} \otimes I \\
0 & 0 & 0 & 0 & 0 & 0
\end{array}\right)
$$

with $\Gamma=\left(\overline{\mathfrak{f}}_{0}^{1} \circ \mathfrak{f}_{\infty}^{1}\right) \otimes\left(\overline{\mathfrak{f}}_{0}^{2} \circ \mathfrak{f}_{\infty}^{2}\right)+\theta^{1} \otimes \bar{\theta}^{2}+\bar{\theta}^{1} \otimes \theta^{1}, \Phi=\overline{\mathfrak{f}}_{\infty}^{1} \otimes \mathfrak{f}_{0}^{2}+\mathfrak{f}_{\infty}^{1} \otimes \overline{\mathfrak{f}}_{0}^{2}$ and $\Psi=\mathfrak{f}_{0}^{1} \otimes \overline{\mathfrak{f}}_{\infty}^{2}+\overline{\mathfrak{f}}_{0}^{1} \otimes \mathfrak{f}_{\infty}^{2}$.

The dimension of the $\mathbb{F}$-vector space $H_{*}\left(B, d_{B}\right)$ only depends on the rank of the kernel and the cokernel of the matrix $d_{B}$. Define a pair of matrices $M_{1}$ and $M_{2}$ to be equivalent if $\operatorname{Ker}\left(M_{1}\right) \simeq \operatorname{Ker}\left(M_{2}\right)$ and $\operatorname{Coker}\left(M_{1}\right) \simeq \operatorname{Coker}\left(M_{2}\right)$. For a matrix $M$, let

$$
l(M):=\operatorname{Ker}(M) \oplus \operatorname{Coker}(M) \quad \text { and } \quad i(M):=\operatorname{rnk}(l(M)) .
$$

If $M_{1}$ and $M_{2}$ are equivalent matrices then $l\left(M_{1}\right) \simeq l\left(M_{2}\right)$ and $i\left(M_{1}\right)=i\left(M_{2}\right)$.

We make a change of basis for $\mathbb{E}_{2}$ which is given by the matrix

$$
\left(\begin{array}{ccc}
\tau_{0}\left(K_{1}\right) \otimes \tau_{1}\left(K_{2}\right) & 0 & 0 \\
0 & \tau_{1}\left(K_{1}\right) \otimes \tau_{0}\left(K_{2}\right) & 0 \\
0 & 0 & \tau_{0}\left(K_{1}\right) \otimes \tau_{0}\left(K_{2}\right)
\end{array}\right) .
$$

The matrix $d_{B}$ is thus equivalent to the matrix

$$
d_{B}^{\prime}=\left(\begin{array}{cccccc}
0 & \mathfrak{f}_{0}^{1} \otimes I & I \otimes \mathfrak{f}_{0}^{2} & \theta^{1} \tau_{0}^{1} \otimes \tau_{\infty}^{2} \mathfrak{f}_{0}^{2} & \tau_{\infty}^{1} \mathfrak{f}_{0}^{1} \otimes \theta^{2} \tau_{0}^{2} & \Gamma \\
0 & 0 & 0 & \Phi & \tau_{1}^{1} \otimes \mathfrak{f}_{0}^{2} \tau_{1}^{2} \mathfrak{f}_{\infty}^{2} & \mathfrak{f}_{\infty}^{1} \tau_{0}^{1} \otimes \bar{\theta}^{2} \tau_{0}^{2} \\
0 & 0 & 0 & \mathfrak{f}_{0}^{1} \tau_{1}^{1} \mathfrak{f}_{\infty}^{1} \otimes \tau_{1}^{1} & \Psi & \bar{\theta} \tau_{0}^{1} \otimes \mathfrak{f}_{\infty}^{2} \tau_{0}^{2} \\
0 & 0 & 0 & 0 & 0 & I \otimes \mathfrak{f}_{\infty}^{2} \\
0 & 0 & 0 & 0 & 0 & \mathfrak{f}_{\infty}^{1} \otimes I \\
0 & 0 & 0 & 0 & 0 & 0
\end{array}\right)
$$


with

$$
\begin{aligned}
& \Phi=\tau_{1}^{1} \mathfrak{f}_{\infty}^{1} \otimes \mathfrak{f}_{0}^{2} \tau_{1}^{2}+\mathfrak{f}_{\infty}^{1} \tau_{0}^{1} \otimes \tau_{\infty}^{2} \mathfrak{f}_{0}^{2}, \\
& \Psi=\mathfrak{f}_{0}^{1} \tau_{1}^{1} \otimes \tau_{1}^{2} \mathfrak{f}_{\infty}^{2}+\tau_{\infty}^{1} \mathfrak{f}_{0}^{1} \otimes \mathfrak{f}_{\infty}^{2} \tau_{0}^{2}, \\
& \Gamma=\tau_{\infty}^{1} \mathfrak{f}_{0}^{1} \overline{\mathfrak{f}}_{\infty}^{1} \otimes \tau_{\infty}^{2} \mathfrak{f}_{0}^{2} \overline{\mathfrak{f}}_{\infty}^{2}+\theta^{1} \tau_{0}^{1} \otimes \bar{\theta}^{2} \tau_{0}^{2}+\bar{\theta}^{1} \tau_{0}^{1} \otimes \theta^{2} \tau_{0}^{2} .
\end{aligned}
$$

Let us use the decompositions of (5) for $K_{1}$ and $K_{2}$ to obtain a $24 \times 24$ block decomposition of $d_{B}^{\prime}$. Moreover, following the discussion at the end of Section 3.3 we may assume that, in the corresponding decompositions,

$$
\theta^{i}=\left(\begin{array}{ll}
0 & I \\
0 & 0
\end{array}\right) \quad \text { and } \quad \tau_{\infty}^{i} \bar{\theta}^{i} \tau_{0}^{i}=\left(\begin{array}{cc}
M^{i} & I \\
P^{i} M^{i} & P^{i}
\end{array}\right)
$$

Each entry in the above $6 \times 6$ decomposition for $d_{\mathbb{B}}^{\prime}$ corresponds to a $4 \times 4$ submatrix of the aforementioned $24 \times 24$ decomposition. For instance, the $(1,4)$ entry $\theta^{1} \tau_{0}^{1} \otimes \tau_{\infty}^{2} \mathfrak{f}_{0}^{2}$ corresponds to

$$
\begin{aligned}
\left(\begin{array}{ll}
0 & I \\
0 & 0
\end{array}\right)\left(\begin{array}{ll}
A_{0}^{1} & B_{0}^{1} \\
C_{0}^{1} & D_{0}^{1}
\end{array}\right) \otimes\left(\begin{array}{ll}
A_{\infty}^{2} & B_{\infty}^{2} \\
C_{\infty}^{2} & D_{\infty}^{2}
\end{array}\right)\left(\begin{array}{ll}
0 & 0 \\
I & 0
\end{array}\right) & =\left(\begin{array}{cc}
C_{0}^{1} & D_{0}^{1} \\
0 & 0
\end{array}\right) \otimes\left(\begin{array}{cc}
B_{\infty}^{2} & 0 \\
D_{\infty}^{2} & 0
\end{array}\right) \\
& =\left(\begin{array}{ccccc}
C_{0}^{1} \otimes B_{\infty}^{2} & 0 & D_{0}^{1} \otimes B_{\infty}^{2} & 0 \\
C_{0}^{1} \otimes D_{\infty}^{2} & 0 & D_{0}^{1} \otimes D_{\infty}^{2} & 0 \\
0 & 0 & 0 & 0 \\
0 & 0 & 0 & 0
\end{array}\right) .
\end{aligned}
$$

For another instance, the $(3,5)$ entry corresponds to

$$
\begin{aligned}
\left(\begin{array}{ccccc}
0 & 0 & 0 & 0 \\
0 & 0 & 0 & 0 \\
A_{1}^{1} \otimes B_{1}^{2} & 0 & B_{1}^{1} \otimes B_{1}^{2} & 0 \\
A_{1}^{1} \otimes D_{1}^{2} & 0 & B_{1}^{1} \otimes D_{1}^{2} & 0
\end{array}\right)+ & \left(\begin{array}{cccc}
0 & 0 & 0 & 0 \\
B_{\infty}^{1} \otimes A_{0}^{2} & B_{\infty}^{1} \otimes B_{0}^{2} & 0 & 0 \\
0 & 0 & 0 & 0 \\
D_{\infty}^{1} \otimes A_{0}^{2} & D_{\infty}^{1} \otimes B_{0}^{2} & 0 & 0
\end{array}\right) . \\
& =\left(\begin{array}{ccccc}
0 & 0 & 0 & 0 \\
B_{\infty}^{1} \otimes A_{0}^{2} & B_{\infty}^{1} \otimes B_{0}^{2} & 0 & 0 \\
A_{1}^{1} \otimes B_{1}^{2} & 0 & B_{1}^{1} \otimes B_{1}^{2} & 0 \\
A_{1}^{1} \otimes D_{1}^{2}+D_{\infty}^{1} \otimes A_{0}^{2} & D_{\infty}^{1} \otimes B_{0}^{2} & B_{1}^{1} \otimes D_{1}^{2} & 0
\end{array}\right) .
\end{aligned}
$$

The aforementioned $24 \times 24$ decomposition includes identity matrices as the entries determined by the following block coordinates:
$(2,9)$,
$(3,5)$,
$(4,6)$
$(14,21)$
$(16,23)$ and
$(20,22)$.

We use the above six identity matrices for cancellation to obtain an equivalent matrix $d=\left(\begin{array}{ll}0 & D \\ 0 & 0\end{array}\right)$ over $\mathbb{B}_{1} \oplus \mathbb{B}_{2}$, where $\mathbb{A}_{\bullet \star}=\mathbb{A}_{\bullet}\left(K_{1}\right) \otimes \mathbb{A}_{\star}\left(K_{2}\right)$ and 


$$
\begin{aligned}
& \mathbb{B}_{1}=\mathbb{A}_{11} \oplus \mathbb{A}_{\infty 1} \oplus \mathbb{A}_{\infty 0} \oplus \mathbb{A}_{1 \infty} \oplus \mathbb{A}_{0 \infty} \oplus \mathbb{A}_{00}, \\
& \mathbb{B}_{2}=\mathbb{A}_{\infty 0} \oplus \mathbb{A}_{10} \oplus \mathbb{A}_{\infty \infty} \oplus \mathbb{A}_{0 \infty} \oplus \mathbb{A}_{01} \oplus \mathbb{A}_{11} .
\end{aligned}
$$

Rearrange the rows and the columns of the matrix $D$ so that $D$ corresponds to the rows $11,7,8,10,12,1$ and the columns $19,13,15,17,18,24$ in the above $24 \times 24$ decomposition to obtain the matrix

$$
\left(\begin{array}{cccccc}
B_{1}^{1} \otimes B_{1}^{2} & B_{1}^{1} \otimes A_{1}^{2} & 0 & A_{1}^{1} \otimes B_{1}^{2} & 0 & 0 \\
0 & A_{0}^{1} \otimes B_{\infty}^{2} & B_{0}^{1} \otimes B_{\infty}^{2} & 0 & 0 & B_{0}^{1} \otimes\left(A_{\infty}^{2}+B_{\infty}^{2} P^{2}\right) \\
D_{1}^{1} \otimes B_{1}^{2} & D_{1}^{1} \otimes A_{1}^{2}+A_{0}^{1} \otimes D_{\infty}^{2} & B_{0}^{1} \otimes D_{\infty}^{2} & C_{1}^{1} \otimes B_{1}^{2} & 0 & B_{0}^{1} \otimes\left(C_{\infty}^{2}+D_{\infty}^{2} P^{2}\right) \\
0 & 0 & 0 & B_{\infty}^{1} \otimes A_{0}^{2} & B_{\infty}^{1} \otimes B_{0}^{2}\left(A_{\infty}^{1}+B_{\infty}^{1} P^{1}\right) \otimes B_{0}^{2} \\
B_{1}^{1} \otimes D_{1}^{2} & B_{1}^{1} \otimes C_{1}^{2} & 0 & D_{\infty}^{1} \otimes A_{0}^{2}+A_{1}^{1} \otimes D_{1}^{2} & D_{\infty}^{1} \otimes B_{0}^{2}\left(C_{\infty}^{1}+D_{\infty}^{1} P^{1}\right) \otimes B_{0}^{2} \\
0 & C_{0}^{1} \otimes B_{\infty}^{2} & D_{0}^{1} \otimes B_{\infty}^{2} & B_{\infty}^{1} \otimes C_{0}^{2} & B_{\infty}^{1} \otimes D_{0}^{2} & \Gamma
\end{array}\right)
$$

with $\Gamma=B_{\infty}^{1} B_{1}^{1} B_{0}^{1} \otimes B_{\infty}^{2} B_{1}^{2} B_{0}^{2}+\left(A_{\infty}^{1}+B_{\infty}^{1} P^{1}\right) \otimes D_{0}^{2}+D_{0}^{1} \otimes\left(A_{\infty}^{2}+B_{\infty}^{2} P^{2}\right)$.

This matrix is in turn equivalent to the matrix $\mathfrak{D}=\mathfrak{D}\left(K_{1}, K_{2}\right)$ below, which is obtained by adding $I \otimes P^{2}$ times the third column and $P^{1} \otimes I$ times the fifth column to the last column of the above matrix:

$$
\mathfrak{D}=\left(\begin{array}{cccccc}
B_{1}^{1} \otimes B_{1}^{2} & C_{1}^{1} \otimes A_{1}^{2} & 0 & A_{1}^{1} \otimes B_{1}^{2} & 0 & 0 \\
0 & A_{0}^{1} \otimes B_{\infty}^{2} & B_{0}^{1} \otimes B_{\infty}^{2} & 0 & 0 & B_{0}^{1} \otimes A_{\infty}^{2} \\
D_{1}^{1} \otimes B_{1}^{2} & D_{1}^{1} \otimes A_{1}^{2}+A_{0}^{1} \otimes D_{\infty}^{2} & B_{0}^{1} \otimes D_{\infty}^{2} & C_{1}^{1} \otimes B_{1}^{2} & 0 & B_{0}^{1} \otimes C_{\infty}^{2} \\
0 & 0 & 0 & B_{\infty}^{1} \otimes A_{0}^{2} & B_{\infty}^{1} \otimes B_{0}^{2} & A_{\infty}^{1} \otimes B_{0}^{2} \\
B_{1}^{1} \otimes D_{1}^{2} & B_{1}^{1} \otimes C_{1}^{2} & 0 & D_{\infty}^{1} \otimes A_{0}^{2}+A_{1}^{1} \otimes D_{1}^{2} & D_{\infty}^{1} \otimes B_{0}^{2} & C_{\infty}^{1} \otimes B_{0}^{2} \\
0 & C_{0}^{1} \otimes B_{\infty}^{2} & D_{0}^{1} \otimes B_{\infty}^{2} & B_{\infty}^{1} \otimes C_{0}^{2} & B_{\infty}^{1} \otimes D_{0}^{2} & \Psi
\end{array}\right),
$$

where $\Psi=A_{\infty}^{1} \otimes D_{0}^{2}+D_{0}^{1} \otimes A_{\infty}^{2}+X^{1} \otimes X^{2}$ and $X^{i}=X\left(K_{i}\right)=B_{\infty}^{i} B_{1}^{i} B_{0}^{i}$ for $i=1,2$.

Combining Proposition 5.3 with the above observations, we find:

Proposition 5.5 Let $K_{i} \subset Y_{i}, i=1,2$, denote null-homologous knots and $Y\left(K_{1}, K_{2}\right)$ denote the three-manifold obtained by splicing the complement of $K_{1}$ with the complement of $K_{2}$. With the above definition of $\mathfrak{D}\left(K_{1}, K_{2}\right)$,

$$
\widehat{\mathrm{HF}}\left(Y\left(K_{1}, K_{2}\right), \mathbb{F}\right) \simeq l\left(\mathfrak{D}\left(K_{1}, K_{2}\right)\right) .
$$

Corollary 5.6 The splicing formula of Proposition 5.3 is independent of the choice of extensions $\theta^{i}$ and $\bar{\theta}^{i}$.

Proof The fact that the matrices $P^{i}$ and $M^{i}$ do not appear in the matrix $\mathfrak{D}\left(K_{1}, K_{2}\right)$ implies that the choice of the extensions $\theta^{i}, \bar{\theta}^{i}: \mathbb{H}_{0}^{i} \rightarrow \mathbb{H}_{\infty}^{i}$ does not change the rank of the homology group in the splicing formula of Proposition 5.3 or Theorem 1.1. 
With the above corollary in place, the proof of Theorem 1.1 is now complete.

Definition 5.7 For a pair of knots $K_{i} \subset Y_{i}, i=1,2$, define

$$
\begin{aligned}
\chi\left(K_{1}, K_{2}\right):=\left(h_{1}\left(K_{1}\right)-h_{\infty}\left(K_{1}\right)\right)\left(h_{1}\left(K_{2}\right)-h_{\infty}\left(K_{2}\right)\right) & \\
& -\left(h_{1}\left(K_{1}\right)-h_{0}\left(K_{1}\right)\right)\left(h_{1}\left(K_{2}\right)-h_{0}\left(K_{2}\right)\right) .
\end{aligned}
$$

Note that $\chi\left(K_{1}, K_{2}\right)$ is in fact the difference between the ranks of $\mathbb{B}_{1}=\mathbb{B}_{1}\left(K_{1}, K_{2}\right)$ and $\mathbb{B}_{2}=\mathbb{B}_{2}\left(K_{1}, K_{2}\right)$. In the corresponding $\mathbb{Z} / 2 \mathbb{Z}$-grading on $\mathbb{B}_{1} \oplus \mathbb{B}_{2}, \chi\left(K_{1}, K_{2}\right)$ is thus the Euler characteristic of the chain complex $\left(\mathbb{B}_{1} \oplus \mathbb{B}_{2}, d\right)$.

Corollary 5.8 With the above notation fixed,

$$
\operatorname{rnk}\left(\widehat{\mathrm{HF}}\left(Y\left(K_{1}, K_{2}\right)\right)\right) \geq\left|\chi\left(K_{1}, K_{2}\right)\right| .
$$

Proof It is enough to note that

$$
\chi\left(K_{1}, K_{2}\right)=\operatorname{rnk}\left(\operatorname{Ker}\left(\mathfrak{D}\left(K_{1}, K_{2}\right)\right)\right)-\operatorname{rnk}\left(\operatorname{Coker}\left(\mathfrak{D}\left(K_{1}, K_{2}\right)\right)\right) .
$$

Consider the matrices

$$
\begin{aligned}
P_{L} & =\left(\begin{array}{cccccc}
I \otimes A_{1}^{2} & 0 & 0 & 0 & I \otimes B_{1}^{2} & 0 \\
0 & I \otimes A_{\infty}^{2} & I \otimes B_{\infty}^{2} & 0 & 0 & 0 \\
0 & I \otimes C_{\infty}^{2} & I \otimes D_{\infty}^{2} & 0 & 0 & 0 \\
0 & 0 & 0 & I \otimes A_{0}^{2} & 0 & I \otimes B_{0}^{2} \\
I \otimes C_{1}^{2} & 0 & 0 & 0 & I \otimes D_{1}^{2} & 0 \\
0 & 0 & 0 & I \otimes C_{0}^{2} & 0 & I \otimes D_{0}^{2}
\end{array}\right), \\
P_{R} & =\left(\begin{array}{cccccc}
D_{1}^{1} \otimes I & 0 & 0 & C_{1}^{1} \otimes I & 0 & 0 \\
0 & A_{0}^{1} \otimes I & B_{0}^{1} \otimes I & 0 & 0 & 0 \\
0 & C_{0}^{1} \otimes I & D_{0}^{1} \otimes I & 0 & 0 & 0 \\
B_{1}^{1} \otimes I & 0 & 0 & A_{1}^{1} \otimes I & 0 & 0 \\
0 & 0 & 0 & 0 & D_{\infty}^{1} \otimes I & C_{\infty}^{1} \otimes I \\
0 & 0 & 0 & 0 & B_{\infty}^{1} \otimes I & A_{\infty}^{1} \otimes I
\end{array}\right) .
\end{aligned}
$$

Since $P_{R}^{2}=P_{L}^{2}=\mathrm{Id}$, both $P_{R}$ and $P_{L}$ are invertible and $\mathfrak{D}\left(K_{1}, K_{2}\right)$ is equivalent to $\mathfrak{D}^{\prime}\left(K_{1}, K_{2}\right)=P_{L} \mathfrak{D}\left(K_{1}, K_{2}\right) P_{R}$. The matrix $\mathfrak{D}^{\prime}\left(K_{1}, K_{2}\right)$ has the block presentation

$$
\left(\begin{array}{cccccc}
D_{\infty}^{1} B_{1}^{1} \otimes B_{1}^{2} A_{0}^{2} & B_{1}^{1} A_{0}^{1} \otimes I & B_{1}^{1} B_{0}^{1} \otimes I & D_{\infty}^{1} A_{1}^{1} \otimes B_{1}^{2} A_{0}^{2} I \otimes B_{1}^{2} B_{0}^{2} & 0 \\
I \otimes B_{\infty}^{2} B_{1}^{2} & D_{1}^{1} A_{0}^{1} \otimes B_{\infty}^{2} A_{1}^{2} & D_{1}^{1} B_{0}^{1} \otimes B_{\infty}^{2} A_{1}^{2} & 0 & B_{0}^{1} B_{\infty}^{1} \otimes I & B_{0}^{1} A_{\infty}^{1} \otimes I \\
I \otimes D_{\infty}^{2} B_{1}^{2} & \Psi_{1} & D_{1}^{1} B_{0}^{1} \otimes D_{\infty}^{2} A_{1}^{2} & 0 & 0 & 0 \\
B_{\infty}^{1} B_{1}^{1} \otimes I & 0 & I \otimes B_{0}^{2} B_{\infty}^{2} & B_{\infty}^{1} A_{1}^{1} \otimes I & \Gamma_{1} & \Gamma_{2} \\
D_{\infty}^{1} B_{1}^{1} \otimes D_{1}^{2} A_{0}^{2} & 0 & 0 & \Psi_{2} & I \otimes D_{1}^{2} B_{0}^{2} & 0 \\
0 & 0 & I \otimes D_{0}^{2} B_{\infty}^{2} & 0 & \Gamma_{3} & \Gamma_{4}
\end{array}\right)
$$


with

$$
\begin{aligned}
\Psi_{1}=I \otimes I+D_{1}^{1} A_{0}^{1} \otimes D_{\infty}^{2} A_{1}^{2}, & \Gamma_{1}=D_{0}^{1} B_{\infty}^{1} \otimes B_{0}^{2} A_{\infty}^{2}+X^{1} B_{\infty}^{1} \otimes B_{0}^{2} X^{2}, \\
\Psi_{2}=I \otimes I+D_{\infty}^{1} A_{1}^{1} \otimes D_{1}^{2} A_{0}^{2}, & \Gamma_{2}=D_{0}^{1} A_{\infty}^{1} \otimes B_{0}^{2} A_{\infty}^{2}+X^{1} A_{\infty}^{1} \otimes B_{0}^{2} X^{2}, \\
& \Gamma_{3}=D_{0}^{1} B_{\infty}^{1} \otimes D_{0}^{2} A_{\infty}^{2}+X^{1} B_{\infty}^{1} \otimes D_{0}^{2} X^{2}, \\
& \Gamma_{4}=I \otimes I+D_{0}^{1} A_{\infty}^{1} \otimes D_{0}^{2} A_{\infty}^{2}+X^{1} A_{\infty}^{1} \otimes D_{0}^{2} X^{2},
\end{aligned}
$$

and is easier to use in actual computations. Note that

$$
l\left(\mathfrak{D}^{\prime}\left(K_{1}, K_{2}\right)\right) \simeq l\left(\mathfrak{D}\left(K_{1}, K_{2}\right)\right) \simeq \widehat{\mathrm{HF}}\left(Y\left(K_{1}, K_{2}\right), \mathbb{F}\right) .
$$

\section{Splicing with the trefoil}

\subsection{The maps $\mathfrak{f}_{\bullet}$ and $\overline{\mathfrak{f}}_{\bullet}$ for the trefoils}

Let us now consider the case of the right-handed trefoil, which will be denoted by $R$. Thus, $h_{\infty}(R)=h_{1}(R)=3$ and $h_{0}(R)=4$. Moreover, $y_{\infty}(R)=y_{1}(R)=1$, while $y_{0}(R)=2$ (see Eftekhary [3, Section 5]). Since $\mathbb{H}_{\bullet}(R, i)=\mathbb{F}$ for $\bullet=1, \infty, i=0, \pm 1$, the maps $\tau_{1}(R)$ and $\tau_{\infty}(R)$ are forced and we only need to determine $\tau_{0}(R)$.

The decompositions of $\mathbb{H}_{\infty}(R)=\mathbb{H}_{1}(R)=\mathbb{F}^{3}$ according to relative $\operatorname{Spin}^{c}$ classes give

$$
\mathbb{H}_{1}(R)=\langle a, b, c\rangle_{\mathbb{F}} \quad \text { and } \quad \mathbb{H}_{\infty}(R)=\left\langle a^{\prime}, b^{\prime}, c^{\prime}\right\rangle_{\mathbb{F}},
$$

where $a, a^{\prime}$ are generators in relative $\operatorname{Spin}^{c}$ class $-1, b, b^{\prime}$ are generators in relative $\operatorname{Spin}^{c}$ class 0 and $c, c^{\prime}$ are generators in relative $\operatorname{Spin}^{c}$ class +1 . The homomorphisms $\mathfrak{f}_{0}(R)$ and $\overline{\mathfrak{f}}_{0}(R)$ have the following block forms in the corresponding basis:

$$
\mathfrak{f}_{0}(R)=\left(\begin{array}{ccc}
\alpha & 0 & 0 \\
0 & \beta & 0 \\
0 & 0 & \gamma
\end{array}\right) \quad \text { and } \quad \overline{\mathfrak{f}}_{0}(R)=\left(\begin{array}{ccc}
\gamma & 0 & 0 \\
0 & \beta & 0 \\
0 & 0 & \alpha
\end{array}\right) \text {. }
$$

From (7) we know that the ranks of $\mathfrak{f}_{0}(R)$ and $\overline{\mathfrak{f}}_{0}(R)$ are equal to 1 , ie precisely one of $\alpha, \beta$ and $\gamma$ is equal to 1 and the other two are zero. Moreover, the rank of $\mathfrak{f}_{0}(R)+\overline{\mathfrak{f}}_{0}(R)$ is 2 , ie precisely two of $\alpha+\gamma, \alpha+\gamma, 2 \beta$ are non-zero. Since the coefficient ring is $\mathbb{F}, 2 b$ is automatically zero. Thus, $\alpha=1$ and $\beta=\gamma=0$, or $\gamma=1$ and $\alpha=\beta=0$.

The generator $a$ of $\mathbb{H}_{1}(R)$ is not in the image of $\mathfrak{f}_{\infty}(R)$, since $\mathfrak{f}_{\infty}(R,-1)$ is trivial. Hence $a$ is not in the kernel of $\mathfrak{f}_{0}(R,-1)$. Thus, from the above two possibilities the former is the case, ie in (18) we get $\alpha=1$ and $\beta=\gamma=0$. 
The rank of $\overline{\mathfrak{f}}_{\infty}(R)$ is equal to 2 according to (7). Moreover, $\langle a, b\rangle_{\mathbb{F}}$ is already in the image of $\overline{\mathfrak{f}}_{0}(R)$. Thus, $\overline{\mathfrak{f}}_{0}(R)$ is surjective onto $\mathbb{H}_{1}(R,-1) \oplus \mathbb{H}_{1}(R, 0)$. Let us use a basis $a^{\prime \prime}, b^{\prime \prime}$ for $\mathbb{H}_{0}\left(R,-\frac{1}{2}\right)$ which contains some pre-image $a^{\prime \prime}$ of $a$ under $\overline{\mathfrak{f}}_{\infty}$ and an element $b^{\prime \prime}$ in the kernel of $\overline{\mathfrak{f}}_{\infty}$. Use the dual basis $\tau_{0}\left(b^{\prime \prime}\right), \tau_{0}\left(a^{\prime \prime}\right)$ for $\mathbb{H}_{0}\left(R, \frac{1}{2}\right)$. The basis $\left\{a^{\prime \prime}, b^{\prime \prime}, \tau_{0}\left(b^{\prime \prime}\right), \tau_{0}\left(a^{\prime \prime}\right)\right\}$ for $\mathbb{H}_{0}(R)$ is thus invariant under $\tau_{0}=\tau_{0}(R)$. Correspondingly, we get

$$
\overline{\mathfrak{f}}_{\infty}(R)=\left(\begin{array}{cccc}
1 & 0 & 0 & 0 \\
0 & 0 & x & y \\
0 & 0 & 0 & 0
\end{array}\right) \quad \text { and } \quad \mathfrak{f}_{\infty}(R)=\left(\begin{array}{llll}
0 & 0 & 0 & 0 \\
y & x & 0 & 0 \\
0 & 0 & 0 & 1
\end{array}\right) .
$$

If $x=0$ then $y=1$, since the rank of $\mathfrak{f}_{\infty}(R)$ is equal to 2 . The rank of $\mathfrak{f}_{\infty}(R)+\overline{\mathfrak{f}}_{\infty}(R)$ is then equal to 2; on the other hand, (7) implies that this rank is 3 , a contradiction. The contradiction implies that $x=1$. Replacing $a^{\prime \prime}$ with $a^{\prime \prime}-y b^{\prime \prime}$, we obtain the presentation of $\mathfrak{f}_{\infty}(R)$ and $\overline{\mathfrak{f}}_{\infty}(R)$ in a new basis for $\mathbb{H}_{0}(R)$ (which is still invariant under the involution $\tau_{0}(R)$ ) corresponding to the values $x=1$ and $y=0$ in (19). From here, by taking into account the fact that the map $\theta(R)$ increases the $\operatorname{Spin}^{c}$ grading by $\frac{1}{2}$ while $\bar{\theta}(R)$ decreases the $\operatorname{Spin}^{c}$ grading by $\frac{1}{2}$,

$$
\theta(R)=\left(\begin{array}{llll}
0 & 0 & 0 & 0 \\
1 & 0 & 0 & 0 \\
0 & 0 & 1 & 0
\end{array}\right) \text { and } \bar{\theta}(R)=\left(\begin{array}{llll}
0 & 1 & 0 & 0 \\
0 & 0 & 0 & 1 \\
0 & 0 & 0 & 0
\end{array}\right) .
$$

The above computations imply that $a_{0}(R)=1$ while $a_{1}(R)=a_{\infty}(R)=2$. Moreover, we may take

$$
\begin{gathered}
A_{0}(R)=D_{0}(R)=\left(\begin{array}{ll}
0 & 0 \\
0 & 0
\end{array}\right), \quad B_{0}(R)=C_{0}(R)=\left(\begin{array}{ll}
0 & 1 \\
1 & 0
\end{array}\right), \\
A_{1}(R)=D_{\infty}(R)=(0), \quad D_{1}(R)=A_{\infty}(R)=\left(\begin{array}{ll}
0 & 0 \\
0 & 1
\end{array}\right), \\
C_{1}(R)=B_{\infty}(R)=B_{1}^{T}(R)=C_{\infty}^{T}(R)=\left(\begin{array}{l}
1 \\
0
\end{array}\right) .
\end{gathered}
$$

For the left-handed trefoil, a similar argument may be used for the computation, which is sketched below. The rank of $\mathfrak{f}_{0}(L)$ is 2 and the rank of $\mathfrak{f}_{\infty}(L)$ is 3 . The latter implies that the rank of $\mathfrak{f}_{\infty}(L, 1)$ is 1 , the rank of $\mathfrak{f}_{\infty}(L, 0)$ is 2 and the rank of $\mathfrak{f}_{\infty}(L,-1)$ is zero. Correspondingly, the ranks of $\mathfrak{f}_{0}(L, 1), \mathfrak{f}_{0}(L, 0)$ and $\mathfrak{f}_{0}(L,-1)$ are equal to 0,1 and 1 , respectively. If the images of $\mathfrak{f}_{\infty}(L, 0)$ and $\overline{\mathfrak{f}}_{\infty}(L, 0)$ are identical, the maps $\mathfrak{f}_{0}(L, 0)$ and $\overline{\mathfrak{f}}_{0}(L, 0)$ are forced to be identical, since $\mathbb{H}_{\infty}(L, 0)$ is 1-dimensional. In particular, $\mathfrak{f}_{0}(L, 0)+\overline{\mathfrak{f}}_{0}(L, 0)$ is trivial. Hence the rank of $\mathfrak{f}_{0}(L)+\overline{\mathfrak{f}}_{0}(L)$ is at most 2 , which is in contradiction with $y_{0}(L)=2$. The 2 -dimensional subspaces $\operatorname{Im}\left(\mathfrak{f}_{\infty}(L, 0)\right)$ and $\operatorname{Im}\left(\overline{\mathfrak{f}}_{\infty}(L, 0)\right)$ of $\mathbb{H}_{1}(L, 0)$ are thus different. From here, their 
intersection is 1 -dimensional and is generated by some $\tau_{1}(L)$-invariant element $\mathfrak{f}_{\infty}(b)$ with $b \in \mathbb{H}_{0}\left(L,-\frac{1}{2}\right)$.

Let $a \in \mathbb{H}_{0}\left(L, \frac{1}{2}\right)$ denote the unique non-trivial vector in the kernel of $\mathfrak{f}_{\infty}(L)$. Let us first assume that $b=\tau_{0}(a)$. Complete $a$ to a basis $(a, c)$ for $\mathbb{H}_{0}\left(L, \frac{1}{2}\right)$. Then

$$
\left\{a, c, \tau_{0}(a), \tau_{0}(c)\right\}
$$

is an ordered basis for $\mathbb{H}_{0}(L)$. Correspondingly, we obtain the basis

$$
\left\{\mathfrak{f}_{\infty}(c), \overline{\mathfrak{f}}_{\infty}(c), \mathfrak{f}_{\infty}\left(\tau_{0}(a)\right), \mathfrak{f}_{\infty}\left(\tau_{0}(c)\right), \tau_{1}\left(\mathfrak{f}_{\infty}(c)\right)\right\}
$$

for $\mathbb{H}_{1}(L)$ and the matrices $\mathfrak{f}_{\infty}(L)$ and $\overline{\mathfrak{f}}_{\infty}(L)$ take the following forms, respectively:

$$
\mathfrak{f}_{\infty}(L)=\left(\begin{array}{cccc}
0 & 1 & 0 & 0 \\
0 & 0 & 0 & 0 \\
0 & 0 & 1 & 0 \\
0 & 0 & 0 & 1 \\
0 & 0 & 0 & 0
\end{array}\right) \text { and } \overline{\mathfrak{f}}_{\infty}(L)=\left(\begin{array}{llll}
0 & 0 & 0 & 0 \\
0 & 1 & 0 & 0 \\
1 & 0 & 0 & 0 \\
0 & 0 & 0 & 0 \\
0 & 0 & 0 & 1
\end{array}\right) .
$$

In particular, the matrix

$$
\mathfrak{f}_{\infty}(L)+\overline{\mathfrak{f}}_{\infty}(L)=\left(\begin{array}{llll}
0 & 1 & 0 & 0 \\
0 & 1 & 0 & 0 \\
1 & 0 & 1 & 0 \\
0 & 0 & 0 & 1 \\
0 & 0 & 0 & 1
\end{array}\right)
$$

is a matrix of rank 3 , while we should have

$$
\operatorname{rnk}\left(\mathfrak{f}_{\infty}(L)+\overline{\mathfrak{f}}_{\infty}(L)\right)=\frac{1}{2}\left(h_{0}(L)+h_{1}(L)-y_{\infty}(L)\right)=4 .
$$

This contradiction implies that $b$ is different from $\tau_{0}(a)$, so we may take $\left(\tau_{0}(a), b\right)$ as a basis for $\mathbb{H}_{0}\left(L, \frac{1}{2}\right)$. Correspondingly, we obtain the basis

$$
\left\{\tau_{0}(a), b, \tau_{0}(b), a\right\}
$$

for $\mathbb{H}_{0}(L)$. As a basis for $\mathbb{H}_{1}(L, 0)$ we obtain the three vectors $\mathfrak{f}_{\infty}(a), \overline{\mathfrak{f}}_{\infty}(a)$ and $\overline{\mathfrak{f}}_{\infty}(b)$. This basis is completed to the (ordered) basis for $\mathbb{H}_{1}(L)$

$$
\left\{\mathfrak{f}_{\infty}(a), \overline{\mathfrak{f}}_{\infty}(b), \mathfrak{f}_{\infty}(b), \overline{\mathfrak{f}}_{\infty}(a), \tau_{1}\left(\mathfrak{f}_{\infty}(b)\right)\right\} .
$$

Finally, we choose the following basis for $\mathbb{H}_{\infty}(L)$ :

$$
\left\{\overline{\mathfrak{f}}_{0}\left(\mathfrak{f}_{\infty}(b)\right), \mathfrak{f}_{0}\left(\overline{\mathfrak{f}}_{\infty}(a)\right), \tau_{\infty}\left(\overline{\mathfrak{f}}_{0}\left(\mathfrak{f}_{\infty}(b)\right)\right)\right\} .
$$


In these bases, we may compute

$$
\mathfrak{f}_{\infty}(L)=\left(\begin{array}{llll}
1 & 0 & 0 & 0 \\
0 & 1 & 0 & 0 \\
0 & 0 & 1 & 0 \\
0 & 0 & 0 & 0
\end{array}\right) \quad \text { and } \quad \mathfrak{f}_{0}(L)=\left(\begin{array}{lllll}
0 & 0 & 0 & 0 & 0 \\
0 & 0 & 0 & 1 & 0 \\
0 & 0 & 0 & 0 & 1
\end{array}\right) .
$$

Moreover, after re-ordering the elements of the above bases, we find the presentations

$$
\begin{gathered}
D_{0}(L)=A_{\infty}(L)=0, \quad A_{1}(L)=\left(\begin{array}{ll}
0 & 0 \\
0 & 0
\end{array}\right), \quad B_{1}(L)=C_{1}^{T}(L)=\left(\begin{array}{lll}
1 & 0 & 0 \\
0 & 0 & 1
\end{array}\right), \\
A_{0}(L)=\left(\begin{array}{lll}
0 & 0 & 0 \\
0 & 0 & 1 \\
0 & 1 & 0
\end{array}\right), \quad D_{1}(L)=\left(\begin{array}{lll}
0 & 0 & 0 \\
0 & 1 & 0 \\
0 & 0 & 0
\end{array}\right), \quad D_{\infty}(L)=\left(\begin{array}{ll}
1 & 0 \\
0 & 0
\end{array}\right), \\
B_{0}(L)=C_{0}^{T}(L)=\left(\begin{array}{l}
1 \\
0 \\
0
\end{array}\right) \quad \text { and } \quad B_{\infty}(L)=C_{\infty}^{T}(L)=\left(\begin{array}{ll}
0 & 1
\end{array}\right) .
\end{gathered}
$$

\subsection{Splicing a knot complement with the complement of a trefoil}

For a knot $K \subset Y$, let $Y(R, K)$ denote the three-manifold obtained by splicing the complement of $K \subset Y$ with the complements of the right-handed trefoil. We study the rank $r_{r}(K)$ of $\widehat{\mathrm{HF}}(Y(R, K))$ in this subsection. With the notation of Section 5.3, $r_{r}(K)=i\left(\mathfrak{D}^{\prime}(R, K)\right)$. Replacing the block forms of $(21)$ in $\mathfrak{D}^{\prime}(R, K)$, we find

$$
\mathfrak{D}^{\prime}(R, K)=\left(\begin{array}{cccccccccc}
0 & 0 & 0 & 0 & 0 & I & 0 & B_{1} B_{0} & 0 & 0 \\
B_{\infty} B_{1} & 0 & 0 & 0 & 0 & 0 & 0 & 0 & 0 & I \\
0 & B_{\infty} B_{1} & 0 & 0 & B_{\infty} A_{1} & 0 & 0 & I & 0 & 0 \\
D_{\infty} B_{1} & 0 & I & 0 & 0 & 0 & 0 & 0 & 0 & 0 \\
0 & D_{\infty} B_{1} & 0 & I & D_{\infty} A_{1} & 0 & 0 & 0 & 0 & 0 \\
I & 0 & 0 & 0 & B_{0} B_{\infty} & 0 & 0 & 0 & 0 & B_{0} X \\
0 & 0 & 0 & 0 & 0 & B_{0} B_{\infty} & 0 & 0 & 0 & 0 \\
0 & 0 & 0 & 0 & 0 & 0 & I & D_{1} B_{0} & 0 & 0 \\
0 & 0 & 0 & 0 & D_{0} B_{\infty} & 0 & 0 & 0 & I & D_{0} X \\
0 & 0 & 0 & 0 & 0 & D_{0} B_{\infty} & 0 & 0 & 0 & I
\end{array}\right),
$$

where $A_{\bullet}=A_{\bullet}(K), B_{\bullet}=B_{\bullet}(K), C_{\bullet}=C_{\bullet}(K), D_{\bullet}=D_{\bullet}(K)$ and $X=X(K)$ for $\bullet \in\{0,1, \infty\}$. Doing a series of cancellations that correspond to the identity matrices which appear as the
$(1,6)$
$(3,8)$,
$(4,3)$,
$(5,4)$,
$(6,1)$,
$(8,7)$,
$(9,9)$ and
$(10,10)$ 
entries in the above block presentation, we obtain the equivalent matrix

$$
R_{r}(K):=\left(\begin{array}{cc}
0 & B_{0} X B_{\infty} \\
X B_{\infty} B_{1} & X B_{\infty} A_{1}+D_{0} X B_{\infty}
\end{array}\right) .
$$

Corollary 6.1 For a knot $K \subset Y$, let $Y(R, K)$ denote the three-manifold obtained by splicing the complement of $K$ and the complement of the trefoil. Then

$$
\widehat{\mathrm{HF}}(Y(R, K))=\imath\left(R_{r}(K)\right) \text {. }
$$

Proof The claim follows immediately from the above discussion.

For the trefoils, our computations imply that

$$
\begin{aligned}
X(R) B_{\infty}(R)=X(L) B_{\infty}(L)=0 & \Rightarrow R_{r}(R)=R_{r}(L)=0 \\
& \Rightarrow|\widehat{\operatorname{HF}}(Y(R, R))|=7,|\widehat{\mathrm{HF}}(Y(R, L))|=9 .
\end{aligned}
$$

The above computations agree with the computations of Hedden and Levine [5].

Corollary 6.2 For every knot $K$ in a homology sphere $Y$ we have

$$
\begin{aligned}
|\widehat{\mathrm{HF}}(Y(R, K))| & \geq\left(a_{0}(K)+a_{1}(K)+2 a_{\infty}(K)\right)-4 \min \left\{a_{0}(K), a_{1}(K), a_{\infty}(K)\right\} \\
& =4 \max \left\{h_{0}(K), h_{1}(K), h_{\infty}(K)\right\}-\left(h_{0}(K)+h_{1}(K)+2 h_{\infty}(K)\right) .
\end{aligned}
$$

Moreover, if $Y(R, K)$ is a homology sphere $L$-space, $K$ is trivial and $Y$ is a homology sphere $L$-space.

Proof Let $M=M(K)=X(K) B_{\infty}(K)$ and note that

$$
\begin{aligned}
\operatorname{rnk}\left(R_{r}(K)\right) & =\operatorname{rnk}\left(\begin{array}{cc}
0 & B_{0}(K) M \\
M B_{1}(K) & M A_{1}(K)+D_{0}(K) M
\end{array}\right) \\
& \leq \operatorname{rnk}\left(M B_{1}(K)\right. \\
& \left.M A_{1}(K)\right)+\operatorname{rnk}\left(\begin{array}{c}
B_{0}(K) M \\
D_{0}(K) M
\end{array}\right) \\
& =2 \operatorname{rnk}(M) \\
& \leq 2 \operatorname{rnk}(X(K)) .
\end{aligned}
$$

For every knot $K \subset Y$ as above note that the rank of $X=X(K)$ is at most equal to the minimum of the sizes of the matrices $B_{0}(K), B_{1}(K)$ and $B_{\infty}(K)$, which is

$$
\min \left\{a_{0}(K), a_{1}(K), a_{\infty}(K)\right\} .
$$

Since $R_{r}(K)$ is of size $h_{0}(K) \times h_{1}(K)=\left(a_{1}(K)+a_{\infty}(K)\right) \times\left(a_{0}(K)+a_{\infty}(K)\right)$, this proves the first part of the corollary. 
Let us assume that $\operatorname{rnk}(\widehat{\mathrm{HF}}(Y(R, K)))=1$. From here we find

$$
\begin{aligned}
\left(a_{0}(K)+a_{1}(K)+2 a_{\infty}(K)\right)-4 & \min \left\{a_{0}(K), a_{1}(K), a_{\infty}(K)\right\} \\
= & \left(a_{0}(K)+a_{1}(K)+2 a_{\infty}(K)\right)-4 \operatorname{rnk}(M)=1 .
\end{aligned}
$$

Since $a_{1}(K)$ and $a_{\infty}(K)$ have the same parity while the parity of $a_{0}(K)$ is different from the parity of both $a_{1}(K)$ and $a_{\infty}(K)$, one can easily conclude that $a_{0}(K)-1=$ $a_{1}(K)=a_{\infty}(K)$. Let $a$ denote the common value $a_{1}(K)=a_{\infty}(K)$. Then the rank of $M$ is $a$ and both $B_{0}(K)$ and $X(K)$ are invertible. We may thus assume that $A_{0}(K)=D_{0}(K)=0$. Since

$$
\operatorname{rnk}\left(\mathfrak{f}_{\infty}(K)+\overline{\mathfrak{f}}_{\infty}(K)\right)=\operatorname{rnk}\left(\begin{array}{cc}
B_{1}(K) A_{0}(K) & B_{1}(K) B_{0}(K) \\
I+D_{1}(K) A_{0}(K) & D_{1}(K) B_{0}(K)
\end{array}\right)=2 a,
$$

the three-manifold $Y$ is an $L$-space. Since splicing $K$ with the trefoil is also a homology sphere $L$-space, we conclude that $K$ is trivial, by [5, Theorem 1].

\section{Appendix: Bordered Floer homology for knot complements}

The first draft of this paper appeared while the theory of bordered Floer homology was being developed. With bordered Floer homology conventions widely known to the Heegaard Floer community, the referee recommended the inclusion of an appendix which addresses the contribution of this paper within the realm of bordered Floer homology.

Let $K \subset Y$ denote a null-homologous knot inside the three-manifold $Y$ and let $H=(\Sigma, \boldsymbol{\alpha}, \widehat{\boldsymbol{\beta}} \cup\{\lambda, \mu\} ; z)$ denote a special Heegaard diagram for $K$, as constructed in Lemma 4.1. In particular, $H$ is a nice Heegaard diagram for the bordered three-manifold $Y_{K}$ determined by $K \subset Y$ in the sense of Lipshitz, Ozsváth and Thurston [6]. The bordered Floer complex $\widehat{\mathrm{CFD}}\left(Y_{K}\right)$ may then be constructed from the chain complexes $M=M(K)$ and $L=L(K)$ (which are described in Proposition 5.1 as the mapping cones of $\overline{\mathfrak{f}}_{\infty}(K): C_{0}(K) \rightarrow C_{1}(K)$ and $\mathfrak{f}_{0}(K): C_{1}(K) \rightarrow C_{\infty}(K)$, respectively) and the chain maps $\Phi=\Phi(K): L \rightarrow M$ and $\Psi_{i}=\Psi_{i}(K): M \rightarrow L, i=1,2,3$.

More precisely and following the notation of [7, Section 4.2], the idempotents $l_{0}$ and $l_{1}$ and the chords $\rho_{1}, \rho_{2}, \rho_{3}, \rho_{12}=\rho_{1} \rho_{2}, \rho_{23}=\rho_{2} \rho_{3}$ and $\rho_{123}=\rho_{1} \rho_{2} \rho_{3}$ form an $\mathbb{F}$-basis for the differential graded algebra associated with the torus boundary:

$$
\mathcal{A}\left(T^{2}, 0\right)=\left\langle l_{0} \stackrel{\rho_{\rho_{3}}^{\rho_{2}}}{\rho_{\rho_{3}}} \cdot l_{1}\right\rangle /\left(\rho_{2} \rho_{1}=\rho_{3} \rho_{2}=0\right) .
$$


The module $\widehat{\mathrm{CFD}}\left(Y_{K}\right)$ is generated (over $\left.\mathcal{A}\left(T^{2}, 0\right)\right)$ by the generators of $M$ and $L$. For a generator $\boldsymbol{x}$ of $L$ we have

$$
I(\boldsymbol{x})=l_{0} \quad \text { and } \quad \partial(\boldsymbol{x})=d_{L}(\boldsymbol{x})+\rho_{1} \Psi_{1}(\boldsymbol{x})+\rho_{3} \Psi_{2}(\boldsymbol{x})+\rho_{123} \Psi_{3}(\boldsymbol{x}),
$$

while for a generator $\boldsymbol{y}$ of $M$ we have

$$
I(\boldsymbol{y})=l_{1} \quad \text { and } \quad \partial(\boldsymbol{y})=d_{\boldsymbol{M}}(\boldsymbol{y})+\rho_{2} \Phi(\boldsymbol{y}) .
$$

The splicing formula of (17) is then just the gluing formula for bordered Floer homology, ie [6, Theorem 1.3]. A related discussion is carried over in [6, Section 8].

Definition A.1 The chain complexes $\left(C_{\bullet}(K), d_{\bullet}\right), \bullet \in\{0,1, \infty\}$, and the chain maps $f_{\bullet}(K), \bar{f}_{\bullet}(K), \bullet \in\{0, \infty\}$, are called admissible data associated with the knot $K$ if they satisfy the following conditions:

- The homology of the complex $\left(C_{\bullet}(K), d_{\bullet}\right)$ is $\mathbb{H}_{\bullet}(K)$.

- The maps induced by $f_{\bullet}(K)$ and $\bar{f}_{\bullet}(K)$ in homology (under the identification of the homology of $\left(C_{\bullet}(K), d_{\bullet}\right)$ with $\left.\mathbb{H}_{\bullet}(K)\right)$ are $\mathfrak{f}_{\bullet}(K)$ and $\overline{\mathfrak{f}}_{\bullet}(K)$, respectively.

- We have $f_{0}(K) \circ f_{\infty}(K)=\bar{f}_{0}(K) \circ \bar{f}_{\infty}(K)=0$.

- The corresponding maps

$$
\begin{aligned}
& \theta(K): \operatorname{Ker}\left(\mathfrak{f}_{\infty}(K)\right) \longrightarrow \operatorname{Coker}\left(\mathfrak{f}_{0}(K)\right), \\
& \bar{\theta}(K): \operatorname{Ker}\left(\overline{\mathfrak{f}}_{\infty}(K)\right) \longrightarrow \operatorname{Coker}\left(\overline{\mathfrak{f}}_{0}(K)\right)
\end{aligned}
$$

are isomorphisms and are the inverses of the maps induced by $\mathfrak{f}_{1}(K)$ and $\overline{\mathfrak{f}}_{1}(K)$, respectively.

The proof of Theorem 1.1 implies that $\left(C_{\bullet}^{i}, d_{\bullet}^{i}\right)$ and the chain maps $f_{\bullet}^{i}, \bar{f}_{\bullet}^{i}$ for $\bullet \in\{0, \infty\}$ and $i=1,2$ in (17) may be replaced by other admissible data corresponding to the knots $K_{1}$ and $K_{2}$. orrespondingly, the bordered Floer complex associated with any knot $K \subset Y$ may be constructed from admissible data associated with $K$. More precisely, we have the following proposition:

Proposition A.2 Suppose that the chain complexes $\left(C_{\bullet}(K), d_{\bullet}\right), \bullet \in\{0,1, \infty\}$, and the chain maps $f_{\bullet}=f_{\bullet}(K), \bar{f}_{\bullet}=\bar{f}_{\bullet}(K), \bullet \in\{0, \infty\}$, are admissible data associated with the knot $K \subset Y$ and set

$$
M(K)=C_{0}(K) \oplus C_{1}(K), \quad L(K)=C_{1}(K) \oplus C_{\infty}(K) .
$$


The bordered Floer complex $\widehat{\mathrm{CFD}}\left(Y_{K}\right)$ may then be constructed as the left module over the differential graded algebra $\mathcal{A}\left(T^{2}, 0\right)$ which is generated by $\iota_{0} . L(K)$ and $\imath_{1} . M(K)$, and equipped with the differential $\partial: \widehat{\operatorname{CFD}}\left(Y_{K}\right) \rightarrow \widehat{\mathrm{CFD}}\left(Y_{K}\right)$ defined by

$$
\begin{aligned}
\partial\left(\begin{array}{l}
\boldsymbol{x} \\
\boldsymbol{y}
\end{array}\right) & \begin{cases}\left(\begin{array}{c}
d_{0}(\boldsymbol{x}) \\
\bar{f}_{\infty}(\boldsymbol{x})+d_{1}(\boldsymbol{y})
\end{array}\right)+\rho_{2} \cdot\left(\begin{array}{l}
0 \\
\boldsymbol{x}
\end{array}\right) & \text { if }\left(\begin{array}{l}
\boldsymbol{x} \\
\boldsymbol{y}
\end{array}\right) \in M(K), \\
\left(\begin{array}{c}
d_{1}(\boldsymbol{x}) \\
f_{0}(\boldsymbol{x})+d_{\infty}(\boldsymbol{y})
\end{array}\right)+\left(\begin{array}{c}
\rho_{1} f_{\infty}(\boldsymbol{x}) \\
\rho_{3} \bar{f}_{0}(\boldsymbol{y})+\rho_{1} \rho_{2} \rho_{3} \bar{f}_{0}\left(f_{\infty}(\boldsymbol{x})\right)
\end{array}\right) & \text { if }\left(\begin{array}{l}
\boldsymbol{x} \\
\boldsymbol{y}
\end{array}\right) \in L(K) .\end{cases}
\end{aligned}
$$

In particular, let the $\mathbb{F}$-modules $\mathbb{A}_{\bullet}=\mathbb{A}_{\bullet}(K), \bullet \in\{0,1, \infty\}$, and the matrices $A_{\bullet}=A_{\bullet}(K), B_{\bullet}=B_{\bullet}(K), C_{\bullet}=C_{\bullet}(K)$ and $D_{\bullet}=D_{\bullet}(K)$ be defined as in Section 3.3. Set

$$
\begin{gathered}
\left(C_{0}(K), d_{0}\right)=\left(\mathbb{A}_{\infty} \oplus \mathbb{A}_{1}, 0\right), \quad\left(C_{\infty}(K), d_{\infty}\right)=\left(\mathbb{A}_{1} \oplus \mathbb{A}_{0}, 0\right), \\
C_{1}(K)=\mathbb{A}_{1} \oplus \mathbb{A}_{0} \oplus \mathbb{A}_{\infty} \oplus \mathbb{A}_{1} \quad \text { and } \quad d_{1}=\left(\begin{array}{cccc}
0 & 0 & 0 & 0 \\
0 & 0 & 0 & 0 \\
0 & 0 & 0 & 0 \\
I_{\mathbb{A}_{1}} & 0 & 0 & 0
\end{array}\right) .
\end{gathered}
$$

Correspondingly, define

$$
f_{\infty}(K)=\left(\begin{array}{ll}
0 & 0 \\
0 & 0 \\
I & 0 \\
0 & I
\end{array}\right), \quad f_{0}(K)=\left(\begin{array}{cccc}
I & 0 & 0 & 0 \\
0 & I & 0 & 0
\end{array}\right) \quad \text { and } \quad \tau_{1}(K)=\left(\begin{array}{cccc}
0 & 0 & 0 & 0 \\
0 & A_{1} & B_{1} & 0 \\
0 & C_{1} & D_{1} & 0 \\
0 & 0 & 0 & 0
\end{array}\right)
$$

and set $\bar{f}_{\infty}(K)=\tau_{1}(K) f_{\infty}(K) \tau_{0}(K)$ and $\bar{f}_{0}(K)=\tau_{\infty}(K) f_{0}(K) \tau_{1}(K)$. The data associated with $K$ consisting of $\left(C_{\bullet}(K), d_{\bullet}\right)$ and $f_{\bullet}(K), \bar{f}_{\bullet}(K), \bullet \in\{0, \infty\}$ is then admissible.

Corresponding to the above admissible data and associated with $K \subset Y$, we may construct the bordered Floer complex for $K$ via

$$
\begin{gathered}
M(K)=C_{0}(K) \oplus C_{1}(K)=\mathbb{A}_{\infty} \oplus \mathbb{A}_{1} \oplus \mathbb{A}_{1} \oplus \mathbb{A}_{0} \oplus \mathbb{A}_{\infty} \oplus \mathbb{A}_{1}, \\
L(K)=C_{1}(K) \oplus C_{\infty}(K)=\mathbb{A}_{1} \oplus \mathbb{A}_{0} \oplus \mathbb{A}_{\infty} \oplus \mathbb{A}_{1} \oplus \mathbb{A}_{1} \oplus \mathbb{A}_{0}, \\
d_{M}=\left(\begin{array}{cccccc}
0 & 0 & 0 & 0 & 0 & 0 \\
0 & 0 & 0 & 0 & 0 & 0 \\
0 & 0 & 0 & 0 & 0 & 0 \\
B_{1} A_{0} & B_{1} B_{0} & 0 & 0 & 0 & 0 \\
D_{1} A_{0} & D_{1} B_{0} & I & 0 & 0 & 0 \\
0 & 0 & 0 & 0 & 0 & 0
\end{array}\right), \quad d_{L}=\left(\begin{array}{llllll}
0 & 0 & 0 & 0 & 0 & 0 \\
0 & 0 & 0 & 0 & 0 & 0 \\
0 & 0 & 0 & 0 & 0 & 0 \\
I & 0 & 0 & 0 & 0 & 0 \\
0 & 0 & I & 0 & 0 & 0 \\
0 & 0 & 0 & I & 0 & 0
\end{array}\right),
\end{gathered}
$$




$$
\Phi(K)=\left(\begin{array}{llllll}
0 & 0 & 0 & 0 & 0 & 0 \\
0 & 0 & 0 & 0 & 0 & 0 \\
I & 0 & 0 & 0 & 0 & 0 \\
0 & I & 0 & 0 & 0 & 0 \\
0 & 0 & I & 0 & 0 & 0 \\
0 & 0 & 0 & I & 0 & 0
\end{array}\right), \quad \Psi_{1}(K)=\left(\begin{array}{llllllll}
0 & 0 & 0 & 0 & 0 & 0 \\
0 & 0 & 0 & 0 & 0 & 0 \\
I & 0 & 0 & 0 & 0 & 0 \\
0 & I & 0 & 0 & 0 & 0 \\
0 & 0 & 0 & 0 & 0 & 0 \\
0 & 0 & 0 & 0 & 0 & 0
\end{array}\right)
$$

as the left module over the differential graded algebra $\mathcal{A}\left(T^{2}, 0\right)$ generated by $\imath_{0} . L$ and $\iota_{1} . M$ and equipped with the differential $\partial: \widehat{\mathrm{CFD}}\left(Y_{K}\right) \rightarrow \widehat{\mathrm{CFD}}\left(Y_{K}\right)$ defined by the equations (24) and (25).

Remark A.3 Simultaneous computation of the matrices $\tau_{\bullet}(K)=\left(\begin{array}{ll}A_{\bullet} & B_{\bullet} \\ C_{\bullet} & D_{\bullet}\end{array}\right)$ is a priori quite difficult, as we observed in the case of trefoils in Section 6. This makes the above description of the bordered Floer homology hard to use even for knots $K \subset Y$ where we have complete understanding of the Heegaard Floer complex associated with $K$. However, it is possible to construct admissible data associated with $K \subset Y$ completely in terms of the filtered chain complex $\mathrm{CF}^{\infty}(Y, K ; \mathbb{F})$, as will be discussed in the revision of [4].

\section{References}

[1] A Alishahi, E Eftekhary, A refinement of sutured Floer homology, J. Symplectic Geom. 13 (2015) 609-743

[2] E Eftekhary, Longitude Floer homology and the Whitehead double, Algebr. Geom. Topol. 5 (2005) 1389-1418 MR2171814

[3] E Eftekhary, A combinatorial approach to surgery formulas in Heegaard Floer homology, Algebr. Geom. Topol. 9 (2009) 2225-2246 MR2558310

[4] E Eftekhary, Bordered Floer homology and existence of incompressible tori in homology spheres, preprint (2015) arXiv:1504.05329

[5] M Hedden, A S Levine, Splicing knot complements and bordered Floer homology, preprint arXiv:1210.7055v1 To appear in J. Reine Angew. Math.

[6] R Lipshitz, O Ozsváth, D Thurston, Bordered Heegaard Floer homology: Invariance and pairing, preprint (2014) arXiv:0810.0687 
[7] R Lipshitz, O Ozsváth, D Thurston, Notes on bordered Floer homology, preprint (2014) arXiv:1211.6791

[8] C Manolescu, P Ozsváth, S Sarkar, A combinatorial description of knot Floer homology, Ann. of Math. 169 (2009) 633-660 MR2480614

[9] C Manolescu, P Ozsváth, Z Szabó, D Thurston, On combinatorial link Floer homology, Geom. Topol. 11 (2007) 2339-2412 MR2372850

[10] P Ozsváth, A I Stipsicz, Contact surgeries and the transverse invariant in knot Floer homology, J. Inst. Math. Jussieu 9 (2010) 601-632 MR2650809

[11] P Ozsváth, Z Szabó, Holomorphic disks and knot invariants, Adv. Math. 186 (2004) 58-116 MR2065507

[12] P Ozsváth, Z Szabó, Holomorphic disks and topological invariants for closed threemanifolds, Ann. of Math. 159 (2004) 1027-1158 MR2113019

[13] P Ozsváth, Z Szabó, On the Heegaard Floer homology of branched double-covers, Adv. Math. 194 (2005) 1-33 MR2141852

[14] PS Ozsváth, Z Szabó, Knot Floer homology and integer surgeries, Algebr. Geom. Topol. 8 (2008) 101-153 MR2377279

[15] S Sarkar, Maslov index formulas for Whitney n-gons, J. Symplectic Geom. 9 (2011) 251-270 MR2811652

[16] S Sarkar, J Wang, An algorithm for computing some Heegaard Floer homologies, Ann. of Math. 171 (2010) 1213-1236 MR2630063

School of Mathematics, Institute for Research in Fundamental Sciences (IPM) PO Box 19395-5746, Tehran 19395, Iran

eaman@ipm.ir

http://math.ipm.ir/ eftekhary

Received: 4 November 2013 Revised: 19 February 2015 
